\title{
Development of Novel Biodegradable Nanocomposites for Bone Repair Applications
}

\author{
By
}

\section{Samin Eftekhari}

\author{
Master of Science, Amirkabir Polytechnic University (2007) \\ Bachelor of Science, Sahand Polytechnic University (2003) \\ A dissertation presented to Ryerson University \\ in partial fulfillment of the \\ requirements for the degree of
}

Doctor of Philosophy

In

the Program of Chemical Engineering

Toronto, Ontario, Canada, 2016

CSamin Eftekhari 2016 


\section{AUTHOR'S DECLARATION}

I hereby declare that I am the sole author of this dissertation. This is a true copy of the dissertation, including any required final revisions, as accepted by my examiners.

I authorize Ryerson University to lend this dissertation to other institutions or individuals for the purpose of scholarly research.

I further authorize Ryerson University to reproduce this dissertation by photocopying or by other means, in total or in part, at the request of other institutions or individuals for the purpose of scholarly research.

I understand that my dissertation may be made electronically available to the public. 


\section{ABSTRACT \\ Development of Novel \\ Biodegradable Nanocomposites for Bone Repair \\ Applications}

\section{Samin Eftekhari}

Doctor of Philosophy, Chemical Engineering, Ryerson University (2016)

The main goal of this research is to introduce novel series of biodegradable nanocomposites that closely mimic the characteristics of real bone such as mechanical and thermal properties. These nanocomposites are composed of cotton-sourced cellulose microcrystals (MCC), hydroxyapatite nanoparticles (HA) and Poly L-Lactic Acid (PLLA). A novel fabrication route is used to manufacture MA and MH series of nanocomposites. MA series was developed to find an optimum range for weight fraction of each constituent required for design of the MH series. Evaluation of the thermal properties of MA series showed that increasing of weight ratio of MCC and HA from 0 to $21 \mathrm{~W}_{\mathrm{t}} \%$ increased the crystallinity up to $38 \%$. Compression test results of them revealed that increasing the weight fraction of MCC or HA from 0 to $21 \mathrm{~W}_{t} \%$ enhanced the compressive yield stress from 0.127 to $2.2 \mathrm{MPa}$ and the Young's modulus from 6.6 to 38 MPa. The cytotoxicity assay results showed there was no sign of toxic material affecting on viability of cells. The MH series was designed and fabricated by selecting a narrower range of weight fraction of the constituents. A design of experiments was used to alter the composition of the constituents to assess their contributions and their effect onto the mechanical properties and biodegradation behaviour of the $\mathrm{MH}$ series of the nanocomposites. The weight ratio of MCC to HA, the concentration of PLLA, and the 
porogen content were chosen as varying factors. A model that accurately predicts the optimum parameter setting was created. Analysis of variance statistical analysis showed that the ratio of MCC to HA was the most influential factor affecting the compressive yield and the mass loss, while the porogen content was the most detrimental factor affecting the Young's modulus of MH series of nanocomposites had no significant effect on their rate of the mass loss. The nanocomposites with highest weight ratio 4 of MCC to HA, showed maximum mechanical strength and the lowest water absorption and the lowest mass loss. It was found two series of nanocomposites was comparable to trabecular bone from a compositional, structural, thermal, mechanical point of view. 


\section{ACKNOWLEDGEMENTS}

I would like to acknowledge the Federal Government of Ontario (FEDEV) for providing Scientists and Engineers to Business fellowship award, the Office of Vice President for Research \& Innovation at Ryerson University for Science and Engineering for Business (SEB) fellowship award, the Norman Esch Foundation for Stage 1 and Stage 2 Norman Esch Engineering Innovation and Entrepreneurship awards, the Ontario Ministry of Research and Innovation for Early-Bird Award (MRI-ERA), and the Brookfield Partners Foundation for financially funding this project. Also, I would like to give my warm and sincere thanks to Dr. Habiba Bougherara, Dr. Jennifer Mactavish, Dr. Wendy Cukier, Jennifer MacInnis, Suraj Shah, and Dr. Rafik Loutfy for helping me to raise funding to financially support this project. Without their tremendous help and support, I would not have been able to reach to this point.

I would like to extend my most sincere thank to Dr. Habiba Bougherara who provided me with professional advice, an unconditional emotional support, constructive criticism, help, and support during my PhD journey and for her support in my new journey. Thank you Dr. Bougherara for being present during the toughest periods of my professional and personal life. I will never forget those days when you were on my side, while the ones who were expected to be present were absent.

I would like to give a very special and warm thanks to Dr. Ginette Turcotte for her unconditional support, advice, encouragement, and patience throughout this process. Also I would like to thank her for providing financial support for my international presentations. Thank you Dr. Turcotte for everything. 
I would like to give very special thanks to Dr. Ihab El Sawi for always being willing to provide me for those words of advice when I needed them the most. I do not have sufficient words to express how thankful I am for all your help, support, feedback, patience, and just being there for me.

I would like to extend my gratitude to Dr. Simant R. Upreti, Dr. Ramadhane Dhib, Dr. Huu Doan, Dr. Stephen D. Waldman, Dr. Derick Rousseau, Dr. Radovan Zdero, Dr. Sri Krishnan, Dr. Kaamran Raahemifar, Isabella Fernandes, Ali Hemmati, Tondar Tajrobehkar, Lynsey Kissane, Shelby Gaudrault, Michelle Colasuonno, Asma Shahsamand who helped me for my $\mathrm{PhD}$ research by providing in-kind support, professional advice, or constructive feedback.

I would like to extend my most sincere thanks to Dr. Rafik Loutfy, Charles Boulakia, and Dr. Erika Murray for their advice and help for patenting of this project.

I appreciate the work of my part-time employee and of my research assistants including Dr. Ihab El Sawi, Ali Motahhari, Hiba Gouse, Samar El Seyed, Alborz Soltankhah, and Negin Eftekhari for their contributions on running the experiments or proof-reading.

Also, I would like to acknowledge the in-kind support of Chemical Engineering Department, Mechanical \& Industrial Engineering Department, Chemistry Department, Aerospace Engineering Department, St. Michael's Hospital, and Center of Engineering Innovation \& Entrepreneurship for providing lab spaces and access to their facilities and machines to progress in this project. 


\section{DEDICATION}

I dedicate this work to my only sister, Negin who was present in my life under any circumstances, helping me through my challenges and cheering for my successes. 


\section{TABLE OF CONTENTS}

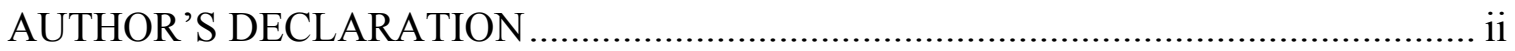

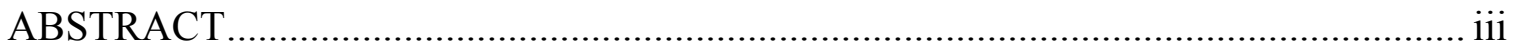

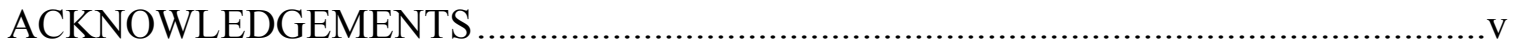

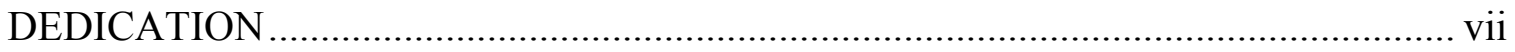

TABLE OF CONTENTS........................................................................................... vii

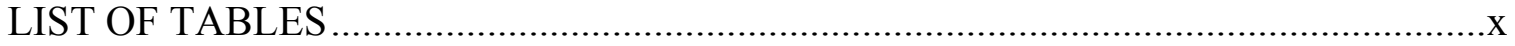

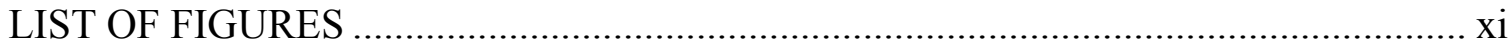

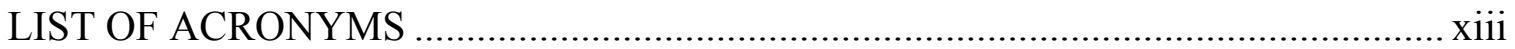

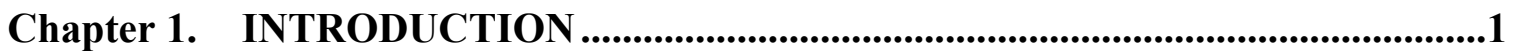

Chapter 2. LITERATURE REVIEW ...............................................................6

2.1. Bone type and structure ……………………….............................................

2.2. Required properties of orthopedic implants..........................................................

2.3. Composite and nanocomposite materials................................................................12

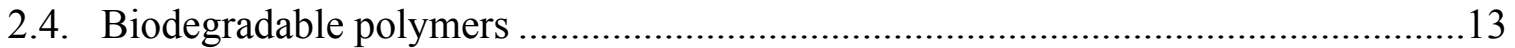

2.5. Treatment of nanosized reinforcing agents.......................................................... 18

2.6. Water absorption of the nanocomposites .......................................................19

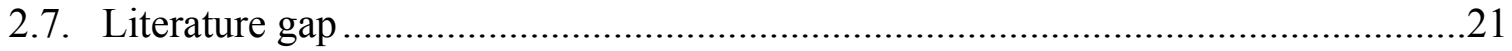

2.8. Research objectives.........................................................................................

Chapter 3. MATERIAL AND METHODS ...................................................................26

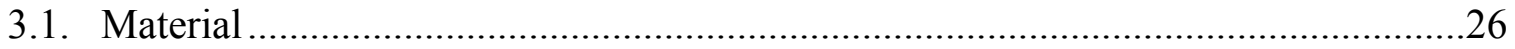

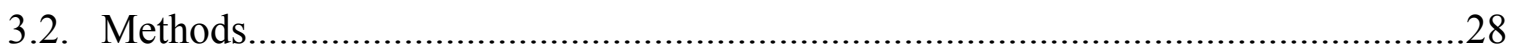

3.2.1. Fabrication procedure of MA series of nanocomposites ............................29

3.2.2. Fabrication procedure of MH series of nanocomposites ..............................31

3.2.3. Design of experiments of MH series of nanocomposites using general factorial design method .................................................................33

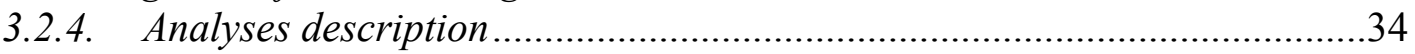

3.2.4.1. Fourier Transform Infrared Spectroscopy for chemical analysis ........................ 34

3.2.4.2. Differential Scanning Calorimetry (DSC) for thermal analysis ............................. 35

3.2.4.3. Scanning Electron Microscopy for morphological analysis ................................. 36

3.2.4.4. Liquid displacement method of the nanocomposites............................................. 37

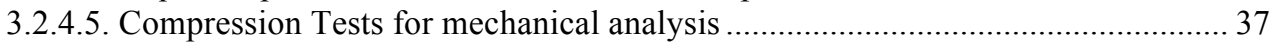

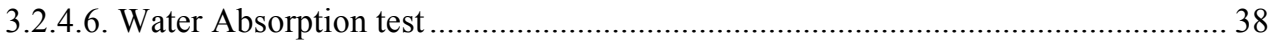

3.2.4.7. Mass Loss analysis for calculation of the degradation rate .................................. 38

3.2.4.8. MTT assay to evaluate the cytotoxicity of the nanocomposites ............................ 39

Chapter 4. RESULTS AND DISCUSSION.................................................................41

4.1. Physiochemical, thermal, and mechanical properties of the MA series of the nanocomposites 
4.1.1. Chemical analysis results of $M A$ series of nanocomposites .......................42

4.1.2. Crystallinity of the MA series of nanocomposites ....................................43

4.1.3. Compressive strength of the MA series of nanocomposites ........................47

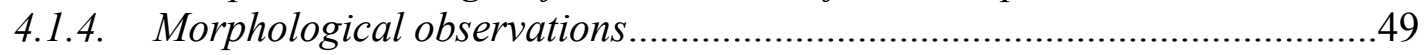

4.1.5. Toxicological studies of the MA series of nanocomposites .........................51

4.2. Optimization of the properties of the $\mathrm{MH}$ series of nanocomposites ......................52

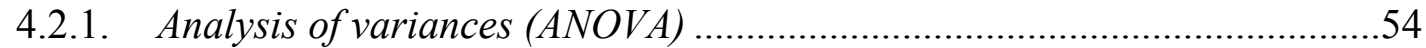

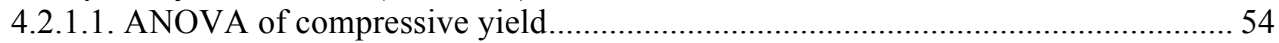

4.2.1.2. ANOVA of Young's modulus response .............................................................. 56

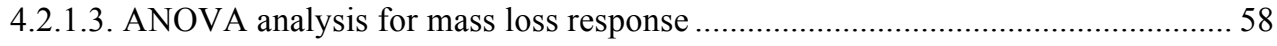

4.2.2. Optimization of conditions ........................................................................60

4.2.3. Influence of the porosity of the MH series of the nanocomposites onto the shape of their stress-strain curves

4.2.4. Investigation of the effect of the content of the cellulose and hydroxyapatite of the MH series of the nanocomposites onto the shape of the stress-strain curves

4.2.5. The effect of operating variables on the water absorption behaviour and mass loss of the of the MH series of nanocomposites

Chapter 5. CONCLUSIONS AND FUTURE WORK ........................................79

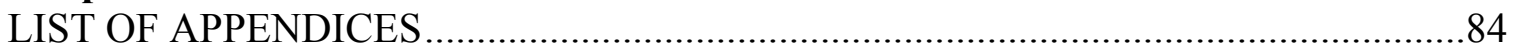

Appendix A. DSC graphs used for crystallinity calculation ...................................85

Appendix B. Image analysis results of MA series of nanocomposites.....................86

Appendix C. The picture of the microplates used for MTT toxicity assay ..............91

Appendix D. Final predictive equations in terms of coded factors.........................92

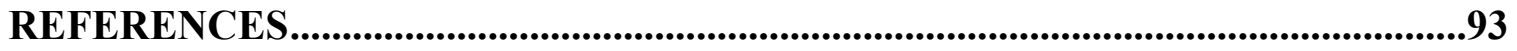




\section{LIST OF TABLES}

Table 1: Mechanical properties of cancellous (trabecular) bone of human body ...............8

Table 2: Mechanical properties of previously developed composites ..............................15

Table 3: Literature survey related to improve dispersibility of nano-phase agents ..........19

Table 4: Specimen nomenclature and composition of MA series of the

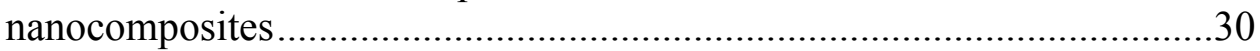

Table 5: Nomenclature of MH series of nanocomposite specimens without and

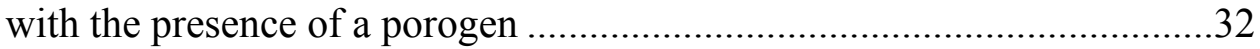

Table 6: Nomenclature of the MH series of the nanocomposites ..................................34

Table 7: Crystallinity of the raw PLLA, pristine sample, and MA series of nanocomposites ............................................................................45

Table 8: ANOVA table after model correction of compressive yield $\left(\sigma_{\mathrm{y}}\right)$ of the $\mathrm{MH}$ series of the nanocomposites

Table 9: The summary of the statistics for compressive yield of the of the $\mathrm{MH}$ series of nanocomposites

Table 10: Analysis of variance table for the Young's modulus of the MH series of

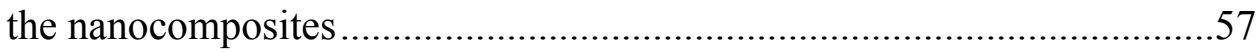

Table 11: The summary of the statistics for Young's modulus response

Table 12: ANOVA table for mass loss of the MH series of the MH series of nanocomposites.

Table 13: Summary of the statistics for the mass loss response 


\section{LIST OF FIGURES}

Figure 1: Composite structure of a bone (Morgan et al., 2005) ......................................

Figure 2: Scaffold guided bone tissue engineering (Ma \& Zhang, 2002) .......................11

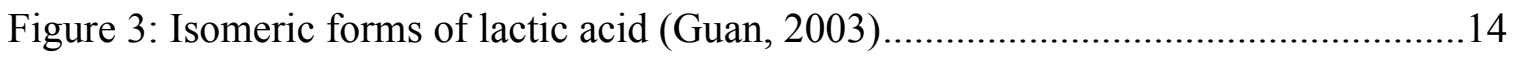

Figure 4: Structure of cellulose (Enchhorn et al., 2010)...............................................17

Figure 5: (a) Transparent PLLA solution in 1,4-Dioxane. (b) Homogenous colloidal dispersion of MCC in PLLA solution in the presence of SDS as coupling agent ...............................................................4 41

Figure 6: FTIR spectra of pristine specimen and of MA1010 nanocomposite .................43

Figure 7: DSC heating scans of the PLLA, pristine specimen, and the MA series

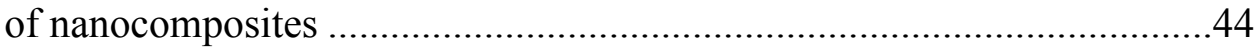

Figure 8: DSC heating and cooling curves of the MA5050 nanocomposite ....................46

Figure 9: Typical stress-strain curve of MA series of nanocomposites....

Figure 10: (a) Young's modulus (b) Compression yield of the MA series of nanocomposites

Figure 11: SEM micrographs of (a) Pristine, (b) MA3030, (c) MA5050, (d) MA6060 nanocomposites

Figure 12: SEM micrographs illustrating a representative cross-section of MA4040 nanocomposite (a) raw micrograph (b) the estimated porous areas

Figure 13: Cells viability of rat cells after incubation in extracts of MA4040, MA5050, and pristine nanocomposites for 24, 48, and 72h. Control group without extract

Figure 14: The desirability values for optimization of the parameters to obtain maximum compressive properties and minimum mass loss of the $\mathrm{MH}$ series of the nanocomposites

Figure 15: The compressive yield values for optimization of the parameters to obtain maximum compressive properties and minimum mass loss of the $\mathrm{MH}$ series of the nanocomposites.

Figure 16: The Young's modulus values for optimization of the parameters to obtain maximum compressive properties and minimum mass loss of the $\mathrm{MH}$ series of the nanocomposites....

Figure 17: The mass loss values for optimization of the parameters to obtain maximum compressive properties and minimum mass loss of the $\mathrm{MH}$ series of the nanocomposites 
Figure 18: Typical stress-strain curves of the nanocomposites with identical ratio of $\mathrm{MCC} / \mathrm{HA}=4$ and various concentrations of PLLA and porogen content .66

Figure 19: SEM images of nanocomposites, (a) MH 204 N, (b) MH $204 \mathrm{P}$

Figure 20: Typical stress-strain curve for the MH series of the nanocomposites reinforced by HA nanoparticles solely

Figure 21: SEM images of nanocomposites MH 204 P, (c) MH 100 N, (d) MH $150 \mathrm{~N}$, (e) MH $200 \mathrm{~N}$

Figure 22: Compressive yield versus ratio of the $\mathrm{MCC} / \mathrm{HA}$ of the $\mathrm{MH}$ series of the nanocomposites with concentration of PLLA $(A=20)$, zero porogen content $(\mathrm{C}=0)$

Figure 23: Water absorption curves of three $\mathrm{MH}$ series of the nanocomposites varying MCC to HA ratios, green curve, red curve, and the blue curve have the ratio of MCC to HA equal to $0,1,4$, respectively, the concentration of PLLA equal to $20 \%$ and porogen content equal to 0 for all three nanocomposites

Figure 24: The effect of ratio of MCC to HA (factor B) onto water absorption of the (MH $200 \mathrm{~N}),(\mathrm{MH} 201 \mathrm{~N})$, and (MH $204 \mathrm{~N})$ nanocomposites which have various ratio of $\mathrm{MCC} / \mathrm{HA}$ equal to 0,1 , and 4 , but identical concentration of PLLA equal to 20 (factor A) and porogen content (factor $\mathrm{C}$ ) equal to 0 .78 


\section{LIST OF ACRONYMS}

Term

Analysis of variances

Design of experiments

HA

MCC

PLLA

PBS

3 dimensional

TCP
Initial components of the term

ANOVA

DOE

Hydroxyapatite

Micro crystalline cellulose

Poly (L-lactic) acid

Phosphate buffer saline

3D

Tissue culture plastic 


\section{Chapter 1. INTRODUCTION}

Bone fractures are one of the most common forms of bone injuries. Traffic collisions, injuries, diseases, maxillofacial defects or bone tumors cause many bone fractures, so all above require some kind of orthopedic interventions to help the damaged bone to heal (Blitterswijk et al. 2008). Approximately 900,000 surgeries requiring bone grafting are performed annually in US alone, according to the U.S. Centers for Medicare and Medicaid Services. One of the common treatments performed by orthopedic surgeons is bone grafting. These grafts are taken from the patient's own body (autografts) or from an external source such as other individuals (allografts). Autografts can cause complications such as chronic pain at donor site and morbidity, and allografts have a considerable chance of being rejected by the patient's body and a risk of transmission of diseases. Accordingly, the lack of bone graft supply has raised the need for other alternatives for human bone products. Metallic orthopedic implants are being used as permanent implants for replacement of missing or damaged bone. Their intrinsic properties such as stiffness make them suitable candidate for load-bearing applications. However, mismatch of the mechanical properties and density of metallic implant with the surrounding bone may cause stress shielding which results in loosening of the implant. Besides, mismatch of the thermal properties and difference in density of the metallic implant with the surrounding bone may cause discomfort at implantation site for the patients in cold weather. So, metallic implants must be extracted from the body after bone repair process is completed. These revision surgeries not only increase the number of admissions to the hospitals, but also cause additional costs to the healthcare industry and insurers. So far, an appropriate 
synthetic bone graft to replace healthy bone that has all the advantages of the patient's own bone without its disadvantages does not exist. Bone has some unique properties like being porous and having mechanical strength. These properties can be related to its complex nanocomposite structure in which collagen fibers wrapped with calcium phosphate minerals are embedded in a matrix (Ratner et al. 2004). Bioresorbable orthopaedic implants such as fixation instruments may provide a unique and promising solution to unmet clinical needs. Since the mechanical properties of human bone vary tremendously according to the location of the bone and function of it in the body (i.e. load bearing or non-load bearing), the mechanical properties of the synthetic bone grafts or substitutes must be tailored in order to match the properties of the surrounding bone at the damaged site (Amini et al. 2012).

Prior synthetic bone grafts composed of ceramic/polymer nanocomposites mimicking certain properties of bone have been used with limited success. Since polymers can represent matrix of the bone successfully. For instance, Poly L- Lactic Acid (PLLA) is a biocompatible and biodegradable polymer that has been widely used in biomedical applications. However, PLLA-based composites have had some drawbacks such as low mechanical properties and acidic degradation by-products (Nejati et al. 2008). Hydroxyapatite (HA) has many similarities with the calcium phosphates of real bone, and has been used in biomimetic applications. Although HA with certain, limited, functionality have been suggested for use as bone scaffolds or grafts, many crucial challenges remain unsolved. For example, one of the ongoing challenges is increasing the mechanical strength of the composites made up of PLLA and HA without sacrificing their elongation at break or decreasing their absorbed energy before break (ductility). 
Previously developed PLLA/HA composites crack under stress due to the brittle nature of HA (Masami et al. 2013; Roether et al. 2002; Pei et al. 2010). Based on literature review (Pooyan et al. 2012) and taking the inspiration from natural composition of real bone, utilization of the flexible biopolymers as a second reinforcing agent, were introduced to address these problems. Natural biopolymers including cellulose not only possess mechanical strength but they also present many advantages over other synthetic reinforcing agents like being flexible, low density, low cost, and being bioabsorbable (Sturcova et al. 2005; Eichhorn et al. 2010; Armentano et al. 2013). However, the hydrophilic nature of natural fibers creates two main challenges for composites or nanocomposites that contain them:

(1) The first challenge is low compatibility of natural fibers with hydrophobic polymeric matrices due to their hydrophilic nature, which causes dispersion difficulties in an organic solvent and poor interfacial bonding between the polymeric matrix and the reinforcing agents. This will deteriorate the final properties of the nanocomposites ultimately such as mechanical properties. So the first stage of this research focused on development and fabrication of the MA series of nanocomposites consisting of PLLA reinforced with cottonsourced microcrystalline cellulose (MCC) and HA nanoparticles. The dispersion difficulty of the hydrophilic MCC and HA nanoparticles in a hydrophobic PLLA solution was overcome by utilizing a coupling agent and a unique fabrication method. Then the properties of the MA series of the nanocomposites were evaluated to move the research to the second stage. In the second stage of this study, the MH series of the nanocomposites were 
designed by using the preliminary results obtained from characterization of the MA series of the nanocomposites. Therefore, the MH series of the nanocomposites designed to improve the properties of the MA series of the nanocomposites such as mechanical properties and to control the water absorption and the biodegradation rate of the nanocomposites.

(2) The second challenge in terms of utilization of natural fibers is the high in affinity towards water absorption. Biomaterials containing cellulose absorb water rapidly which may result in sooner than expected biodegradation, because faster water absorption accelerates the biodegradation of the PLLA polymers due to hydrolysis (Goetz \& Oksman, 2009). Also, this phenomenon would cause undesired and catastrophic changes in vivo due to swelling and changing the size of the implant and deterioration of the mechanical strength. So, improving the water absorption resistance and decreasing the biodegradation rate of cellulose-containing biomaterials without sacrificing their mechanical properties especially with higher contents of natural fibers is the main goal of this stage of this study. Since the composition of the nanocomposite materials affects their properties, an attempt was made to alter systematically the composition and porosity of the MH series of the nanocomposites to assess the contribution of composition and porosity to their mechanical properties, water absorption and biodegradation. In this stage of the study the simultaneous influence of three independent factors (variables), including the concentration of PLLA, the ratio of the cellulose over hydroxyapatite, and the porogen content, on final properties were investigated. 
Since, tailoring the mechanical behaviour and biodegradation rate of the nanocomposite biomaterials is possible by effectively controlling the weight fraction, ratio of the reinforcing agents (MCC and HA) and their type. Then, an analysis of variances (ANOVA) was used in order to identify and quantify the contribution of each factor affecting the final properties. The optimization of the conditions to maximize the mechanical properties and minimize the water absorption amount or the mass loss of the MH series of nanocomposites projected as bone grafts or scaffolds was the final stage of this project. Finally, the influence of the porosity of the nanocomposite and its effect on the stress-strain behaviour of the $\mathrm{MH}$ series of the nanocomposite was evaluated in detail as well. So, after all these analyses, the biodegradation rate of the $\mathrm{MH}$ series of the nanocomposites as synthetic bone grafts can be controlled based on the patient's age to match the rate of the bone regeneration process. 


\section{Chapter 2. LITERATURE REVIEW}

The second most common transplantable tissue after blood is bone (Chu \& Xuanyong, 2008). The global bone graft substitute market was valued at $\$ 1.9$ billion (US) in 2010 according to "companiesandmarkets.com", and increased to \$2.1 billion in 2013 (globaldata.com). The market is forecasted to reach $\$ 2.6$ billion in 2020, with compound annual growth rate of $3.5 \%$. The total Canadian market size for demineralized bone products were between US $\$ 13$ to $\$ 18$ million in 2005-2006. Traffic collisions, injuries, diseases, maxillofacial defects, and bone cancers or tumors are causing these injuries and bone losses that need some kind of intervention. Furthermore, by 2040, the senior citizen population will double in the U.S. alone to about 70 million and consequently the cost of healthcare will increase drastically by that time (Medipoint, 2014).

When the self-healing mechanism of bone fails as a result of defect size, infection or other causes, bone grafting is needed. Bone grafting means that bone from somewhere else is applied to the damaged site in order to fill the gap and stimulate bone formation. Preferably, it is the patient's own bone (autograft) that is harvested from locations of relative excess of bone (called the donor-site), such as the pelvis. Bone of other individuals (allograft) or animals (xenograft) can be used as well. But there are lots of serious problems are associated with their application for bone grafting. For instance, the usage of autografts is limited by the increased possibilities of morbidity, chronic pain at the donor-site, and lack of bone supply. On the other hand, allografts and xenografts have constraints such as donor-site rejection, as well as increased chances of disease transmissions. 
Metals such as pure or in alloyed forms of titanium are being used as orthopaedic implants. They have some excellent properties such as stiffness, which meet the necessary requirements for load-bearing bones. However, metallic implants may fail due to mismatch of the mechanical and thermal properties with real bone. The difference between strength of the metallic implant and strength of the surrounding bone may cause stress-shielding phenomena that may loosen the implant. Besides, mismatch of thermal properties of the metallic implant and the surrounding bone may cause pain at implanted site for the patients in cold weather. So, in case of fracture fixation instruments, the permanent metallic implants are better to be extracted from body, which will force extra cost of the revision surgery for the hospitals and inconvenience to the patients and surgeons.

Yet, bioresorbable implants may provide a promising solution for these clinical problems. So far, an appropriate alternative for replacing injured or missing bone that has all the advantages of autograft bone without its disadvantages does not exist. Therefore, in order to fabricate novel orthopaedic implants that successfully resemble real bone in terms of chemical, thermal, morphological, and mechanical properties, the type of the bones in human body, its constituents, and mechanical properties are studied in following section.

\subsection{Bone type and structure}

There are two types of bone in human skeleton. Compact bone, also called cortical bone, is a dense bone in which a bony matrix is filled with organic and inorganic compounds, leaving tiny spaces that contains bone cells. It makes up to 80 percent of the human skeleton, where the greater strength and rigidity is needed; and cancellous bone, also 
called trabecular bone, which is more porous like a sponge and has less strong properties in comparison with compact bone (Martens et al. 1983). The mechanical properties of human cancellous (trabecular) bone are shown in Table 1.

Table 1: Mechanical properties of cancellous (trabecular) bone of human body

\begin{tabular}{|cccc|}
\hline $\begin{array}{c}\text { Type of bone } \\
\begin{array}{c}\text { Cancellous } \\
\text { bone }\end{array}\end{array}$ & $\begin{array}{c}\text { Compressive yield } \\
(\mathrm{MPa})\end{array}$ & $\begin{array}{c}\text { Young's modulus } \\
(\mathrm{MPa})\end{array}$ & References \\
\hline
\end{tabular}

Bone is a dynamic tissue with a unique capability to heal and remodel by itself. It possesses a unique combination of properties such as high compressive and tensile strength despite its highly porous microstructure. The exceptional chemical composition of bone consists of organic (collagen) and inorganic (minerals) constituents that give bone these properties. Inorganic constituents such as calcium phosphate minerals, give bone its mechanical properties, such as stiffness, strength, hardness, and resilience. The organic constituents such as collagen give bone its viscoelastic properties. As shown in Figure 1, a real bone is a biological composite material in which collagen fibers strongly bonded and wrapped with calcium phosphate minerals and embedded in a matrix.

Since bone itself is a true nanocomposite, the combination of natural biopolymers and synthetic materials has been utilized in order to introduce novel nanocomposites to mimic compositional and chemical characteristics of real bone. Additionally, bone has a threedimensional (3D) porous structure. Various artificial bone substitute constructs have been developed to mimic structural features of real bone to meet the required mechanical strength. So far, none of the existing biomaterials possess all the required properties for various orthopaedic applications to be used in different locations of the body. Therefore, 
the ongoing challenge is to find suitable materials with desired properties to mimic this complicated organ when bone replacement is needed.

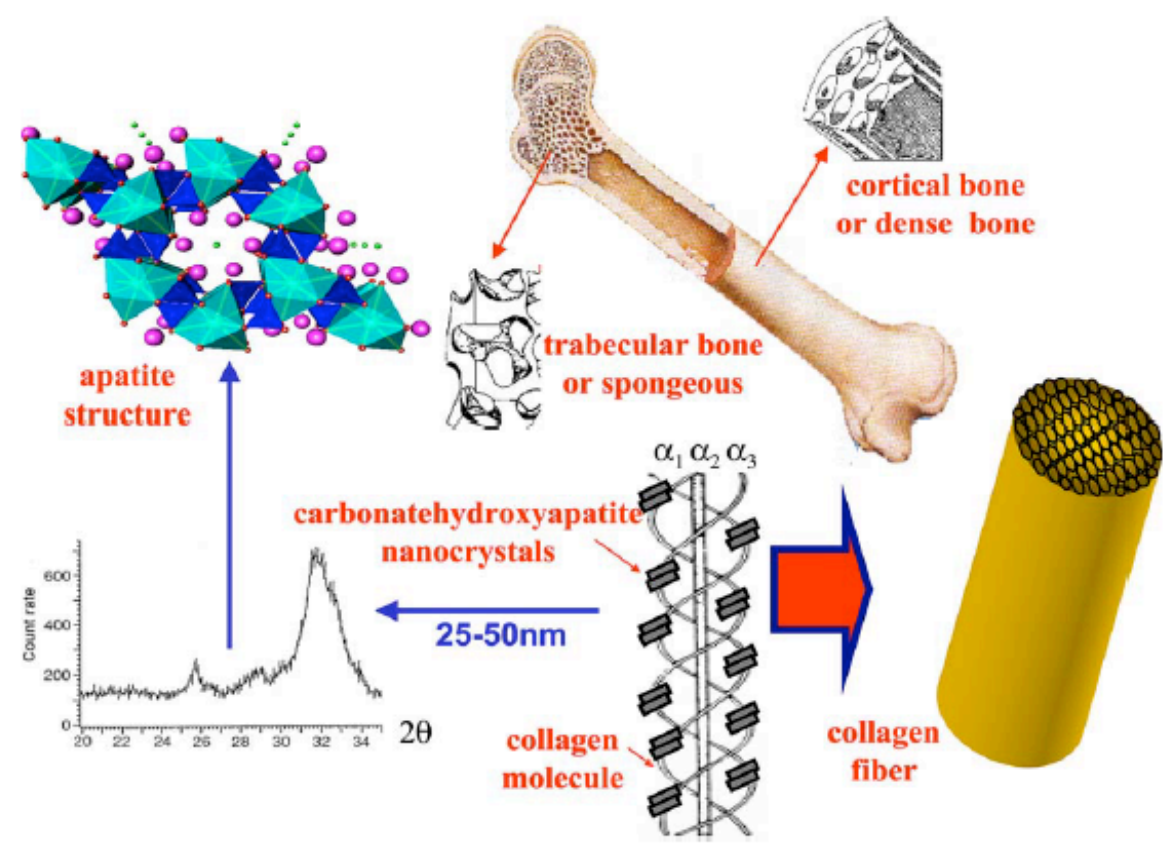

Figure 1: Composite structure of a bone (Morgan et al., 2005)

\subsection{Required properties of orthopedic implants}

An orthopedic implant is a medical device designed to support a damaged or fractured bone, and in some cases to replace missing bone. This definition encompasses all the medical devices such as artificial bone substitutes, synthetic bone grafts, bone scaffolds, and fracture fixation instruments such as plates, and screws. All orthopedic implants should possess the required properties based on the proposed application of the implant or the location of the damaged bone to assist healing process. These properties are as follows: 
Biocompatibility is perhaps the most important requirement in orthopaedic implants. The implanted biomaterial should not initiate any inflammatory or toxic response that will result in bone cell death.

Mechanical properties are those that involve a relationship between stress and strain to an applied physical force. A bone graft or a bone scaffold needs to be strong enough to maintain its shape while it is under stress.

Chemical properties of an implant are important because they will define the final mechanical, biological, and thermal properties of the final implant. Furthermore, the surface functional groups of the implant can influence the interaction of the biomaterial with surrounding tissue, which will determine the final success of the implant.

Biodegradability means the body can eat up the biomaterial after repair is completed. When the orthopaedic implant such as bioabsorbable bone scaffolds and fixation instruments are being used for non-permanent bone repair applications, biodegradable bone substitutes can be beneficial. The advantage is two-fold: the supportive construction does not hinder the repair process of new bone and any detrimental long-term tissue reactions of the body are prevented. Besides, the construct degrades at a rate that matches the formation of new bone tissue. Non-degradable metallic screws used for fracture fixation are causing some problems such as interrupting growing of bone tissue around the implant, infection, and pain for the patient. So, removal of them is required after certain amount of time by performing a second surgery. Therefore, biodegradable fixation screws could be the appropriate alternative. As exhibited in Figure 2, scaffolds provide structural support for bone-generating cells to adhere, grow and regenerate new bone, and then biodegrade in live body environment. They repair or regenerate damaged 
tissue by using engineered substitutes called scaffolds. Functionality can be maintained during the healing and finally can be combined with the host tissue.

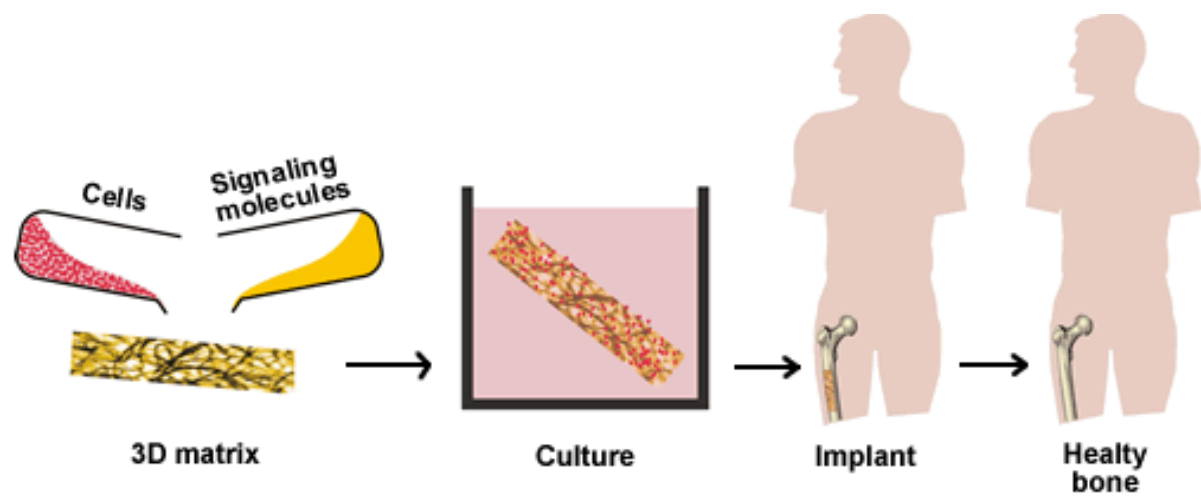

Figure 2: Scaffold guided bone tissue engineering (Ma \& Zhang, 2002)

Surface properties influence the initial cell interactions with the biomaterial and involve physicochemical properties such as surface hydrophobicity, heterogeneity, topography, roughness and functional groups.

Topographical properties are of particular interest when the topic is osteoconduction (ability to bond and integrate with bone). Biological evidence prove that rough surfaces will have better osteoconductive properties than smooth surfaces, and will facilitate the migration of osteogenic cells to the materials surface (Davies, 1980).

Porosity is also important in case of bone scaffolds for diffusion of nutrients or gases and for the removal of metabolic waste resulting from the activity of the cells growing into the scaffold. However, the degree of porosity always influences other properties of the scaffolds such as its mechanical stability. So this value should always be balanced with the mechanical requirements of the targeted bone, which is going to be replaced. Yang and Leong (2001) concluded that the appropriate porosity percentage for efficient bone scaffolds is over $75 \%$ volume. Acceptable range for pore size is between $200-900 \mu \mathrm{m}$ for 
bone scaffold applications (Wei \& Ma., 2004; Agrawal \& Ray, 2001), however, the other resources reported the acceptable pore size range for this application 75-250 $\mu \mathrm{m}$ (Ratner, 2004). There is still research going on selecting a novel combination of materials to mimic real bone in terms of physiochemical, mechanical, thermal, and biological properties. Nanocomposite biomaterials can represent bone tissue very well, since bone itself is a true nanocomposite. So, it is beneficial to learn more about the composite and nanocomposite structures and the challenges for the fabrication of them.

\subsection{Composite and nanocomposite materials}

The word composite means "consisting of two or more distinct parts". For the purpose of this research, composites can be considered materials consisting of two or more chemically distinct constituents, having distinct interface separating them from each other (Ratner et al. 2002). Composites usually consist of one or more discontinuous phases embedded within a continuous phase. The discontinuous phase, which is usually harder and stronger than a continuous phase, is called the reinforcement or reinforcing material, whereas the continuous phase is called the matrix. If the reinforcing agent is in the nanoscale, the resulting composite is a nanocomposite. Properties of composites are strongly influenced by the properties of their constituents, their size, their distribution, the chemical interaction and bonding between them. Therefore, in describing composite materials, besides specifying constituent of the materials and their properties, geometry of the reinforcement agents, their distribution, and their concentration in the composite are key factors that determine the ultimate properties of the nanocomposites. Most composite materials are fabricated to have improved mechanical properties such as 
strength, stiffness, toughness, and fatigue resistance for projected applications. The strengthening mechanism strongly depends on the geometry and concentration of the reinforcement agents. Bone is certainly a nanocomposite with a number of levels of hierarchy at the molecular and microstructural level. Its properties are highly anisotropic, so the only possibility to mimic real bone is utilization of nanocomposites. Therefore, polymeric based nanocomposites in combination with different materials and reinforcing agents enables the design of the bone substitutes, grafts, and scaffolds with specific mechanical and biological properties and degradation rates. Design flexibility, strength, and low weight have made polymeric-based composite materials ideal materials for bone repair applications (Ma \& Zhang, 2002).

\subsection{Biodegradable polymers}

Synthetic polymers can be synthesized reproducibly and processed easily into variable shapes and sizes. Usually polymers are being used as a structural host matrix for nanoparticles or nanofibres. Poly Lactic Acid (PLA), poly glycolic acid (PGA) and their co-polymers are recognized biocompatible polymers that have been used for manufacturing of orthopaedic implants due to their relative high stiffness, biodegradability as well as biocompatibility. PLA is a type of biodegradable poly ( $\alpha$ ester). Lactic acid is found in blood and muscle tissue. As indicated in Figure 3, PLA has two isomeric forms, D (-), and L (+). Poly L-Lactide Acid (PLLA) can occur in crystalline forms as high as 37\%. Higher crystallinity results in higher mechanical properties and slower degradation. These characteristics make them suitable for their utilization in fabrication of bone substitutes, orthopaedic implants, and bone scaffolds. 


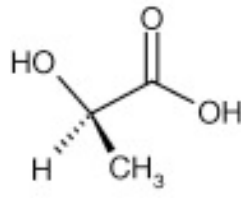

L - Lactic acid

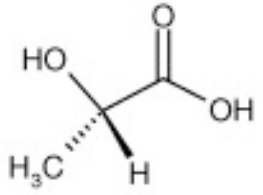

D - Lactic acid

Figure 3: Isomeric forms of lactic acid (Guan, 2003)

PLA isomers degrade via hydrolysis of ester chain bonds in vivo and their degradation products (lactic- and glycolic acid) are part of the Krebs metabolic cycle. However, physical, thermal, and mechanical properties of the PLLA are still not adequate enough for load-bearing applications (Gay et al. 2009). On the other hand, acidic biodegradation by-products of PLLA decrease the $\mathrm{pH}$ of implantation site in vivo, which may cause necrosis of the surrounding tissue (Xiao \& Zhang, 2007). The best approach is designing polymeric based composite or nanocomposite materials and reinforcing them with harder and stronger constituents such as nano-fibers, micro or nano crystals or nano particles. One common approach is to strengthen the polymeric matrix by utilization of reinforcing agents such as hydroxyapatite nanoparticles (HA) for $\backslash$ bone repair purposes.

HA is the most important bioceramic due to its bioactivity and chemistry similarity to natural minerals of bone. Table 2 shows the literature review about the prior art composites and nanocomposites developed to meet the expectation in the field of orthopaedic applications. HA is not thermodynamically stable at physiological $\mathrm{pH}(7.4)$ but it encourages bone-bonding process. The use of synthetic HA has shown positive effect on buffering the acidic environment of the implantation site of the nanocomposite (Ciobanu et al., 2009). 
Table 2: Mechanical properties of previously developed composites

\begin{tabular}{|c|c|c|c|c|}
\hline \multirow[b]{2}{*}{$\begin{array}{l}\text { Biodegradable } \\
\text { Polymers }\end{array}$} & \multirow{2}{*}{$\begin{array}{c}\begin{array}{c}\text { Reinforcing } \\
\text { agent }\end{array} \\
\begin{array}{c}\text { Volume } \\
(\%)\end{array}\end{array}$} & \multicolumn{2}{|r|}{ Composite } & \multirow[b]{2}{*}{ References } \\
\hline & & $\begin{array}{l}\text { Porosity } \\
(\%)\end{array}$ & $\begin{array}{c}\text { Mechanical Properties } \\
\text { (MPa) }\end{array}$ & \\
\hline PLLA-PLGA (75/25) & HA $(10-70)$ & 85 & $\begin{array}{l}\text { Young's modulus } 10-14 \\
\text { Compressive strength } 0.4\end{array}$ & $\begin{array}{l}\text { (Xiao et al. } \\
\text { 2007) }\end{array}$ \\
\hline PCL/PLGA (65/35) & HA $(0-50)$ & 80 & $\begin{array}{l}\text { Young's modulus } 12.5 \\
\text { Compressive strength } 0.51\end{array}$ & $\begin{array}{l}\text { (Guan, } \\
\text { 2003) }\end{array}$ \\
\hline PLLA & 0 & 93 & $\begin{array}{l}\text { Young's modulus } 6.4 \\
\text { Compressive Strength } 0.32\end{array}$ & $\begin{array}{c}\text { (Ma \& } \\
\text { Zang, 2002) }\end{array}$ \\
\hline PLLA & HA (50) & 80 & $\begin{array}{l}\text { Young's modulus } 10.9 \\
\text { Compressive strength } 0.39\end{array}$ & $\begin{array}{c}\text { (Ma } \\
\text { \&Zhang, } \\
\text { 2002) }\end{array}$ \\
\hline PLLA/PLGA & $\begin{array}{l}\text { Bioactive } \\
\text { glass }\end{array}$ & 43 & $\begin{array}{l}\text { Young's modulus } 51 \\
\text { Compressive strength } 0.42\end{array}$ & $\begin{array}{l}\text { (Mattila, } \\
\text { 2004) }\end{array}$ \\
\hline Porous HA & 0 & 82 & $\begin{array}{l}\text { Young's modulus } 0.83 \times 10^{-3} \\
\text { Compressive strength } 0.2-0.4\end{array}$ & $\begin{array}{l}\text { (Nejati et al. } \\
\text { 2009) }\end{array}$ \\
\hline PLLA/Flax nano fibers & $5 w t \%$ & - & Tensile strength 24.3 & $\begin{array}{c}\text { (Liu \& } \\
\text { Easteal, } \\
\text { 2010) }\end{array}$ \\
\hline
\end{tabular}

There is evidence that HA can encourage osteo-conductivity of bone substitutes (Wang, 2009). But HA particles do not make strong bonds easily at interface with the polymeric matrix like PLLA due to the existence of non-polar groups in the chain of PLLA (Ciobanu et al., 2009). According to Ciobanu, non-polar polymers such as PLLA have almost no affinity to polar reinforcing agents such as HA. 
In many cases, failure in composites is caused by the weak interface bonding between the reinforcing agent and the matrix. So improving the interface bonding of HA with matrix is still an ongoing challenge in preparation of HA-containing polymeric composites and nanocomposites. The reinforcing power of HA nanoparticles is not adequate for loadbearing applications.

Also, because of the brittle nature of HA, elongation at break of nanocomposites containing HA may decreae. So, biomaterials containing HA would crack under stress and this limits the use of HA in load-bearing applications. For instance, Guan (2003) fabricated PLGA based composites and reinforced them with HA particles. Although, he improved the mechanical properties of the composites in comparison with PLGA specimens, the load-bearing property of his PLGA-HA composites were not high enough to be used as bone graft substitutes. Weak interfacial bonding of PLLA-HA composites was always a challenge for researchers who deal with them since it affects inversely the reinforcing efficiency of the HA particles (Nejati et al., 2009).

The incorporation of more flexible reinforcing agents such as cellulose fibers or crystals will reduce the composite brittleness (Wang, 2009). Plant source fibers such as cotton source cellulose may be seen as a potential source of natural based biopolymers as reinforcing agents due to their renewability, low density, high specific strength and modulus, and high aspect ratio (length/diameter). Cellulose is an appropriate reinforcing constituent with relative high strength and stiffness due to its extended chain structure (Bondeson et al., 2006). Besides, cellulose crystals bonded to hydroxyapatite 
nanoparticles can resemble collagen fibrils existing in the real bone in which collagen fibers wrapped with calcium phosphate minerals (Ratner et al., 2002).

As shown in Figure 4, cellulose is a linear polysaccharide, which consists of hydroxyl functional groups, up to $15,000 \mathrm{D}$-glucose residues by $\beta(1 \Rightarrow 4)$ - glycosidic bonds (Voet, 1995). All these hydroxyl groups make it hydrophilic and susceptible to absorb water. Besides, presence of hydroxyl functional groups makes it suitable substance to immobilize biomolecules such as bone morphogenic proteins (BMP) to encourage new bone formation.

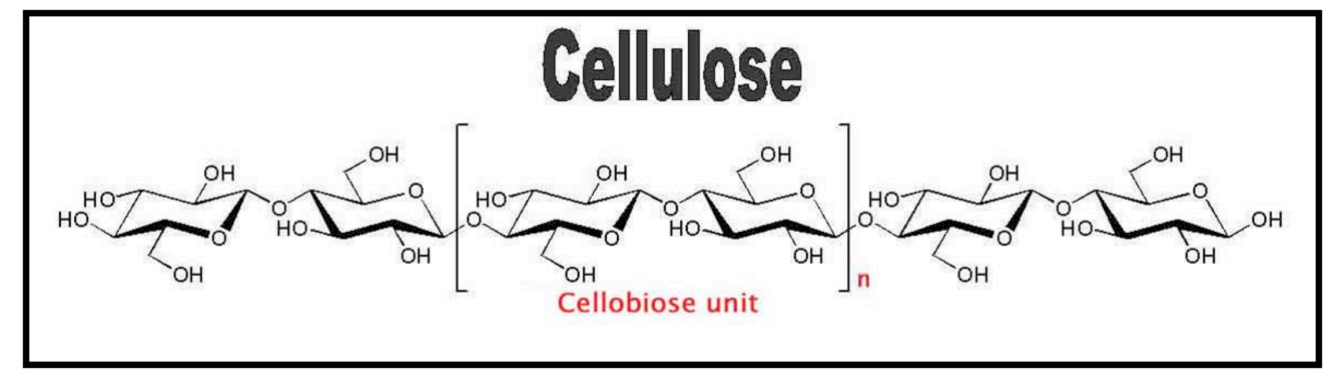

Figure 4: Structure of cellulose (Enchhorn et al., 2010)

Goetz et al. (2009) have attempted to blend polysaccharide nanocrystals with various polymers. The resulting reinforced nanocomposite showed outstanding improvement of mechanical properties. Enchhorn et al. (2010) showed that incorporation of cellulose nano-fibers into a polymeric matrix increased Young's modulus of the nanocomposites up to $138 \mathrm{GPa}$ and the tensile strength to $2 \mathrm{GPa}$ by comparing to the pure polymeric samples with no cellulose. The chain structure of the cellulose gives the opportunity to form hydrogen bonds with the polymeric matrix which results mechanical strength and chemical stability. 
According to Ciobanu et al. (2009), non-polar polymers including PLLA have almost no affinity to the polar reinforcing agents such as HA or cellulose. In other words, cellulose and HA have hydrophilic nature and the PLLA has hydrophobic characteristics, therefore the interfacial bonding between these three as well as the homogenous dispersion in polymeric solution is still a remaining challenge (Wei \& Ma, 2004; Xiao \& Zhang, 2007) The strong affinity of cellulose crystals to each other due to their high surface energy encourage their aggregation and sedimentation (Li et al., 2009; Bondeson et al, 2006). So, treatment of nano fibers and nano particles is a necessary step in nanocomposites fabrications.

\subsection{Treatment of nanosized reinforcing agents}

When it comes to preparation of the nanocomposites, the main challenge is homogeneous dispersion of various reinforcing agents in the matrix and strong interfacial bonding between the constituents and matrix to improve final properties. Two characteristics of nanoparticles make their dispersion difficult.

First of all, their high surface area to volume ratio makes them more chemically reactive compounds, so they tend to agglomerate and reduce their surface energy. Second of all, when the matrix and reinforcing agent have the opposite affinity to water, it is really hard to achieve a homogenous dispersion of a hydrophilic nanoparticles or nanofibres in a hydrophobic matrix. Table 3 shows various approaches that have been tested by numerous researchers to pre-treat the reinforcing agents. 
Various methods have been tested to overcome difficulties to obtain uniform dispersion. A unique combination of the physical, chemical, or mechanical methods developed for fabrication of the nanocomposites of this research.

Table 3: Literature survey related to improve dispersibility of nano-phase agents

\begin{tabular}{|c|c|c|c|c|c|}
\hline Compound & $\begin{array}{c}\text { Approach } \\
\text { Type }\end{array}$ & Treatment & Conditions & $\begin{array}{c}\text { Reaction } \\
\text { Time and } \\
\text { Temp. }\end{array}$ & References \\
\hline MCC & Mechanical & $\begin{array}{c}\text { Ultra } \\
\text { sonication }\end{array}$ & $\begin{array}{c}\text { Dispersion of } \\
\text { MCC in DMF }\end{array}$ & $\begin{array}{c}30 \text { min, Ice- } \\
\text { bath }\end{array}$ & $\begin{array}{c}\text { (Bondeson, } \\
\text { 2006) }\end{array}$ \\
\hline MCC & Chemical & $\begin{array}{c}\text { Acid } \\
\text { Hydrolysis }\end{array}$ & $\begin{array}{c}\text { Sulfuric acid } \\
63.5 \%(\mathrm{w} / \mathrm{w}) \\
\text { to MCC ratio } \\
10 \mathrm{ml} / \mathrm{g} .\end{array}$ & $\begin{array}{c}130 \mathrm{~min}, \\
44^{\circ} \mathrm{C} \\
\text { (hydrolysis) } \\
30 \text { min, ice- } \\
\text { bath Ultra } \\
\text { sonication }\end{array}$ & $\begin{array}{c}\text { (Liu } \text { et al., } \\
2010)\end{array}$ \\
\hline MCC & Physical & $\begin{array}{c}\text { Coupling } \\
\text { agent }\end{array}$ & $\begin{array}{c}\text { Ultra } \\
\text { sonication }\end{array}$ & $\begin{array}{c}15 \text { min, ice- } \\
\text { bath }\end{array}$ & $\begin{array}{c}\text { (Frone, } \\
\text { 2011) }\end{array}$ \\
\hline HA & Mechanical & $\begin{array}{c}\text { Mechanical } \\
\text { shaking } \\
\text { (Vortex) }\end{array}$ & $\begin{array}{c}\text { Ultra } \\
\text { sonication }\end{array}$ & $\begin{array}{c}30 \text { min, ice- } \\
\text { bath. }\end{array}$ & $\begin{array}{c}\text { (Nejati } \text { et } \\
\text { al., 2009) }\end{array}$ \\
\hline
\end{tabular}

\subsection{Water absorption of the nanocomposites}

One of the problems of nanocomposites reinforced with natural fibers that still remains unsolved is the high affinity of natural fibers towards water absorption. Biomaterials containing natural fibers absorb water rapidly which may result in sooner than expected biodegradation (Goetz \& Oksman, 2009). Moisture penetration into the nanocomposite is controlled by three mechanisms. The first mechanism is the diffusion of water molecules 
inside of the micro-gaps between polymer chains. The second common mechanism is capillary transport into the gaps and flaws at the interfaces between fibres and polymer. It is particularly important when the interfacial adhesion is weak, because of incomplete wettability; and the third is transport by micro-cracks in the matrix, formed during the fabrication process (Espert et al. 2004). Espert et al. (2004) investigated the water absorption mechanisms and behaviour of natural fiber/polypropylene composites. They increased the fiber loading of the composites in order to take the advantage of the reinforcing effect of cellulose fibers. But they observed that increasing the content of the fiber in the structure of the composites would enhance their water absorption potential. Because increase of the cellulose content led to higher values of the "effective diffusion coefficients" for all the different blends of their composites. For transport in porous media, "effective diffusion coefficient" is usually used, which varies with the medium, i.e. cellulose content. Due to the hydrophilic character of natural fibers, the entrance of the water molecules inside the nanocomposites affected the kinetics of the diffusion processes. So, another ongoing challenge in terms of natural fiber containing nanocomposites is to control the amount of absorbed water, which affects the rate of polymer degradation. Also, this phenomenon would cause undesired and catastrophic changes in vivo due to swelling and changing the size of the implant and deterioration of the mechanical strength. So, the unfilled gap in literature with respect to natural fiberreinforced composites is improving their water absorption resistance and decreasing their biodegradation rate without sacrificing their mechanical properties especially when the content of natural fiber is increased. 


\subsection{Literature gap}

So far an appropriate alternative for a human bone that has all the advantages of autologous bone without its disadvantages does not exist. Therefore, more research needs to be done to introduce unique combinations of various materials to manufacture novel biomaterials that mimic real bone in terms of chemical, mechanical, thermal, morphological, and biological properties. It is found that the literature lacks sufficient information in the following areas:

- Synthetic bone grafts mimicking certain properties of the real bone have been developed with limited success. The complexity of variables has made their production challenging. So introduction of new biomaterials that have all the advantageous of the autograft bone without the disadvantages of the other alternatives for bone graft substitute is needed.

- Poly L- Lactic Acid (PLLA) based composites and nanocomposites have some drawbacks, including low mechanical properties and acidic degradation byproducts. The $\mathrm{pH}$ at the implantation site (in vivo) will decrease, resulting in necrosis of the implantation site and rejection of the implant.

- One of the ongoing challenges is that polymer-ceramic composite or nanocomposites get more brittle after incorporation of HA and crack under the stress due to the brittle nature of HA. So, in case of load-bearing applications, especially for bone replacements purposes, these composites need to be more ductile and flexible.

- The incompatibility of the reinforcing agents and polymeric matrix in terms of their tendency to water causes non-uniform dispersion of the reinforcing agents in the 
polymeric matrix and weak chemical bonding between them, which will deteriorate the mechanical properties.

- Another challenge is the high tendency of natural fibers such as cellulose fibers to absorb water, which results in sooner than expected biodegradation, an undesired size change of the implant, and unmet mechanical strength in vivo. So, improving the water resistance of these fibers is a necessary task to do.

- Since the mechanical properties of human bone vary tremendously according to the location and function in the body (i.e. load bearing or non-load bearing), the mechanical properties of the artificial bone substitutes or bone grafts should be tailored to match the properties of the surrounding bone at the damaged site. Besides, the biodegradation rate of the device needs to be tuned based on the patient's age, to match the rate of the bone regeneration process. Tailoring the mechanical behaviour and biodegradation rate of the nanocomposite biomaterials is possible by effectively controlling the weight fraction, ratio and the type of the reinforcing agents.

Based on the literature review, combination of MCC with HA nano particles with incorporation of an appropriate coupling agent to improve the overall properties of composite has advantages over other nanocomposites such as:

a) To improve brittleness of the HA containing nanocomposites and increase the elongation at break of the final nanocomposite by employing the cellulose (MCC) in the composition of the nanocomposites. 
b) To enhance the performance of the HA nanoparticles by retarding their migration to surrounding tissue by incorporation of cellulose in vivo (Blitterwijk, 2008).

c) To improve the overall properties of the nanocomposites. To increase the solubility of the HA nanoparticles that have low solubility in implantation site and increase the effectiveness of the implant in vivo cellulose introduced (Blitterwijk, 2008).

d) To improve the water resistance of the cellulose containing nanocomposites by involving and interlocking the hydrophilic groups of the cellulose and HA in chemical bonding.

\subsection{Research objectives}

The main goal of this research is to develop a novel synthetic bone graft biomaterial that has similar biological, mechanical, chemical, and thermal properties than those of real bone. More specifically, the aim is to develop biomimetic nanocomposites from cellulose, which possess properties similar to autologous bone including morphology, composition, microstructure, and mechanical properties. To achieve this main objective, the following sub-objectives under two stages needed to be conducted:

Stage 1: Develop an MA series of nanocomposites by varying the weight ratio of cellulose and hydroxyapatite in Poly L-Lactic Acid to find an optimum range for the weight fraction of each constituent.

1. Improve the dispersion of the cotton source microcrystals (MCC) by disperse them homogenously in a hydrophobic polymer matrix (PLLA) as reinforcing 
agent and modifying the surface to achieve better interfacial bonding with the matrix.

2. Form a homogenous dispersing them of the cellulose microcrystals and hydroxyapatite nanoparticles in PLLA matrix and to improve the interfacial bonding between these reinforcing agents and PLLA matrix.

3. Characterize the chemical, thermal, biological, morphological, and mechanical properties of MA series of the nanocomposites.

4. Improve the mechanical properties of the nanocomposites such as Young's modulus, tensile strength, and elongation at break by changing the weight ratio of reinforcing agents (MCC and $\mathrm{HA}$ ).

5. Find the appropriate range for weight fraction of the constituents of the nanocomposites.

6. Evaluate the toxicity of the novel biomaterial exposure to bone cells.

Stage 2: Optimize the nanocomposites in order to achieve maximum compressive properties and minimum water absorption or mass loss.

7. Design and fabricate an $\mathrm{MH}$ series of nanocomposites by selecting the narrower range of the weight fractions of the constituents from the MA series and by incorporating porogen.

8. Investigate the influence of the cellulose content and hydroxyapatite content solely on mechanical behavior of the MH series of nanocomposites.

9. Create a model that will accurately predict which parameters.

10. Identify the active factors (variables) and measure their effect on each response. 
11. Investigate the effects of the ratio of microcrystalline cellulose to hydroxyapatite nanoparticles, concentration of (PLLA), and porogen content onto the mechanical and water absorption, and biodegradation behaviour of the $\mathrm{MH}$ series of the nanocomposites.

12. Tailor the mechanical properties and biodegradation rate of the nanocomposites used as artificial bone to match the properties of the surrounding bone at the damaged site.

13. Improve the water absorption resistance of the $\mathrm{MH}$ series of nanocomposites and then decrease their biodegradation rate without scarifying their mechanical properties especially when the content of natural fiber is increased.

14. Optimize the process parameters (variables) in order to maximize the compressive properties and minimize the water absorption or the mass loss based on an ANOVA. 


\section{Chapter 3. MATERIAL AND METHODS}

\subsection{Material}

\section{Poly (L-Lactic Acid)}

Poly L-Lactic Acid (PLLA) polymer was purchased from Lactel ${ }^{\circledR}$, USA and directly used as matrix for nanocomposites. The solid yellowish polymer particles had an average molecular weight $\left(\mathrm{M}_{\mathrm{w}}\right)$ of $85000 \mathrm{~g} / \mathrm{mol}$, a glass transition temperature $\left(\mathrm{T}_{\mathrm{g}}\right)$ ranging from 60 to $65{ }^{\circ} \mathrm{C}$, a degradation time beyond 24 months, and $37 \%$ of crystallinity. PLLA was preserved at $-20{ }^{\circ} \mathrm{C}$ (below its $\mathrm{T}_{\mathrm{g}}$ ) to be used upon request.

\section{Cotton sourced microcrystalline cellulose}

Cotton source microcrystalline cellulose (MCC), with a mean particle size of $20 \mu \mathrm{m}$ and an aspect ratio of 2-4, purchased from Sigma-Aldrich (USA) was used as reinforcing agent. MCC was used as a naturally occurring biopolymer to resemble collagen fibers of real bone.

\section{Hydroxyapatite nanoparticles}

The hydroxyapatite (HA) nanoparticles were obtained from Sigma-Aldrich, with a mean particle size $<200 \mathrm{~nm}$ dispersed in water and a surface area $14.3 \mathrm{~m}^{2} / \mathrm{g}$. It was used as bioceramic reinforcing agent to resemble the natural calcium phosphate minerals of real bone.

\section{Sodium Dodecyl Sulphate}

Sodium Dodecyl Sulphate (SDS) is an anionic surfactant that was used as coupling agent and was purchased as white solid particles, from (Fluka, Canada). It used as a coupling 
agent to bind the hydrophilic reinforcing agents (MCC and HA nanoparticles) to the hydrophobic PLLA polymer chains.

\section{Sodium chloride}

Sodium chloride $(\mathrm{NaCl})$ was purchased as solid particles from Fluka, Canada with particle size of 200-300 $\mu \mathrm{m}$ to create pores after leaching out by water.

\section{Organic solvents}

We also used various solvents during the fabrication process of the nanocomposites: 1,4dioxane obtained from Sigma-Aldrich, Canada, with purity $\geq 99 \%$, was used as solvent for PLLA; Ethanol was purchased from VWR, Canada, with purity 99\%; chloroform, hydrochloric acid, and nitric acid were obtained from Sigma-Aldrich, Canada, and deionized distilled water was produced by Chemistry Department of Ryerson University.

\section{Cultured cell lines}

Rat cells line, URM-106 (Osteosarcoma, ATCC) were grown in Dulbecco Minimal Essential Medium (DMEM), supplemented with 10\% fetal bovine serum (FBS), 1\% penicillin streptomycin (PS) and 2 ml-glutamine. This mixture was called cell culture medium. These cells (passages 25-30) were maintained in humidified atmosphere of 5\% $\mathrm{CO}_{2}$ at $37^{\circ} \mathrm{C}$ in cell culturing incubator. The cell culture medium was changed every $48-$ $72 \mathrm{hrs}$. For sub-culturing, the cells were dissociated with $0.25 \%$ trypsin-EDTA (Ethylene di amine tetra acetic acid), which was neutralized with culture medium, and sub-cultured in $75 \mathrm{~cm}^{2}$ flasks. 


\subsection{Methods}

The MA series of nanocomposites was developed as the preliminary set of nanocomposites in order to find the range of the weight fraction of each constituent in the nanocomposites. After developing and characterizing of the MA series of nanocomposites, the weight fraction ranges were used for manufacturing $\mathrm{MH}$ series of nanocomposite using experimental design approach. While the ratio of the MCC over HA was kept constant and equal to 1 in MA series of the nanocomposites, the ratio of the MCC over PLLA and the ratio of the HA to PLLA was varied from 1 to 5 in MA series of the nanocomposites to in order to investigate the effect of cellulose and hydroxyapatite separately onto final properties of the nanocomposites. The concentration of PLLA in its solvent was equal to $10(\mathrm{~g} / \mathrm{cc}) \%$. A unique sequence of the conventional prior art fabrication procedures has been combined to manufacture two series of the nanocomposites. Then in order to investigate the effect of the cellulose weight fraction and HA weight fraction separately onto final properties, the ratio of the MCC over HA varied from 0 to 4 in designation of the $\mathrm{MH}$ series of the nanocomposites. The concentration of the PLLA in fabrication of MH series of the nanocomposites were equal to 10,15 , and $20(\mathrm{~g} / \mathrm{cc}) \%$ to investigate the effect of the concentration of the PLLA onto final properties as well. Method of manufacture of the MA series of the nanocomposites modifies to improve the outcome for fabrication of the MH series of nanocomposites. 


\subsubsection{Fabrication procedure of MA series of nanocomposites}

The preparation of the MA series of nanocomposites involved two main steps: First step is the treatment of reinforcing agents (MCC and HA) and the second step is the fabrication of the nanocomposites. The objective of the treatment of the reinforcing agents was to disperse them homogenously in a polymer solution (PLLA in dioxane) and prevent their agglomeration. The treatment of MCC and HA was done using a combination of two physical and mechanical methods. To eliminate any trace of water from the dispersion of HA nanoparticles, a container containing $25 \mathrm{ml}$ of hydroxyapatite dispersed in water containing $25 \mathrm{~g}$ of hydroxyapatite nanoparticles was placed in a freezer and then the solid bulk was transferred to a freeze dryer and kept there for 3 days. The resulting powder of HA nanoparticles as then used in fabrication process. Then, predefined amounts of MCC, HA, and SDS were transferred into sealed containers and dispersed in 1,4-dioxane. The amount of MCC, HA, and SDS were calculated based on the weight fraction percentage and the desired ratio of MCC to PLLA of each type of nanocomposite specimens.

Table 4 presents the specimen designation information for the MA series of nanocomposites. For instance, based on the information provided in Table 4, for preparation of specimen MA5050, $0.505 \mathrm{~g}$ of PLLA, $0.250 \mathrm{~g}$ of MCC, $0.250 \mathrm{~g}$ of HA, and $0.250 \mathrm{~g}$ of SDS, and 5cc of 1,4-dioxane solvent were used. The ratio of MCC to PLLA as well as HA to PLLA were equal to 0.0 for pristine specimen, equal to 0.1 for MA1010 specimens, equal to 0.3 for MA3030 specimens, equal to 0.4 for MA4040 specimens, equal to 0.5 for MA5050 specimens, and equal to 0.6 for MA6060 specimens. 
Table 4: Specimen nomenclature and composition of MA series of the nanocomposites

\begin{tabular}{|c|c|c|c|c|}
\hline $\begin{array}{c}\text { Specimen } \\
\text { Designation }\end{array}$ & $\begin{array}{c}\text { PLLA } \\
\left(\mathbf{W}_{\mathbf{t}} \%\right)\end{array}$ & $\begin{array}{c}\text { MCC } \\
\left(\mathbf{W}_{\mathbf{t}} \%\right)\end{array}$ & $\begin{array}{c}\text { HA } \\
\left(\mathbf{W}_{\mathbf{t}} \%\right)\end{array}$ & $\begin{array}{c}\text { SDS } \\
\left(\mathbf{W}_{\mathbf{t}} \%\right)\end{array}$ \\
\hline Pristine & 100 & - & - & - \\
MA1010 & 76.9 & 7.7 & 7.7 & 7.7 \\
MA3030 & 52.6 & 15.8 & 15.8 & 15.8 \\
MA4040 & 45.45 & 18.18 & 18.18 & 18.18 \\
\hline MA5050 & 40 & 20 & 20 & 20 \\
MA6060 & 35.5 & 21.5 & 21.5 & 21.5 \\
\hline
\end{tabular}

Then the sealed containers, which contained various concentrations of the MCC were exposed to ultra-sonication in an ice-bath for $30 \mathrm{~min}$ at $4 \mathrm{mV}$ power with a coupling agent (SDS) to decrease the size of the MCC crystals and get homogeneous colloidal dispersion. The particle size of the cellulose microcrystals decreased from $200 \mu \mathrm{m}$ to nano size particles as determined by scanning electron microscopy images (Appendix A). In order to fabricate the nanocomposites, $0.5 \mathrm{~g}$ of PLLA was dissolved in $5 \mathrm{cc}$ of 1,4dioxane with the aid of vortex shaker and water bath at $60^{\circ} \mathrm{C}$. A pristine specimen prepared without incorporation of any reinforcing agent or coupling agent was considered as reference. Then the PLLA solution was mixed in the container containing the dispersed treated MCC/HA/SDS particles. As a result, a stable, homogenous, colloidal suspension of the nanoparticles in PLLA solution was obtained. There was no sign of sedimentation even after leaving the container that contains the colloidal solution for 4 hrs seated at room temperature. The colloidal suspension then was poured in cylindrical containers with a diameter of $15 \mathrm{~mm}$ and height of $30 \mathrm{~mm}$, frozen at $-20{ }^{\circ} \mathrm{C}$ for $2 \mathrm{hrs}$ and transferred to a freeze-dryer at $-54^{\circ} \mathrm{C}$ under vacuum to sublimate the solvent and were 
kept there for $72 \mathrm{hrs}$ to sublimate the 1,4-dioxane. This procedure resulted in a porous nanocomposite. The nanocomposites were placed in flasks containing phosphate buffer saline (PBS) and shaken gently at $100 \mathrm{rpm}$ for 6 hrs to remove any excess SDS from the nanocomposites. Then the nanocomposites were washed with deionized water using a procedure similar to that of SDS. Finally, the nanocomposites were dried in a vacuum oven for 6 hours at $30^{\circ} \mathrm{C}$. The fabricated specimens were stored in a vacuum desiccator for a maximum of 1 week before characterization. In all of the nanocomposites, the concentration of the PLLA in the solvent was kept constant at $0.1 \mathrm{~g} / \mathrm{cc}$.

\subsubsection{Fabrication procedure of MH series of nanocomposites}

The fabrication of MH series of nanocomposites was slightly modified in comparison to the method of fabrication for MA series of the nanocomposites. The first modification was in the step of prevention from agglomeration of MCC and HA nanoparticles. It has been found that it is highly preferable that the HA nanoparticles be as dry as possible. Since the HA nanoparticles are generally hydrophilic in nature, it has been found to be highly preferable to actively dry the HA nanoparticles shortly before dispersing them in 1,4 dioxane. This drying step can be done by any known means, but it has been found that a utilization of the solvent extraction/centrifugation with sequential use of the water, acetone, ethanol and 1,4-dioxane, works better.

The Second modification was adding the coupling agent (SDS) to the mixture of MCC and HA in 1,4-dioxane after ultra-sonication step, not before. The rationale behind this was preventing any possible effect of the ultra-sonication onto intermolecular-bonding between constituents (MCC/HA/SDS). 
The Third modification was the incorporation of salt particles as porogen material to the fabrication process of the nanocomposites. When the salt particles leached out, they left porous nanocomposites structures. This step was added into the fabrication of half of the specimens in order to increase the porosity of the nanocomposites, to control the morphology of the pores and to create inter-connected pores. In addition, the effect of the presence of the porogen on the mechanical properties of the MH series of the nanocomposites was also investigated. The porogen content was $28 \%$ of the total weight of each nanocomposite which is about $40 \%$ of the total weight fraction of the of MCC and HA which was constant and equal to $0.5 \mathrm{~g}$. The nanocomposite specimens were prepared according to the composition designation represented in Table 5.

Table 5: Nomenclature of MH series of nanocomposite specimens without and with the presence of a porogen

\begin{tabular}{|ccccc|}
\hline $\begin{array}{c}\text { Nomenclature of } \\
\text { specimens }\end{array}$ & $\begin{array}{c}\text { PLAA } \\
(\mathbf{W t} \%)\end{array}$ & $\begin{array}{c}\text { MCC } \\
(\mathbf{W t} \%)\end{array}$ & $\begin{array}{c}\text { HA } \\
(\mathbf{W t} \%)\end{array}$ & $\begin{array}{c}\text { SDS } \\
(\mathbf{W t} \%)\end{array}$ \\
\hline MH20 4 N & 57 & 22.5 & 6.5 & 14 \\
MH20 1 N & 57 & 14 & 14 & 14 \\
MH20 0 N & 57 & 0 & 28 & 14 \\
MH20 4 P & 57 & 22.5 & 6.5 & 14 \\
MH20 1 P & 57 & 14 & 14 & 14 \\
MH20 0 P & 57 & 0 & 28 & 14 \\
MH15 4 N & 50 & 26.6 & 6.6 & 16.8 \\
MH15 1 N & 50 & 16.6 & 16.6 & 16.8 \\
MH15 0 N & 50 & 0 & 33.3 & 16.8 \\
MH15 4 P & 50 & 26.6 & 6.6 & 16.8 \\
MH15 1 P & 50 & 16.6 & 16.6 & 16.8 \\
MH15 0 P & 50 & 0 & 33.3 & 16.8 \\
MH10 4 N & 40 & 32 & 8 & 20 \\
MH10 1 N & 40 & 20 & 20 & 20 \\
MH10 0 N & 40 & 0 & 40 & 20 \\
MH10 4 P & 40 & 32 & 8 & 20 \\
MH10 1 P & 40 & 20 & 20 & 20 \\
MH10 0 P & 40 & 0 & 40 & 20 \\
\hline
\end{tabular}




\subsubsection{Design of experiments of MH series of nanocomposites using general factorial design method}

The most important step in design of experiments is the correct selection of the factors (variables) and their levels. The general factorial method was selected to design the experiments for fabrication of the $\mathrm{MH}$ series of the nanocomposites with three factors (variables) including concentration of PLLA in its solvent at 10, 15, and $20(\mathrm{~g} / \mathrm{cc}) \%$, the ratio of MCC over HA nanoparticles of 0,1 , and 4 , and the content of porogen at 0 and $40 \%$ of the weight fraction of MCC and HA nanoparticles in the nanocomposites or $28 \%$ of the total weight of the nanocomposite. The factors and their levels were chosen according to literature review, the preliminary results defined from fabrication and evaluation of the properties of the MA series of the nanocomposites, and some observations during the fabrications of the MA series of the nanocomposites.

For the general factorial design with 3 independent factors, and three replicates for each coded nanocomposite specimen 54 run were employed. The nomenclature of the specimens and their designation based on three factors, and the run sequence of coded

samples is represented in Table 6. Each row of the table represents three runs at a specified condition. In order to minimize the errors, the repetition of each specimen was randomized as determined by the design software.

The design of experiments and the statistical analysis of the results were carried out a trial version of Design-Expert software 7.0.0 (Stat-Ease Inc.). 
Table 6: Nomenclature of the MH series of the nanocomposites

\begin{tabular}{|ccccc|}
\hline $\begin{array}{c}\text { Nomenclature } \\
\text { of specimens }\end{array}$ & $\begin{array}{c}\text { Concentration } \\
\text { of PLLA in } \\
\text { solvent } \\
(\mathrm{g} / \mathrm{cc}) \%\end{array}$ & $\begin{array}{c}\text { Weight } \\
\text { Ratio of } \\
\text { MCC/HA }\end{array}$ & $\begin{array}{c}\text { Presence of } \\
\text { porogen }\end{array}$ & $\begin{array}{c}\text { Coded specimens } \\
\text { based on their run } \\
\text { order }\end{array}$ \\
\hline MH20 4 N & 20 & 4 & $\mathrm{~N}$ & $9,16,38$ \\
MH20 1 N & 20 & 1 & $\mathrm{~N}$ & $1,32,34$ \\
MH20 0 N & 20 & 0 & $\mathrm{~N}$ & $10,13,48$ \\
MH20 4 P & 20 & 4 & $\mathrm{P}$ & $30,36,41$ \\
MH20 1 P & 20 & 1 & $\mathrm{P}$ & $19,44,51$ \\
MH20 0 P & 20 & 0 & $\mathrm{P}$ & $23,40,46$ \\
MH15 4 N & 15 & 4 & $\mathrm{~N}$ & $7,17,52$ \\
MH15 1 N & 15 & 1 & $\mathrm{~N}$ & $5,39,49$ \\
MH15 0 N & 15 & 0 & $\mathrm{~N}$ & $31,35,43$ \\
MH15 4 P & 15 & 4 & $\mathrm{P}$ & $6,14,54$ \\
MH15 1 P & 15 & 1 & $\mathrm{P}$ & $25,33,53$ \\
MH15 0 P & 15 & 0 & $\mathrm{P}$ & $3,28,45$ \\
MH10 4 N & 10 & 4 & $\mathrm{~N}$ & $8,20,47$ \\
MH10 1 N & 10 & 1 & $\mathrm{~N}$ & $15,29,50$ \\
MH10 0 N & 10 & 0 & $\mathrm{~N}$ & $21,27,37$ \\
MH10 4 P & 10 & 4 & $\mathrm{P}$ & $12,18,22$ \\
MH10 1 P & 10 & 1 & $\mathrm{P}$ & $4,24,26$ \\
MH10 0 P & 10 & 0 & $\mathrm{P}$ & $2,11,42$ \\
\hline
\end{tabular}

$\mathrm{N}$ represents that no porogen involved in fabrication process and $(\mathrm{P})$ represent that porogen is involved in fabrication process of the nanocomposites

\subsubsection{Analyses description}

After fabrication of the nanocomposites, their characterization techniques were performed using the following techniques.

\subsubsection{Fourier Transform Infrared Spectroscopy for chemical analysis}

Fourier Transform Infrared Spectroscopy (FTIR) was used to evaluate the chemical interactions between the constituents of the nanocomposites. A spectrometer (Nicolet Nexus 670, Corp, Madison, USA) equipped with a NIC (Ni-In-Cd) detector implemented 
for this purpose. The spectrum was measured between $400-4000 \mathrm{~cm}^{-1}$. The background data for a $\mathrm{KBr}$ blank were subtracted from each spectrum. Nanocomposite samples were mixed with $\mathrm{KBr}$ powder by using pastel and mortar at room temperature. The standard spectral resolution of the instrument was used between $0.16 \mathrm{~cm}^{-1}$ and $0.5 \mathrm{~cm}^{-1}$, which is suitable for most applications.

\subsubsection{Differential Scanning Calorimetry (DSC) for thermal analysis}

To investigate the effect of MCC and HA on thermal behaviours of the PLLA matrix, study was performed on the raw material (PLLA), pristine specimen (sample with no MCC and HA) and MA series of the nanocomposites with different weight fractions of the cellulose and hydroxyapatite. Approximately 6 to $11 \mathrm{mg}$ of each nanocomposite weighed and sealed in a small pan of the DSC instrument and placed in a DSC (Perkin Elmer's, PYRIS 7, USA) working under nitrogen atmosphere. The sample was heated from $30^{\circ} \mathrm{C}$ to $180^{\circ} \mathrm{C}$ at a heating rate of $5^{\circ} \mathrm{C} / \mathrm{min}$ then cooled back down at a cooling rate of $20{ }^{\circ} \mathrm{C} / \mathrm{min}$. Heat flow versus temperature thermographs (heating curve and cooling curves) were obtained from the data that DSC provided for us. Then the crystallinity of the samples was obtained using the heating curve and the cooling curves and by application of the equation (1), (Kong, 2002; Mathew et al., 2006):

$\chi_{c}(\%)=\left[\left(\Delta H_{m}-\Delta H_{c}\right) /\left(\Delta H_{m}^{o} \times w_{i}\right)\right] \times 100$

where $\chi_{\mathrm{c}}$ is the sample crystallinity in percentage, $w_{i}$ is the weight fraction of PLLA in the nanocomposites, $\Delta \mathrm{H}_{\mathrm{m}}$ is the melting enthalpy calculated from area under the peak of heating curve DSC graph, $\Delta \mathrm{H}_{\mathrm{c}}$ is the recrystallization enthalpy in cooling step and $\Delta \mathrm{H}_{\mathrm{m}}^{\circ}$ is the melting enthalpy of pure crystalline PLLA is equal to 93.7 $\mathrm{Jg}^{-1}$ (Kong, 2002; Mathew et al., 2006). To analyze the effect of the reinforcing agents on crystallinity of 
the PLLA, exothermic peak which is responsible for amorphous PLLA regions has been considered between $\left(90-110^{\circ} \mathrm{C}\right)$ at $(1400-1600 \mathrm{sec})$ in order to calculate $\Delta \mathrm{H}_{\mathrm{c}}$ and two endothermic peaks responsible for the melting of crystalline PLLA between (160 - 185 $\left.{ }^{\circ} \mathrm{C}\right)$ at $(2200-2600 \mathrm{sec})$ to calculate $\Delta \mathrm{Hm}^{1}$ and between $\left(50-70{ }^{\circ} \mathrm{C}\right)$ at $(1100-1300 \mathrm{sec})$ to calculate $\Delta \mathrm{Hm}^{2}$. While $\Delta \mathrm{Hm}=\Delta \mathrm{Hm}^{1}+\Delta \mathrm{Hm}^{2}$. DSC analysis were conducted on the MCC, SDS and HA separately to make sure that there is no peak from these raw materials that overlaps with the PLLA peaks. In order to analyze the DSC graphs, normalization of each DSC thermograms is required in order to have the heat flow in Joule per gram of PLLA. The DSC software gives us the heat flow data at joule, in order to normalize it heat flow was divided by the weight of the nanocomposite sample used for DSC and multiplied by weight fraction of the PLLA in selected nanocomposite. The area under a DSC thermograms peak at desired area calculated by the help of the data analysis software of the DSC instrument.

\subsubsection{Scanning Electron Microscopy for morphological analysis}

The microstructure of the nanocomposite samples was examined using a Hitachi 2500 scanning electron microscope (SEM). All samples were coated with a conductive layer of sputtered gold. Micrographs were taken at an accelerating voltage of $15 \mathrm{kV}$ in secondary electron mode to ensure a suitable image resolution. A quantitative analysis of the porosity and pore size was performed by Clemex image analyser software to measure pore size. At least 3 SEM images per sample were taken at 200 and 500 magnifications and used for image analysing of the MA as well as MH series of the nanocomposites. 


\subsubsection{Liquid displacement method of the nanocomposites}

A liquid displacement method was used to measure the porosity of the MH series of nanocomposites samples. The sample nanocomposite were cut to cubic samples with dimension of $(7 \mathrm{~mm} \times 7 \mathrm{~mm} \times 7 \mathrm{~mm})$ then placed in a $12 \mathrm{cc}$ graduated cylinder with $12 \mathrm{cc}$ of ethanol. Ethanol was used as the liquid because it did not induce any reaction or shrinkage, and it penetrated easily into the pores. A measured weight sample (W) was immersed in a cylinder tube containing a known volume of ethanol $\left(\mathrm{V}_{1}\right)$. The cylinder was placed in a vacuum to force the ethanol to penetrate entirely the pores. The total volume of ethanol containing the sample was recorded as $V_{2}$. The ethanol saturated specimens was removed from the tube after $5 \mathrm{~min}$, and then the residual ethanol volume was recorded as $\mathrm{V}_{3}$. The relative porosity of the open pores of the MH nanocomposites was calculated using the equation (2) by Asefnejad et al. (2011).

$\mathrm{P}(\%)=\left(\mathrm{V}_{1}-\mathrm{V}_{2}\right) /\left(\mathrm{V}_{2}-\mathrm{V}_{3}\right)$

\subsubsection{Compression Tests for mechanical analysis}

The compressive strength and Young's modulus of both the MA series and the MH series of the nanocomposites were measured using an Electronic Universal Testing Instrument (United, USA). The tests were performed using a $500 \mathrm{~N}$ load cell and a crosshead speed of $1 \mathrm{~mm} / \mathrm{min}$. The samples were cut from the original nanocomposites by using a sharp cutter to cylindrical specimens with $13 \mathrm{~mm}$ diameter and $20 \mathrm{~mm}$ height. Then the surface of the specimens was prepared using rotating plates in order to complete contact of the specimen surface with compression jigs. The compressive yield strength was defined as the cross point of the two tangents in the stress-strain curve around the yield point. The 
Young's modulus was calculated from the slope of the initial linear stress versus strain plot at strain $<0.002$. Three samples were tested for each type of the nanocomposite.

\subsubsection{Water Absorption test}

The samples cut from original MH series of the nanocomposites to cubic shape with dimension of $(7 \mathrm{~mm} \times 7 \mathrm{~mm} \times 7 \mathrm{~mm})$; then dried and weighed before placing them in sealed cylindrical containers with $15 \mathrm{~mm}$ in diameter and $40 \mathrm{~mm}$ height, and filled with distilled water where the ratio of mass of sample to water volume was 1:100 (w/v). They kept in water bath for 330 hours at room temperature in an incubator. At each time point, $(0,36,72,120,264$, and $330 \mathrm{hrs})$ samples were taken out from the water, rinsed with distilled water, gently wiped out with Climex napkins, and then weighed with a sensitive microbalance. The water absorption was calculated according to equation (3):

Water absorption $(\%)=\left(m_{2}-m_{1}\right) / m_{1} \times 100$

where, $\mathrm{m}_{1}$ is the initial weight of dry sample, and $\mathrm{m}_{2}$ is the weight of wet sample at any time (Asefnejad et al. 2011).

\subsubsection{Mass Loss analysis for calculation of the degradation rate}

The samples taken from MH series of the nanocomposites that were prepared for the water absorption test, used for the mass loss experiment as well. The nanocomposite to water ratio was kept at 1:100 $(\mathrm{w} / \mathrm{v})$ at room temperature. The culture medium was changed every week. Samples remained in water for 3 months and were weighted after $1,3,5,7,9,17,53$, and 87 days. Samples were rinsed with deionized water, completely dried in a vacuum oven at $30^{\circ} \mathrm{C}$, and weighed. The mass loss was calculated by comparing the original sample mass with that at given amount of time, as shown in equation (4): 
Mass loss $(\%)=\left[\left(M_{0}-M_{t}\right) / M_{0}\right] \times 100$

where $\mathrm{M}_{0}$ is the initial weight of the nanocomposite at each time point, and $\mathrm{M}_{t}$ is the final weight of nanocomposites after extracting from water.

\subsubsection{MTT assay to evaluate the cytotoxicity of the nanocomposites}

MTT is a calorimetric assay for assessing cell metabolic activity. NAD(P)H-dependent cellular oxidoreductase enzymes reflect the number of the viable cells present, under defined conditions. These enzymes are able to reduce the tetrazolium dye MTT 3(4,5dimethylthiazol-2-yl)-2,5-diphenyltetrazolium bromide, which has a purple color. Tetrazolium dye assays herein used to measure cytotoxicity (loss of viable cells) in presence of the potential toxic materials (ISO 10993-5, 2009).

Nanocomposite samples (eight) were cut to cylindrical shapes with diameter of $13 \mathrm{~mm}$ and length of $2 \mathrm{~mm}$. They were sterilized by autoclaving them at $121^{\circ} \mathrm{C}$ for 45 minutes at 2 atm. Each sterilized and cooled nanocomposite sample was immersed in DMEM. DMEM is a modification of Basal Medium Eagle (BME) that contains a four-fold higher concentration of amino acids and vitamins, as well as additional supplementary components. The original DMEM formula contains $1000 \mathrm{mg} / \mathrm{L}$ of glucose and was first reported for culturing embryonic mouse cells as an extracting media with an extraction medium volume to surface area ratio of $1.25 \mathrm{ml} / \mathrm{cm}^{2}$ at $5 \% \mathrm{CO}_{2}$ and $37{ }^{\circ} \mathrm{C}$. After $24 \mathrm{hrs}$, the rich liquid was removed and centrifuged before using. Rat cells were detached from the culture using Trypsin/EDTA (Ethylenediaminetetra acetic acid) and subsequently pelleted by centrifugation for $5 \mathrm{~min}$ at $2000 \mathrm{RPM}$. Cells were re-suspended to a concentration of $1 \times 10^{4}$ cells $/ \mathrm{ml}$, and $100 \mu$ of this suspension was added per well to a 96 well plate. Cells were cultured for $24 \mathrm{hrs}$ at $37{ }^{\circ} \mathrm{C}$ in a $5 \% \mathrm{CO}_{2}$ atmosphere before the 
medium of each well was aspirated. Extraction media were added to the wells and maintained at $37{ }^{\circ} \mathrm{C}$ in a $5 \% \mathrm{CO}_{2}$ atmosphere. Control samples consisted of URM-106 cells grown on tissue culture plastic (TCP) supplemented with DMEM. After 24 hrs of incubation, the supernatant liquid was removed from each well, and the adhering cells were treated with an MTT solution for $4 \mathrm{hrs}$ at $5 \% \mathrm{CO}_{2}$ and $37^{\circ} \mathrm{C}$. The supernatant liquid was removed, $100 \mu \mathrm{L}$ of Dimethyl Sulfoxide (DMSO) was added to each well, and the plate was shaken with flat-surfaced vortex shaker for $5 \mathrm{~min}$ before reading at $590 \mathrm{~nm}$ on a micro plate reader (PowerWaveX; Bio-Tek Instrument). Toxicity was calculated based on the percentage of control-cell viability (ISO 10993-5, 2009). By this measurement, the toxicity and cell viability in the presence of the biomaterial extract can be evaluated. The same steps were repeated after 48 and 72 hrs incubation time. 


\section{Chapter 4. RESULTS AND DISCUSSION}

\subsection{Physiochemical, thermal, and mechanical properties of the MA series of the nanocomposites}

It was not possible to produce nanocomposites without any treatment step or without incorporation of a coupling agent, because MCC and HA nanoparticles were mixed, they showed tendency to sedimentation at the bottom of the test tube. This sedimentation resulted specimens with really weak properties. They collapsed and were even destroyed with simple finger pressure. In order to tackle this problem, treatment step including ultra-sonication and adding SDS as a coupling agent added to the fabrication process. The result was a stable colloidal suspension of MCC, SDS, and HA nanoparticles in the PLLA solution. Figure 5-b shows this homogenous colloidal mixture achieved at the end of the treatment step.

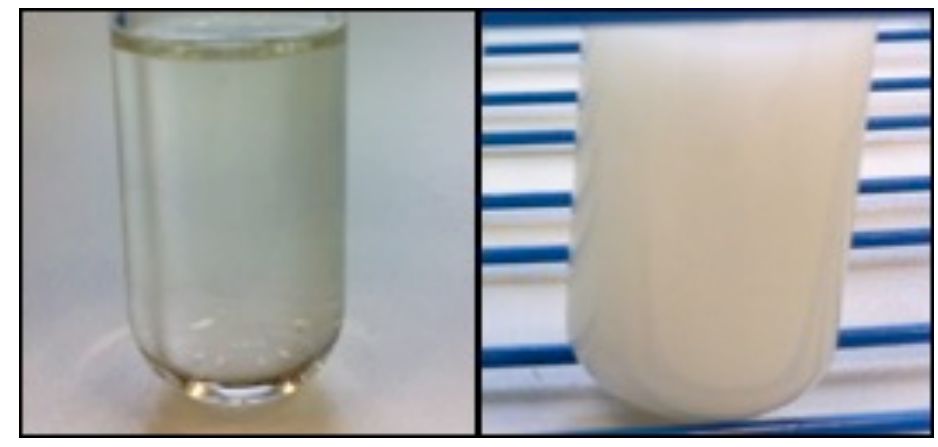

Figure 5: (a) Transparent PLLA solution in 1,4-Dioxane. (b) Homogenous colloidal dispersion of MCC in PLLA solution in the presence of SDS as coupling agent

The chemical reaction behind this phenomenon is that the hydrophilic head of SDS absorbed to the cellulose surface, whereas its hydrophobic tail attached to the PLLA. Besides, the SDS is able to bond to $\mathrm{Ca}^{2+}$ through ionic phosphate and carboxylate groups 
of the hydroxyapatite. So, the final homogenous dispersion of the nanoparticles may be due to intermolecular interactions and chemical bonding between SDS and MCC, HA, and PLLA. These chemical interactions validated by FTIR characterization.

\subsubsection{Chemical analysis results of MA series of nanocomposites}

In order to evaluate the chemical interactions between the PLLA, the MCC and SDS, a FTIR characterization instrument was utilized. Figure 6 shows the FTIR spectra of the pristine specimen and the MA1010 nanocomposites. The intensity (the area under the peak) of the hydroxyl functional groups $\left(-\mathrm{OH}^{-}\right)$, which is absorption peak of MCC at $3433 \mathrm{~cm}^{-1}$, decreased after presence of SDS. These changes may be attributed to the consumption of the hydroxyl groups by formation of hydrogen bonding between $-\mathrm{OH}$ groups of PLLA, MCC, and HA with functional groups $\left(\mathrm{Na}^{+}\right)$of the SDS. On the other hand, the peak height at $3433 \mathrm{~cm}^{-1}$ is proportional to the concentration of free $-\mathrm{OH}$ groups on MCC that are being reduced by the formation of ionic bonds with $\mathrm{Na}^{+}$of SDS. The wave numbers between $900-1200 \mathrm{~cm}^{-1}$ are attributed to the phosphate functional groups. The peaks at 866,1411 , and $1457 \mathrm{~cm}^{-1}$ are ascribed to carbonate groups and a broad peak at $3571 \mathrm{~cm}^{-1}$ belongs to hydroxyl (-OH) groups in HA (Pavia et al. 2009). It can be noticed from comparing the dashed lined graph with the graph below it, the $\mathrm{PO}^{3-}{ }_{4}$ peaks of HA at 563, 602, 958, 1033 and $1092 \mathrm{~cm}^{-1}$ shifted to the right to $561,601,954$, $1031,1084 \mathrm{~cm}^{-1}$ in the MA series of the nanocomposites.

Peak shifting in FTIR spectra indicate some molecular interactions between HA and PLLA. It can be concluded that the coupling agent encouraged PLLA and HA and MCC to ionize during the nanocomposite formation procedure. For instance, the $\mathrm{COO}^{-}$of the 
PLLA would bind with $\mathrm{Ca}^{2+}$ of HA by the aid of the SDS and new carboxyl-calciumcarboxyl $\left(\mathrm{COO}^{-}-\left[\mathrm{Ca}^{2+}\right]-\mathrm{COO}^{-}\right)$linkage may be formed. These chemical linkages can greatly affect interfacial bonding and amount of hydrogen bonding between (-OH) and ($\mathrm{COOH}$ ) functional groups. All these molecular interactions are believed to increase the chemical compatibility of the MCC and HA nanoparticles with the PLLA matrix, which predicted to increase the crystallinity and subsequently the improvement of the mechanical properties of the MA series of the nanocomposites.

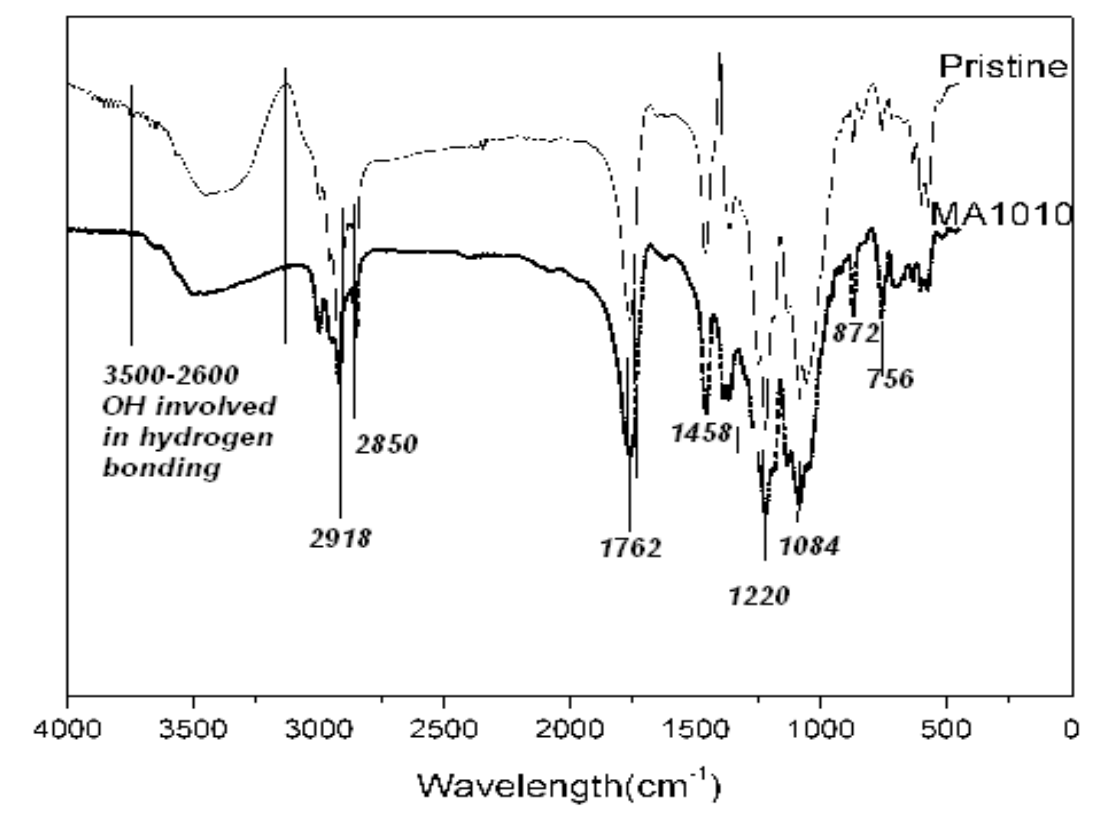

Figure 6: FTIR spectra of pristine specimen and of MA1010 nanocomposite

\subsubsection{Crystallinity of the MA series of nanocomposites}

Figure 7 demonstrates the DSC thermograms, which shows the changes in the thermal behaviour of the MA series of the nanocomposites with various compositions. 


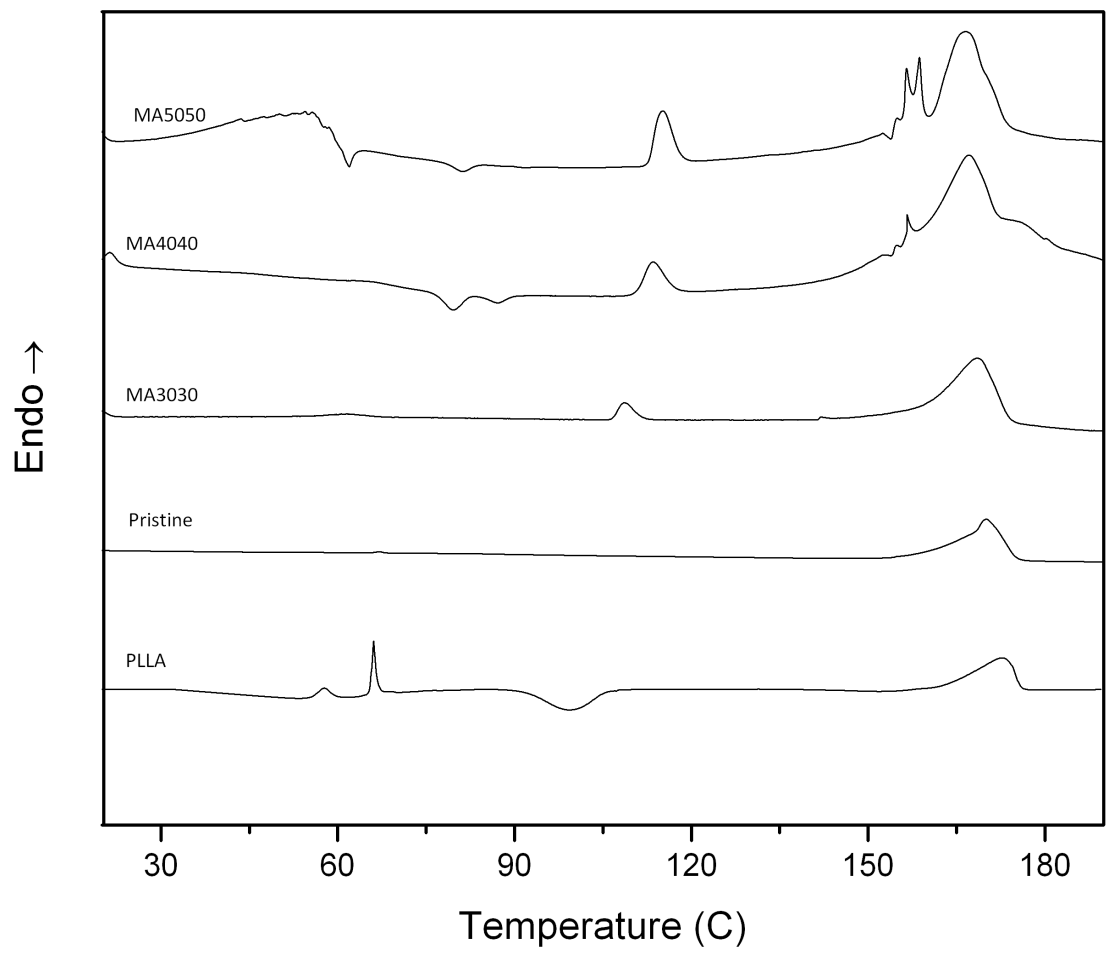

Figure 7: DSC heating scans of the PLLA, pristine specimen, and the MA series of nanocomposites

It is further interesting to note that by increasing the weight fraction of the MCC and HA in the nanocomposites, from bottom to top in Figure 7, the single melting peak typical of the $\alpha$-crystalline phase appearing between $\left(150-180^{\circ} \mathrm{C}\right)$, changes to a double melting peak. This indicates the melting of both the $\alpha^{\prime}$ - and $\alpha$ crystalline phases. The intensity of the exothermic peak at cooling curve $\left(90-110^{\circ} \mathrm{C}\right)$, clearly decreased and finally disappeared with increasing the content of the MCC and HA. Since this region of the peak is related to the melting of the amorphous regions of the PLLA, it can be another evidence to confirm the increase of the crystallinity of the MA series of the 
nanocomposites by increasing the content of the MCC and HA in them. The crystallinity of PLLA increased up to $38 \%$ by increasing the content of MCC and HA. The original DSC thermograms that were used for calculation of the crystallinity were shown in Appendix A. The calculated $\chi_{\mathrm{c}}$ from the thermograms are reported in Table 7.

Table 7: Crystallinity of the raw PLLA, pristine sample, and MA series of nanocomposites

\begin{tabular}{|l|l|l|l|l|}
\hline Specimen & $\mathrm{W}_{\text {PLLA }}(\%)^{*}$ & $\Delta \mathrm{Hm}(\mathrm{J} / \mathrm{g})$ & $\Delta \mathrm{Hc}(\mathrm{J} / \mathrm{g})$ & $\chi(\%)$ \\
\hline Raw PLLA & 100 & +47 & -12.5 & 37 \\
\hline Pristine & 100 & +54 & -45 & 9 \\
\hline MA3030 & 53 & +29 & -23 & 12 \\
\hline MA4040 & 45.45 & +27 & -18 & 21 \\
\hline MA5050 & 40 & +30 & -15.7 & 38 \\
\hline
\end{tabular}

*Data corrected for the percentages of the PLLA in nanocomposites

The results show the effect of increasing the weight ratio of MCC and HA on the crystallinity of the nanocomposites, which may have caused by higher amount of ordered bonds via hydrogen bonding leading to an increase of the crystallinity. The area under the endothermic peak at $\left(150-180^{\circ} \mathrm{C}\right)$ clearly increased with increasing the content of MCC and HA from bottom to top thermograms. This region is related to the melting of the crystalline regions of the PLLA. So, it can be concluded the MCC and HA increased the crystalline regions of the PLLA in the nanocomposites. The crystallinity of the raw PLLA is about $37 \%$, which decreased to $9 \%$ in pristine specimen (Table 7). It may attributable to the negative effect of solving the polymer in its solvent and irregular orientation of crystallites during freezing. However, by incorporation of the HA and MCC in the structure of the nanocomposite, this effect was compensated. This can be related to the presence of the HA nanoparticles that acted as nucleation agents for re-crystallisation of 
the PLLA (Kong, 2002; Mathew et al., 2006). Table 7 lists the calculated $\chi_{c}$ from the analyzed thermograms. The increase in the $\Delta \mathrm{H}_{\mathrm{m}}$ values shows the effect of increasing the weight ratio of MCC and $\mathrm{HA}$ on the crystallinity of the nanocomposites, which may cause higher amount of ordered bonds via hydrogen bonding. Figure 8 shows the typical DSC thermogram of MA5050 used to calculate crystallinity of the nanocomposites. The area under the peak between $170-180^{\circ} \mathrm{C}$ in the heating curve indicates the melting of crystalline phase of PLLA (Figure 8).

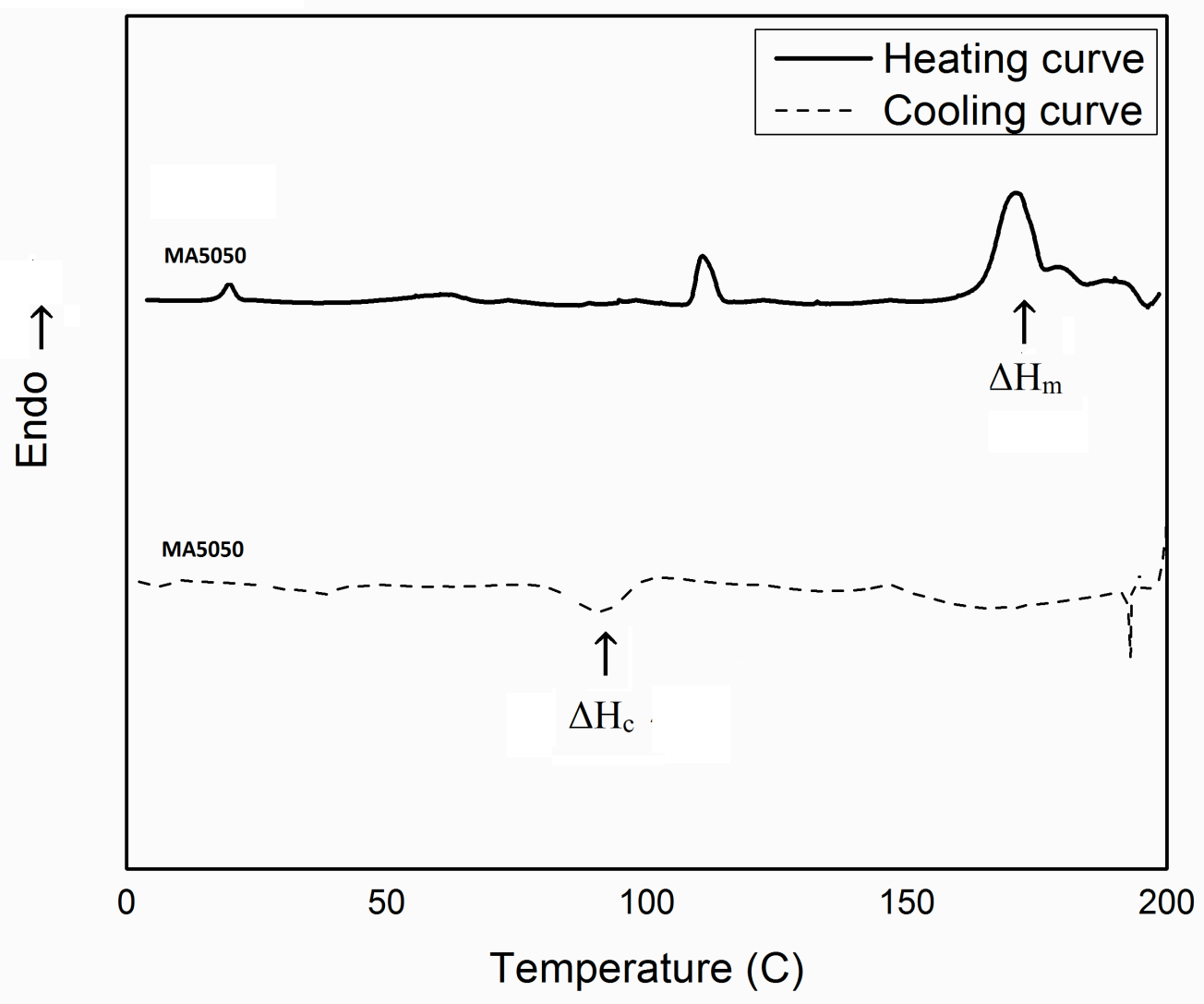

Figure 8: DSC heating and cooling curves of the MA5050 nanocomposite 


\subsubsection{Compressive strength of the MA series of nanocomposites}

Typical stress versus strain curves for the MA series of the nanocomposites are presented in Figure 9. The compressive strength and Young's modulus of the MA series of nanocomposites showed a rising trend with higher weight fraction of the MCC and HA, and PLLA.

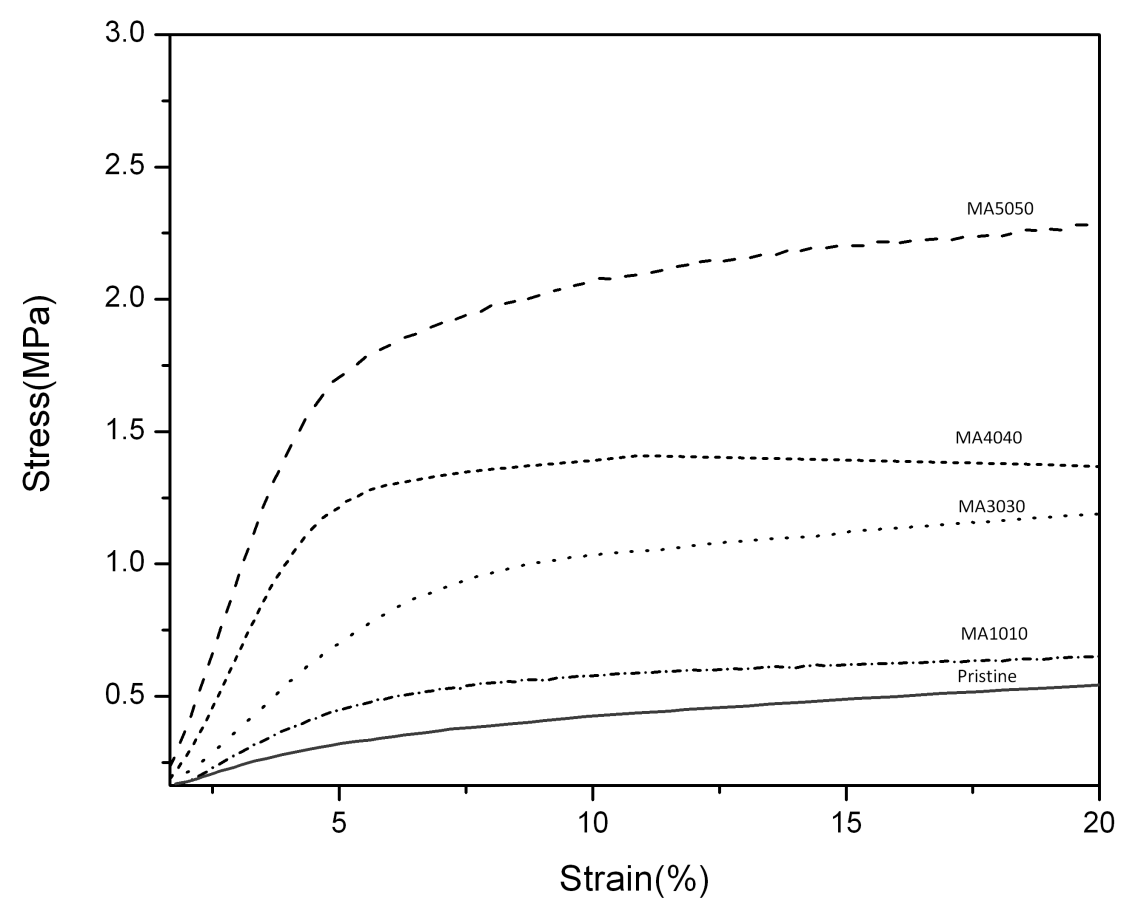

Figure 9: Typical stress-strain curve of MA series of nanocomposites

The values of the compressive strength and Young's modulus of the MA series of the nanocomposites extracted from the typical stress-strain curves represented in Figure 9. The weight ratio of MCC over PLLA increased from 0.1 to 0.5 for MA1010 to MA5050 from bottom to top as depicted in Figure 9. This led to an improvement in the 
compressive yield stress from 0.127 (Pristine PLLA) to 2.2 MPa (MA5050) and the Young's modulus from 6.6 (Pristine PLLA) to $38 \mathrm{MPa}$ (MA5050), respectively. Increasing HA content of the composites may increase crystallinity of the nanocomposites due to the presence of ordered hydrogen bonding and the ceramic nature of the HA leading to higher mechanical properties. The values of the compressive strength and modulus of the composites are reported in Figure (10.a) and Figure (10.b). These values extracted from typical stress-strain curves like the ones were chosen for Figure 9.

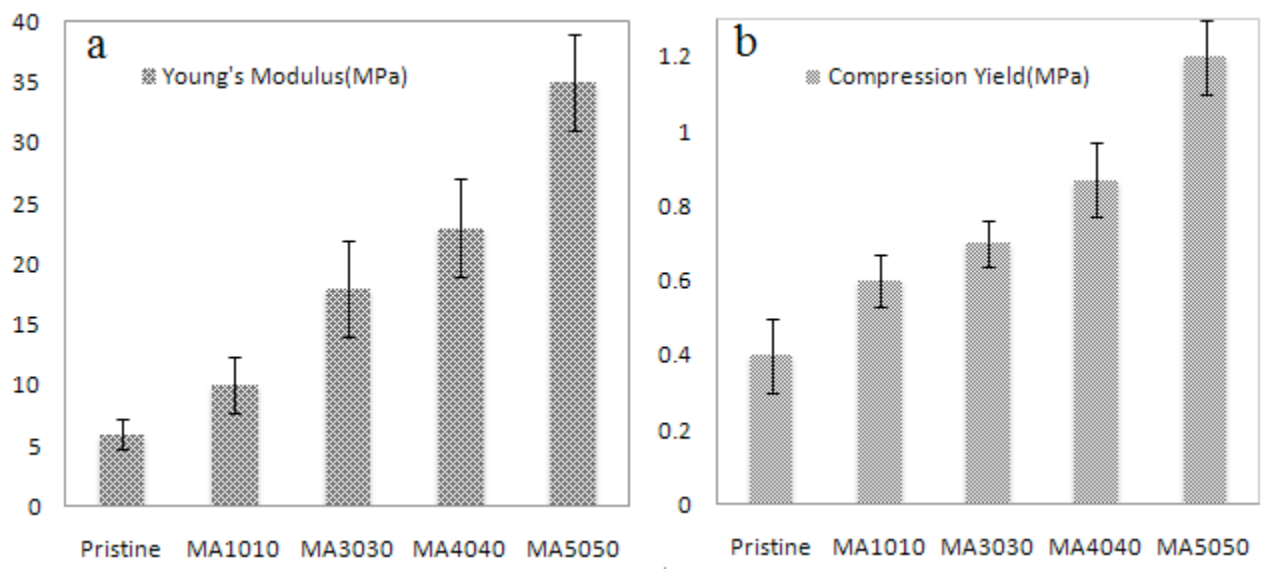

Figure 10: (a) Young's modulus (b) Compression yield of the MA series of nanocomposites

The compressive strength and modulus of the composites represented a rising trend with higher ratio of MCC and HA to PLLA. Increasing of the weight ratio of MCC and HA to PLLA from 0.1 to 0.5 , led to an improvement in the compressive yield stress from 0.127 for pristine PLLA to 1.2 MPa for MA5050 and the Young's modulus from 6.6 (Pristine PLLA) to $38 \mathrm{MPa}$ (MA5050), respectively. Thus the increase of the weight ratio of MCC 
and HA to PLLA from 0 to 0.5 enhanced the compression yield stress by $90 \%$ and the Young's modulus of the nanocomposites by $82 \%$. This significant increase may be attributed to hydrogen bonding between the reinforcing agents (MCC and HA) and the PLLA matrix, as mentioned earlier.

\subsubsection{Morphological observations}

The morphology and microstructure of the MA series of the nanocomposites were examined using SEM as shown in Figure11. The pristine sample (Figure 11.a) seems to be an internal porous structure formed by thermally induced phase separation. The SEM micrographs presented in Figure (11.b) and Figure (11.c) are for MA3030, MA5050 respectively, revealed that HA nanoparticles and $\mathrm{MCC}$ fibers are distributed in the matrix randomly and homogeneously, i.e. some are embedded in pore walls and some piled between pores and there was no sign of agglomeration. 


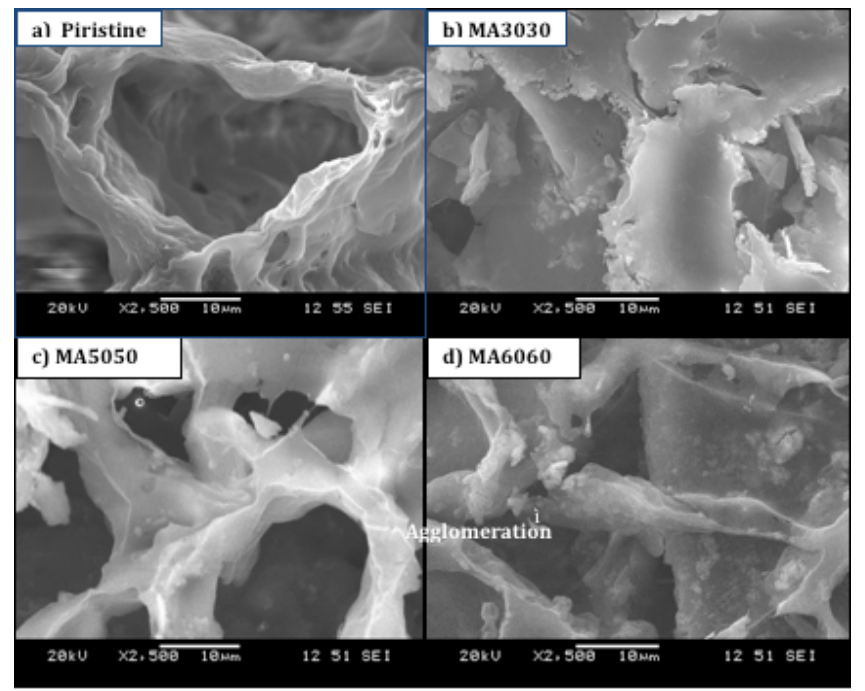

Figure 11: SEM micrographs of (a) Pristine, (b) MA3030, (c) MA5050, (d) MA6060 nanocomposites

Furthermore, by increasing the ratio of HA and MCC to PLLA in composition of nanocomposites, the average pore size value in nanocomposites is reduced in comparison with the pristine sample or with nanocomposites with lower amount of HA and MCC ratios. Some agglomeration in the MA6060 nanocomposite was found (Figure 11-d). This agglomeration affected the mechanical properties of theses nanocomposites and makes them brittle. The agglomeration may be attributed to the consumption of the functional groups of PLLA, so an optimum weight fraction of the MCC and HA to PLLA is needed to obtain nanocomposites with improved mechanical properties.

In order to measure the pore size and porosity of the MA series of the nanocomposites, the SEM micrographs at 200 times magnification were analyzed after adjusting the contrast and applying a threshold of the level of dark as shown in the Figure 12-b which is for the MA4040 (Figure 12-a) nanocomposite after examination by Clemex Image analysis software. The pore size of the MA nanocomposites was in the range of 90- 
$2500 \mu \mathrm{m}$. The image analysis results of the pristine, MA4040, and MA5050 samples are presented at Appendix B.
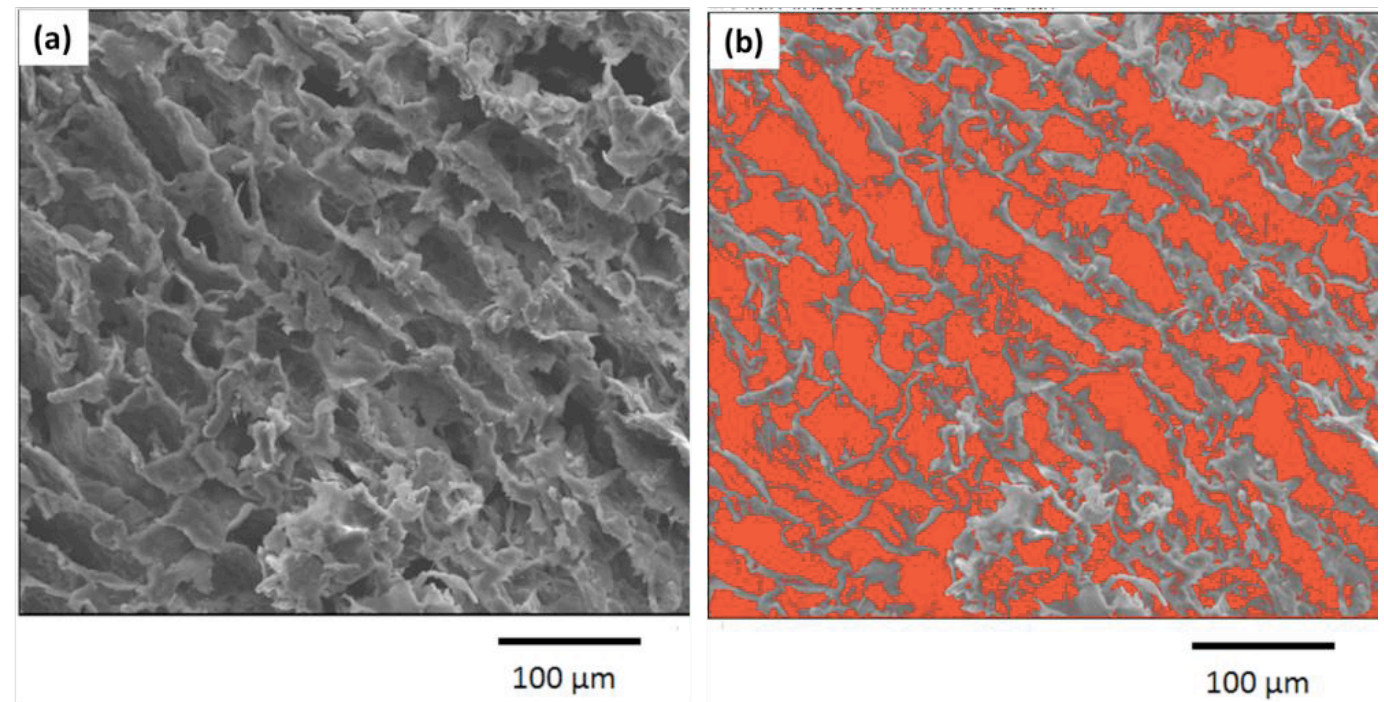

Figure 12: SEM micrographs illustrating a representative cross-section of MA4040 nanocomposite (a) raw micrograph (b) the estimated porous areas

Statistical data showed that the MA series of the nanocomposite had 50-60\% porosity. The required porosity for simulating real bone structure is higher than $75 \%$. Also, the pore size and pore morphology of the MA series of nanocomposites needed more improvement as well. So, in designation of the $\mathrm{MH}$ series of the nanocomposites, porogen introduced to the fabrication process. The image of the micro-plates used for MTT toxicity test have been shown in Appendix C.

\subsubsection{Toxicological studies of the MA series of nanocomposites}

Viability of the cells represented in Figure 13 after 24, 48, and $72 \mathrm{hrs}$ exposure of cells to three sample test groups (extracts) and control sample (with no extract). Cell viability generally was not affected even after 24, 48 and $72 \mathrm{hrs}$ incubation time for all extracts 
compared to the control. For MA4040, and MA5050 nanocomposites, proliferation of the cells caused increasing the population of the cells.

Increasing the number of cells up to $700 \%$ of the control sample, demonstrate the tested nanocomposites high potential to encourage cells to proliferate and growth. Toxicity results shown at Figure 13 proved the absence of toxicological effect of novel nanocomposites on cultured cells even after 48 and $72 \mathrm{~h}$ incubation times. This may be attributed to the osteoconductivity effect of HA and bioactivity of cellulose.

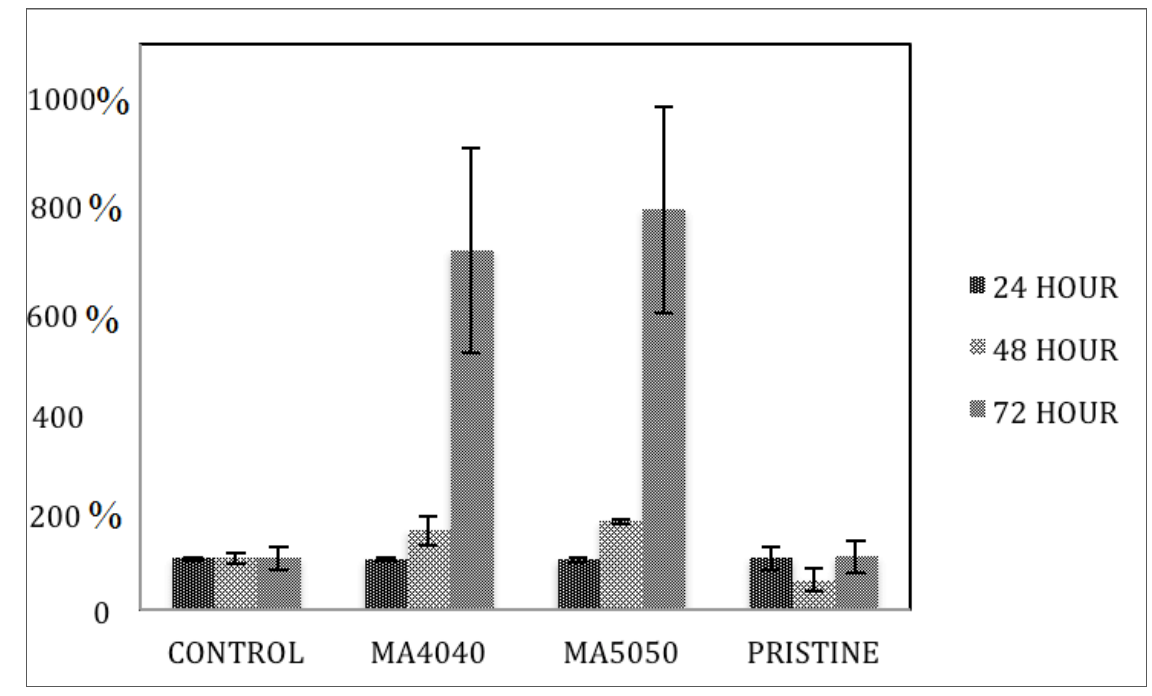

Figure 13: Cells viability of rat cells after incubation in extracts of MA4040, MA5050, and pristine nanocomposites for 24,48 , and $72 \mathrm{~h}$. Control group without extract

\subsection{Optimization of the properties of the MH series of nanocomposites}

In the first stage of this study, the MA series of the nanocomposites were introduced, which were novel series of nanocomposites composed of PLLA reinforced with cotton sourced microcrystalline cellulose (MCC) and hydroxyapatite nanoparticles (HA). Then 
the properties of the MA series of the nanocomposites evaluated to move the research to the second stage. In the second stage of this study, the MH series of the nanocomposites were designed and developed by using the preliminary results obtained from characterization of the MA series of the nanocomposites. In other words, the main goal of the designation of the $\mathrm{MH}$ series of the nanocomposites was to improve and then optimize the properties of them such as mechanical properties, water absorption amount or biodegradation rate of the nanocomposite by identifying the optimum conditions for the process variables.

In this stage of the study the attempts had been made to alter systematically the composition and porosity of the $\mathrm{MH}$ series of the nanocomposites to assess the contribution of the components weight ratio and weight fraction onto mechanical properties, water absorption and mass loss of the nanocomposites. In other words, identifying the contribution of each factor on evaluated property (response) was the first objective of this stage. The second objective of this stage of research was to improve the water absorption resistance of the $\mathrm{MH}$ series of the nanocomposites and then tailoring their biodegradation rate without sacrificing their mechanical properties especially when the content of natural fiber was increased. The third objective of this stage of research was to optimize the process parameters (variables) in order to maximize the compressive properties and minimize the water absorption or the mass loss of the MH series of nanocomposites after analysis of variances by utilization of ANOVA and obtaining predictive equations. 


\subsubsection{Analysis of variances (ANOVA)}

The analysis of variances (ANOVA) is a powerful technique in the general factorial design method that explores the contribution of the affecting factors onto evaluated responses properties. The strategy of ANOVA statistical analysis is to extract the contribution of each factor (variable) onto each evaluated response. The statistical analysis of the results carried out with Stat-Ease Design Expert v.7 software (DX7) trial (Stat-Ease. Inc.).

\subsubsection{ANOVA of compressive yield}

Tables 8,10, and 12 show the ANOVA statistical terms for compressive yield, and Young's modulus, and mass loss of the MH series of the nanocomposites, respectively. In ANOVA tables, $p$-value lower than 0.05 signifies with $95 \%$ confidence that the variation did not occur by chance. Therefore, a factor with the $p$-value higher than 0.05 are assumed to have insignificant effect on the evaluated response and can be deleted from the model. In these tables, "mean square" estimates of the term variance, calculated by the term "sum of squares" divided by "degree of freedom". "Pure error" is the amount of variation in the response in replicated design points (Joulazadeh \& Navarchian, 2009).

Based on the ANOVA of the compressive yield response results, factors $(\mathrm{A}, \mathrm{B}, \mathrm{C}$, and AC) represented the $p$-value less than 0.0001 . So, they affected the compressive yield response significantly. On the other hand, factors $(\mathrm{AB}, \mathrm{BC}$, and $\mathrm{ABC})$ showed the $p$ value higher than 0.0500 at the ANOVA table, which indicated that they did not affect the compressive yield significantly. So the factors $\mathrm{AB}, \mathrm{BC}$, and $\mathrm{ABC}$ reduced from the model safely and the model corrected. 
After correction of the model with reduction of the insignificant factors, the final ANOVA table represented in Table 8. The model F-value of 102.64 implies the model is significant and there is a $0.01 \%$ chance that this large F-value could occur due to noise. Also, the "lack of fit F-value" of 1.00 related to "residual" implies that lack of fit is insignificant, which means that the model fit the data well.

Table 8: ANOVA table after model correction of compressive yield $\left(\sigma_{\mathrm{y}}\right)$ of the $\mathrm{MH}$ series of the nanocomposites

\begin{tabular}{|lccccc|}
\hline Factors & DOF $^{\mathrm{a}}$ & $\begin{array}{c}\text { Sum of } \\
\text { square }\end{array}$ & $\begin{array}{c}\text { Mean square } \\
\text { (Variance) }\end{array}$ & F-value & $\rho$-value \\
\hline Model & 7 & 5.79 & 0.83 & 102.64 & $<0.0001$ \\
Concentration of PLLA & 2 & 2.19 & 1.09 & 135.68 & $<0.0001$ \\
(A) & & & & & \\
Ratio of MCC/HA (B) & 2 & 2.83 & 1.42 & 175.66 & $<0.0001$ \\
Porogen content (C) & 1 & 0.43 & 0.43 & 53.15 & $<0.0001$ \\
AC & 2 & 0.34 & 0.17 & 21.34 & $<0.0001$ \\
& & & & & \\
Residual & 36 & 0.29 & $8.061 \mathrm{E}-003$ & - & - \\
Lack of fit & 36 & 0.29 & $8.061 \mathrm{E}-003$ & & \\
Pure error & 79 & 6.41 & $8.061 \mathrm{E}-003$ & 1.00 & 0.5000 \\
\hline
\end{tabular}

${ }^{\mathrm{a}}$ Degree of freedom

The F-value of each response has been calculated by ANOVA statistical analysis. For instance, by comparing the F-values presented in Table 9, the most influential factor (variable) onto compressive yield response is the ratio of $\mathrm{MCC} / \mathrm{HA}$ (factor $\mathrm{B}$ ) because it showed the highest F-value equal to 175.66, and then the concentration of PLLA (A) with a F-value equal to 135.68. The porogen content (C) with an F-value of 53.15 on the compressive yield. Table 9 represents the summary of the statistics for the compressive yield response of the nanocomposite. 
Table 9: The summary of the statistics for compressive yield of the of the MH series of nanocomposites

\begin{tabular}{|c|c|c|c|c|c|c|}
\hline Response & STD & Mean & $\mathrm{R}^{2}$ & Adjusted $\mathrm{R}^{2}$ & Predicted $\mathrm{R}^{2}$ & $\begin{array}{c}\text { Adequate } \\
\text { Precision }\end{array}$ \\
\hline $\begin{array}{c}\text { Compressive } \\
\text { Yield }\end{array}$ & 0.090 & 0.71 & 0.9089 & 0.9001 & 0.8665 & 37.490 \\
\hline
\end{tabular}

The " $\mathrm{R}$ " is the measure of the amount of variation around the mean explained model.

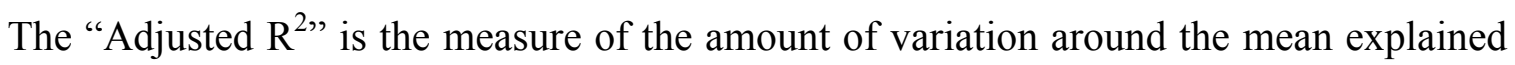
by the model. The "Predicted $\mathrm{R}^{2 "}$ is a measure of the amount of variation in new data

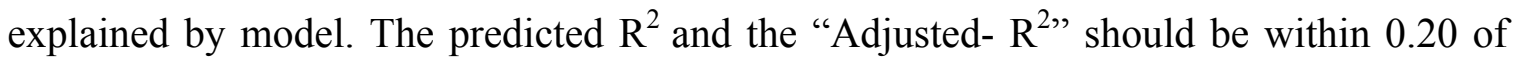
each other. Based $\mathrm{n}$ the data represented in Table 9 can be seen that the "predicted Rsquared" of 0.8665 is in agreement with the "Adjusted $\mathrm{R}^{2 "}$, of 0.9001 . "Adequate precision" measures the signal to noise ratio. A ratio greater than 4 is desirable. Adequate precision ratio of 37.490 indicates an adequate signal, which means the model, can be used to navigate the design space.

\subsubsection{ANOVA of Young's modulus response}

Based on the ANOVA of the Young's modulus response, factors (A, B, C, AC and ABC) represented the $p$-value less than 0.0001 . So, they affected the Young's modulus response significantly. On the other hand, factors (AC and $\mathrm{BC}$ ) showed the $p$-value higher than 0.0500 at the ANOVA table, which indicated that they did not affect the Young's modulus significantly. So the factors $\mathrm{AC}, \mathrm{BC}$ deleted from the model safely and the model corrected. After correction of the model with reduction of the insignificant factors, the final ANOVA table represented in Table 10. The "Model F-value" of 73.5 implies the 
model is significant and there is a $0.01 \%$ chance that this large F-value could occur due to noise. Also, the "lack of fit F-value" of 1.00 related to "residual" implies that lack of fit is insignificant, which means that the model fit the data well.

Table 10: Analysis of variance table for the Young's modulus of the MH series of the nanocomposites

\begin{tabular}{|lccccc|}
\hline Factors & DOF $^{\mathrm{a}}$ & $\begin{array}{c}\text { Sum of } \\
\text { squares }\end{array}$ & $\begin{array}{c}\text { Mean square } \\
\text { (Variance) }\end{array}$ & $\begin{array}{c}\text { F- } \\
\text { value }\end{array}$ & $\rho$-value \\
\hline Model & 13 & 7008.94 & 539.15 & 73.50 & $<0.0001$ \\
& & & & & \\
Concentration of & 2 & 2700.05 & 1350.02 & 184.0 & $<0.0001$ \\
PLLA (A) & 2 & 1798.11 & 899.06 & 4 & $<0.0001$ \\
Ratio of MCC/HA (B) & & & & 122.5 & \\
& & & & 7 & \\
Porogen content (C) & 1 & 2017.28 & 2017.28 & 275.0 & $<0.0001$ \\
AB & 4 & 253.72 & 63.43 & 1 & $<0.0001$ \\
ABC & 4 & 239.78 & 59.94 & 8.65 & $<0.0001$ \\
Residual & & & & 8.17 & \\
Lack of fit & 72 & 528.14 & 7.34 & 1.00 & 0.5000 \\
error & 36 & 264.07 & 7.34 & & \\
\hline
\end{tabular}

${ }^{\mathrm{a}}$ Degree of freedom

By comparing the F-values presented in Table 10, it is noticeable that the most influential factor (variable) onto Young's modulus response is the porogen content (factor C) because it has the highest F-value equal to 275.01; and then the concentration of PLLA (factor A) with a F-value of 184.04. The ratio of the MCC to HA (factor B) with a Fvalue of 122.57 has the effect less than $\mathrm{C}$ and A on the Young's modulus. Furthermore, Table 11 shows the statistics summary of the Young's modulus response of the MH series nanocomposites. 
Table 11: The summary of the statistics for Young's modulus response

\begin{tabular}{|ccccccc|}
\hline Response & STD & Mean & $\mathrm{R}^{2}$ & Adjusted R $^{2}$ & Predicted R & $\begin{array}{c}\text { Adequate } \\
\text { Precision }\end{array}$ \\
$\begin{array}{c}\text { Young's } \\
\text { modulus }\end{array}$ & 2.71 & 21.70 & 0.9299 & 0.9173 & 0.9180 & 27.676 \\
\hline
\end{tabular}

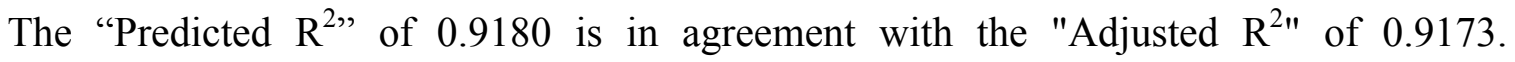
"Adequate precision" measures the signal to noise ratio. A ratio greater than 4 is desirable. Adequate precision ratio of 27.676 indicates an adequate signal, which means the model, can be used to navigate the design space.

\subsubsection{ANOVA analysis for mass loss response}

Based on the ANOVA of the mass loss response, factors (A, B, AC, $\mathrm{AB}, \mathrm{BC}$, and $\mathrm{ABC}$ ) represented the $p$-value less than 0.0001 . So, they affected the mass loss response significantly. On the other hand, factor (C) showed the $p$-value higher than 0.0500 at the ANOVA table, which indicated that they did not affect the mass loss of the MH series of nanocomposites significantly. So the factor $\mathrm{C}$ deleted from the model safely and the model corrected. After correction of the model with reduction of the insignificant factors, the final ANOVA table represented in Table 12. The "Model F-value" of 47.81 implies the model is significant and there is a $0.01 \%$ chance that this large F-value could occur due to noise. Also, the "lack of fit F-value" of 1.00 related to "residual" implies that Lack of fit is insignificant, which means that the model fit the data well. 
Table 12: ANOVA table for mass loss of the MH series of the MH series of nanocomposites

\begin{tabular}{|lccccc|}
\hline Factors & DOF & $\begin{array}{c}\text { Sum of } \\
\text { squares }\end{array}$ & $\begin{array}{c}\text { Mean square } \\
\text { (Variance) }\end{array}$ & F-value & $\rho$-value \\
\hline Model & 16 & 18715.57 & 1169.72 & 47.81 & $<0.0001$ \\
Concentration of & 2 & 852.50 & 426.25 & 17.42 & $<0.0001$ \\
$\begin{array}{l}\text { PLLA (A) } \\
\text { Ratio of MCC/HA (B) }\end{array}$ & 2 & 3632.49 & 1816.24 & 74.23 & $<0.0001$ \\
& & & & & \\
AB & 4 & 1929.66 & 482.42 & 19.72 & $<0.0001$ \\
AC & 2 & 4129.02 & 2064.51 & 84.38 & $<0.0001$ \\
BC & 2 & 1188.58 & 594.29 & 24.29 & $<0.0001$ \\
ABC & 4 & 6983.32 & 1745.83 & 71.35 & $<0.0001$ \\
& & & & & - \\
Residual & 36 & 880.86 & 24.47 & - & - \\
Lack of fit & & & 24.47 & 1.00 & \\
Pure error & & & 24.47 & & \\
& & & & &
\end{tabular}

By comparing the F-values presented in Table 12, it is noticeable that the most influential factor (variable) onto mass loss response is the ratio of the MCC to HA (factor B) because it has the highest F-value equal to 74.23. As mentioned earlier, the porogen content (factor C) has insignificant effect onto the mass loss response, so reduced in corrected model. Table 13 shows the statistics summary of the mass loss response of the nanocomposites.

Table 13: Summary of the statistics for the mass loss response

\begin{tabular}{|lcccccc|}
\hline Response & STD & Mean & $\mathrm{R}^{2}$ & $\begin{array}{c}\text { Adjusted } \\
\mathrm{R}^{2}\end{array}$ & $\begin{array}{c}\text { Predicted } \\
\mathrm{R}^{2}\end{array}$ & $\begin{array}{c}\text { Adequate } \\
\mathrm{R}^{2}\end{array}$ \\
Mass loss & 4.95 & 22.15 & 0.9140 & 0.8948 & 0.9060 & 23.168 \\
\hline
\end{tabular}

The predicted $\mathrm{R}^{2}$ and the Adjusted- $\mathrm{R}^{2}$ should be within 0.20 of each other. The

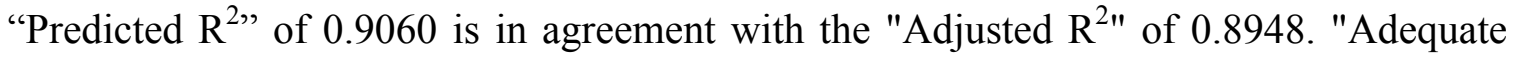


precision" measures the signal to noise ratio. A ratio greater than 4 is desirable. Adequate precision ratio of 23.168 indicates an adequate signal, which means the model, can be used to navigate the design space. The "predictive model" listed in coded terms. The coded (or pseudo) equation is useful for identifying the relative significance of the factors by comparing the factor coefficients. These equations of compressive yield, Young's modulus, and mass loss responses that used for predictions, in Appendix D.

\subsubsection{Optimization of conditions}

The main objective of design of experiments (DOE) was to create a model that will accurately model a process that can determine very precise optimum parameter setting. Then the success measured by analysis of variance (ANOVA). A significant $p$-value indicated an active factor and a reasonable estimate of its effects. The desirable high "adjusted" and "predicted R-squared" values (preferable $0.70^{+}$) was achieved. The Fvalue was used to show the contribution of each factor on the evaluated response. So, the next step is to optimize the composition of the $\mathrm{MH}$ series of nanocomposites, after obtaining the predictive equations represented (See Appendix.

Logistics of an experiment often make data collection more complicated. For example, because a certain medical device material synthesis needs a long process but the product launch date is fixed, there is a desire to reduce the number of experiments to be performed, while still being able to investigate many factors simultaneously. Rarely does a product have simply a single response (evaluated property). Optimizing a product with multiple responses is in many cases a trade-off issue. For example, in medical device properties such as compressive yield, the Young's modulus, resistance to water uptake, 
degradation rate, and crystallinity is important. In design of experiments (DOE) first goal was to identify the active factors (variables) and measure their effect on each response. In this study, the compressive yield, Young's modulus, and mass loss of the MH series of the nanocomposites were evaluated as response in analysis. By optimization of the parameters, it is possible to set the conditions for the parameters to obtain final responses with pre-identified value or maximum or minimum values. In order to maximize the compressive properties and minimize the water absorption or the mass loss od MH series of nanocomposites, the optimization utilized after analyzing with ANOVA. The Figure 14 shows the desirability values at each corner of the cube. Desirability values are ranged from 0 to 1.
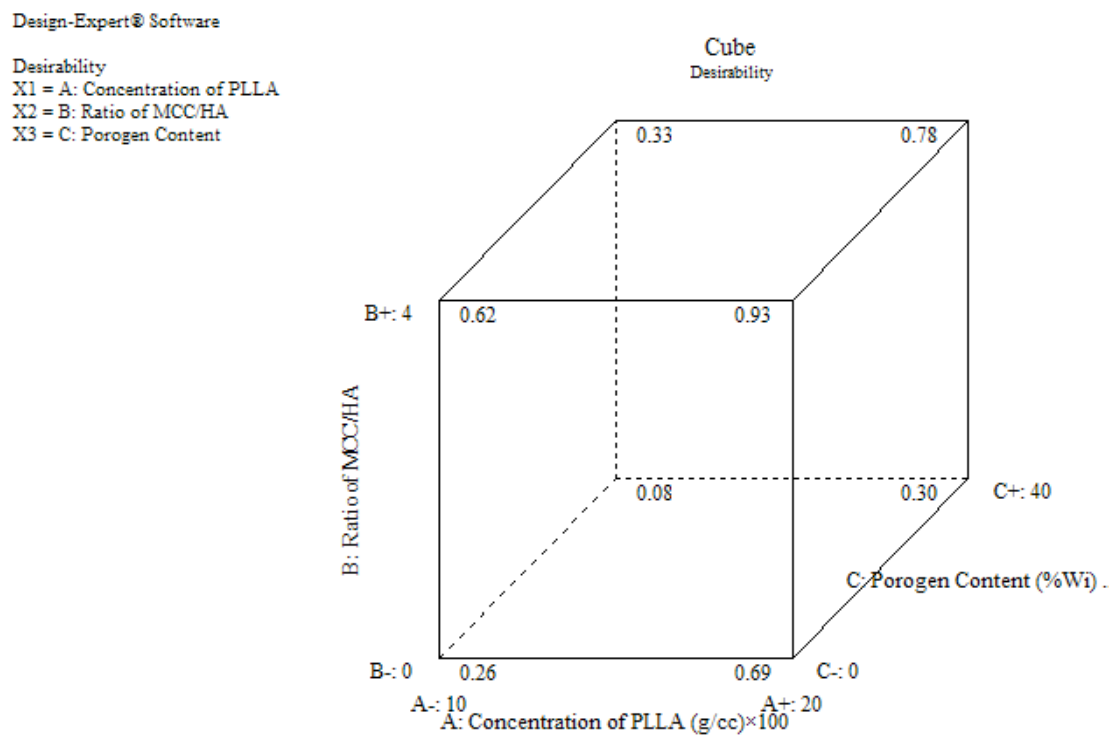

Figure 14: The desirability values for optimization of the parameters to obtain maximum compressive properties and minimum mass loss of the MH series of the nanocomposites

It can be seen that the highest desirability value is equal to 0.93 , which has the condition for parameters, the corner of the cubic on top-right of the cubic (Figure 14). This corner 
with highest desirability represents a nanocomposite with concentration of PLLA (factor A) equal to $20 \%$, the ratio of $\mathrm{MCC}$ to $\mathrm{HA}$ (factor B) equal to 4 , and the porogen content equal to 0 . Based on the Table 6, this specific nanocomposite, named MH $204 \mathrm{~N}$. By referring to Table 6 represented in section (3.2.3), it can be seen that values are related to MH $204 \mathrm{~N}$ nanocomposites.
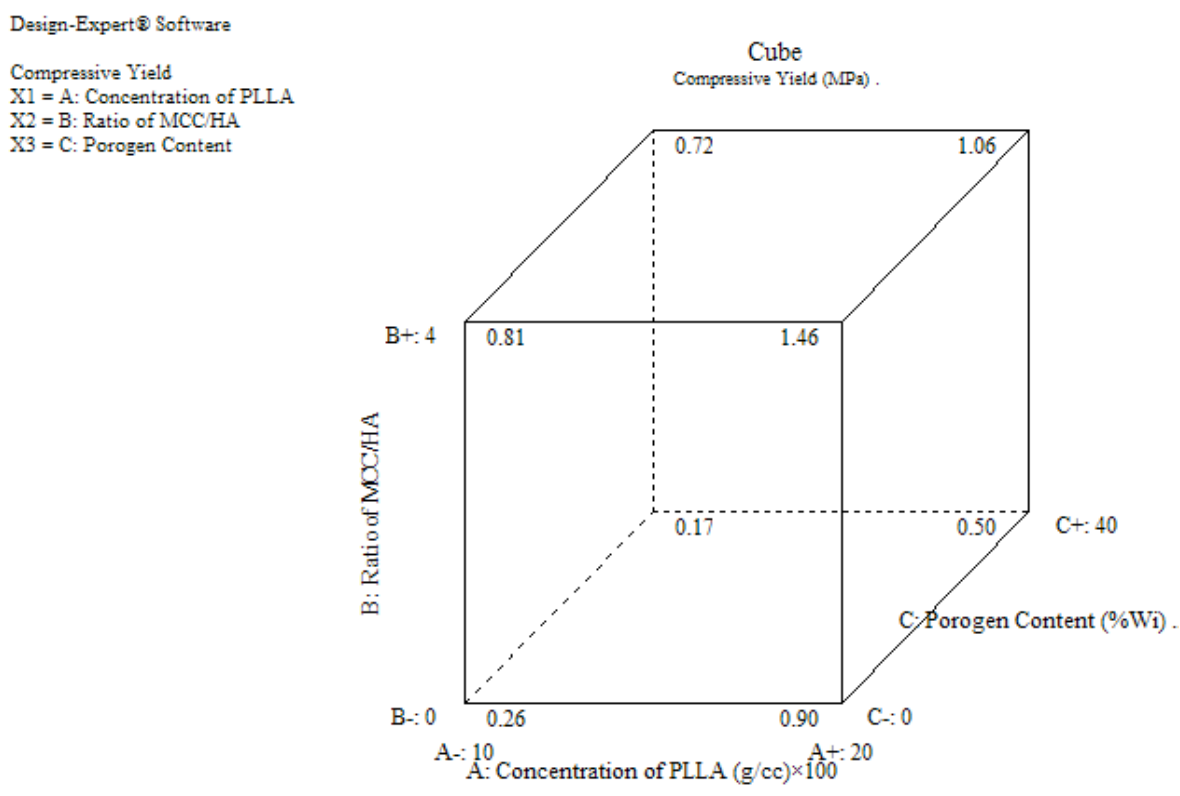

Figure 15: The compressive yield values for optimization of the parameters to obtain maximum compressive properties and minimum mass loss of the MH series of the nanocomposites

The predicted compressive yield value for this nanocomposites can be found from Figure 15 , which is equal to 1.46 MPa. The predicted Young's modulus value for the same nanocomposite, extracted from Figure 16 is $43.24 \mathrm{MPa}$, and the predicted mass loss value for the same nanocomposite is $4.45 \%$. 

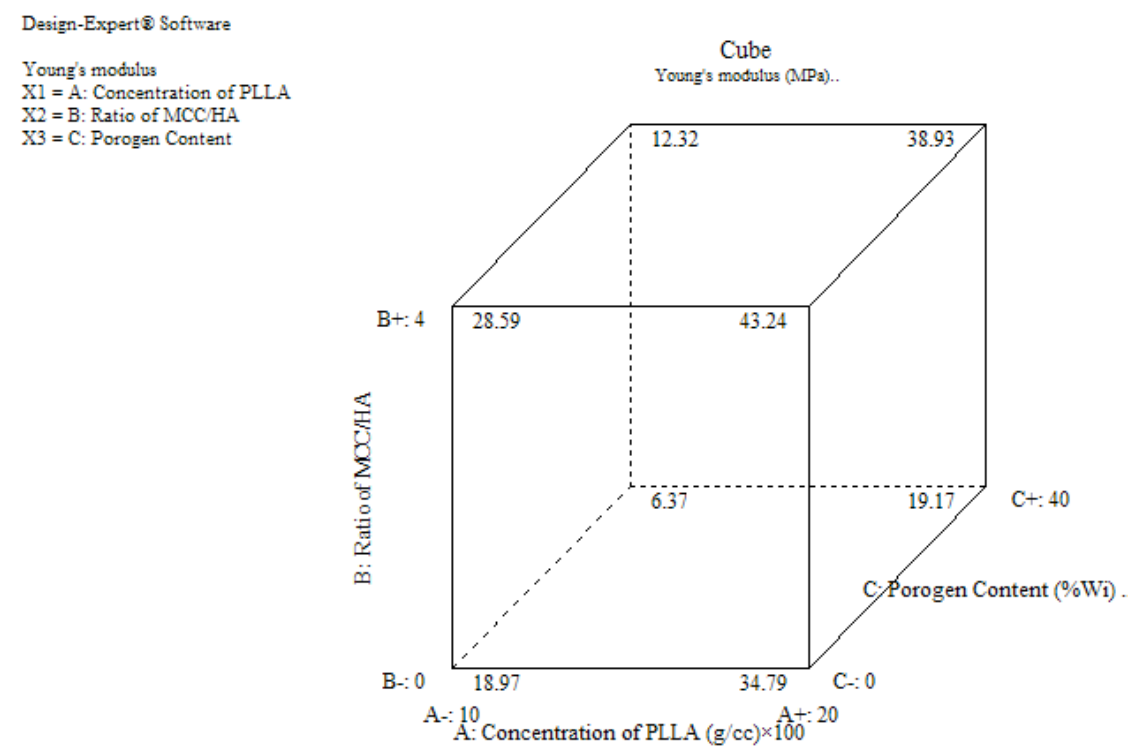

Figure 16: The Young's modulus values for optimization of the parameters to obtain maximum compressive properties and minimum mass loss of the MH series of the nanocomposites

After optimization of the parameters to achieve nanocomposites with desired responses, the mass loss of the MH series of nanocomposites can be extracted from Figure 17. This figure shows that by increasing the concentration of the PLLA from 10 to 20 (from left to right), the mass loss of the $\mathrm{MH}$ series of the nanocomposites, dropped from 33.95 to $11.75 \%$. This may be related to increasing the number of PLLA chains that would affect the overall strength of the nanocomposite by providing more mechanical strength and more potential sites for chemical bonding with HA nanoparticles and cellulose crystals via coupling agent (Felfel et al. 2013; Ward and Sweeney 2004). It can be seen that the amount of mass loss dropped when the concentration of the PLLA increased in Figure 17. 
Design-Expert 9 Software

Mass loss

$\mathrm{Xl}=\mathrm{A}$ : Concentration of PLLA

$\mathrm{X} 2$ = B: Ratio of $\mathrm{MCC} / \mathrm{HA}$

$\mathrm{X} 3=\mathrm{C}:$ Porogen Content

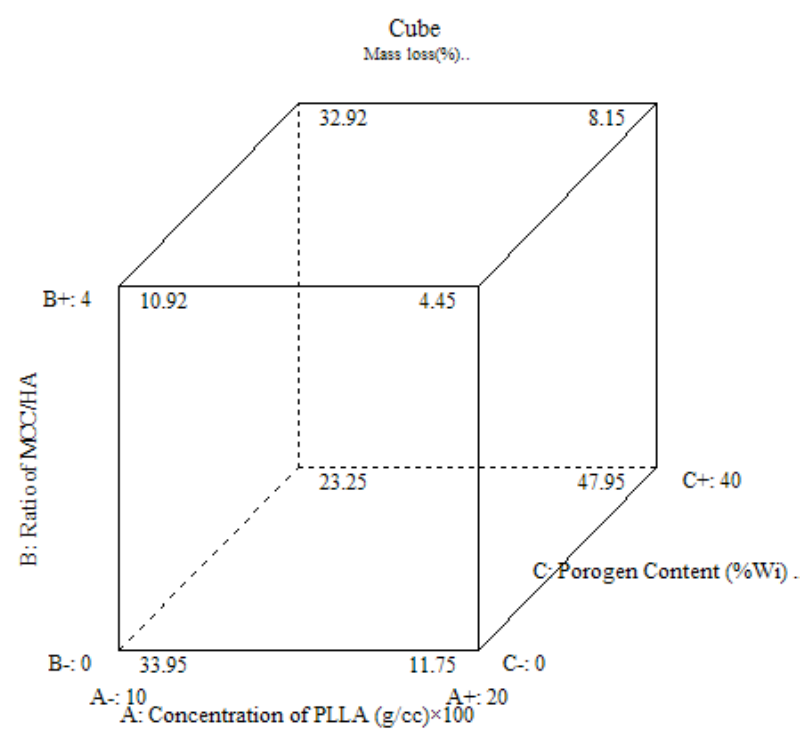

Figure 17: The mass loss values for optimization of the parameters to obtain maximum compressive properties and minimum mass loss of the MH series of the nanocomposites

Respectively, by increasing the ratio of cellulose over hydroxyapatite from 0 to 4 from bottom to up at left corner of Figure 17, the amount of mass loss decreased from 33.95 to $10.92 \%$. Based on the mechanical testing results, increasing concentration of PLLA from 10 to 20 , the compressive yield of the nanocomposites increased from 0.26 to $0.90 \mathrm{MPa}$, and their Young's modulus enhanced from 18.97 to $34.79 \mathrm{MPa}$, respectively. These results were extracted from Figures 15 and 16.

Besides, when the ratio of the cellulose over hydroxyapatite increased from 0 to 4 not only the mechanical strength of the nanocomposites improved, but also their mass loss amount decreased as well according to Figures 15, 16, and 17. All these improvements in mass loss of the $\mathrm{MH}$ series of the nanocomposites without sacrificing the mechanical properties by increasing the cellulose content is due to strong bonds between the PLLA 
matrix and reinforcing agents ( $\mathrm{MH}$ and $\mathrm{HA})$. All these strong bonds will decrease the amount of absorbed water into the structure of $\mathrm{MH}$ series of nanocomposites and subsequently, decreased their mass loss. On the other hand, the nanocomposites with higher content of cellulose (MH $204 \mathrm{~N}$ ), not only have higher values of compressive yield and Young's modulus, but also they have the lowest mass loss amount. It seems that what really affects the water absorption behaviour of the nanocomposites is the type of the constituents, their content or ratio and the strength of the bonding with the polymeric matrix and other constituents, which is the result of the compatibilization between the reinforcing agents including cellulose and the polymeric matrix.

After optimization of the conditions to obtain desired composition of nanocomposites, with favourable and pre-defined properties (responses), it is preferable to study the influence of the most influential factors onto the responses to understand the rationale and science behind them very well.

\subsubsection{Influence of the porosity of the MH series of the nanocomposites onto the shape of their stress-strain curves}

The porosity measurement results by liquid displacement method, revealed that the porosity of the $\mathrm{MH}$ series of the nanocomposites increased from 30 to $55-65 \%$ when the content of porogen increased from 0 to $40 \%$ weight fraction of the MCC and HA or 28\%

of the total weight of the MH series of nanocomposites. In Figure 18, the pink, red, and orange curves represent the nanocomposites specimens fabricated without using porogen and the dashed-line curves represent those with porosity. 


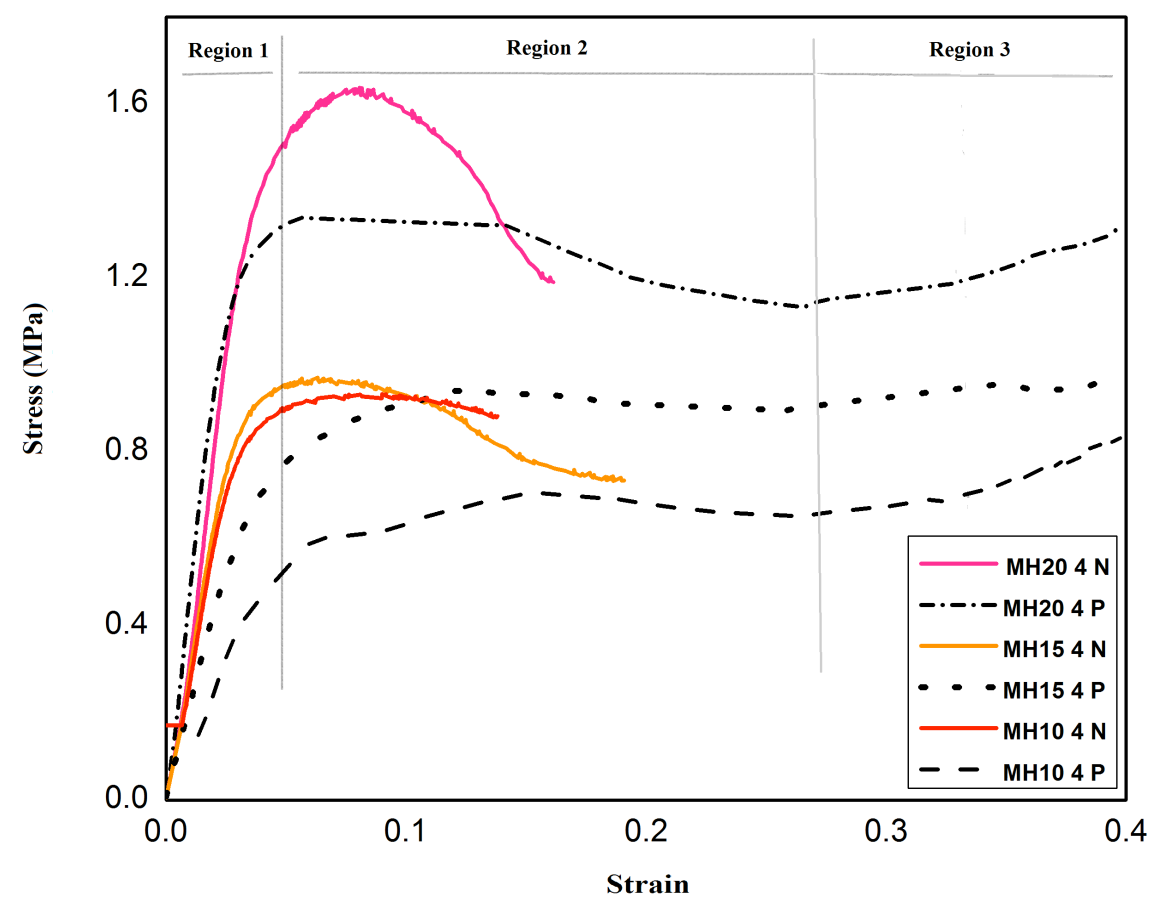

Figure 18: Typical stress-strain curves of the nanocomposites with identical ratio of $\mathrm{MCC} / \mathrm{HA}=4$ and various concentrations of PLLA and porogen content

All the nanocomposites had the same ratio of cellulose to hydroxyapatite, but different PLLA content (concentration of PLLA). In order to investigate the effect of porogen onto mechanical behaviour of the $\mathrm{MH}$ series of the nanocomposites, two pair of the nanocomposites with identical composition but a different porosity, were chosen. For instance, the pink curve belongs to a nanocomposite that has 30\% porosity (MH $204 \mathrm{~N}$ ) and the dashed curve right below that belongs to a nanocomposite with $55 \%$ porosity due to utilization of the porogen in its fabrication process (MH $204 \mathrm{P}$ ).

It can be seen that the different amount of porosity changed the behaviour of the stressstrain curve by decreasing the compressive yield and Young's modulus in dashed line curve in comparison with the pink curve. The values of the compressive yield stress and Young's modulus that extracted from the stress-strain curves showed that the 
compressive yield dropped over 13\% and Young's modulus dropped over $27 \%$ after the incorporation of the porogen to these nanocomposites with an identical composition. This is related to the effect of increasing porosity that lead to the weakening of the mechanical strength. The inverse effect of the porosity onto mechanical properties, also has been reported by others authors as well (Wolfang et al. 2007). The use of porogen has more significant influence on the Young's modulus than on the compressive yield stress. This conclusion can be drawn by the higher F-value of porogen content factor in comparison with other design parameters from ANOVA statistical analysis in Table 10 represented in section 4.2.2.

Besides, the influence of the utilization of porogen and increasing the porosity on the shape of the stress-strain curve is distinguishable by appearing a large deformation region in figure 18. This large strain deformation region is identified as region 2 and region 3 in the dashed-line curves, which are representing nanocomposites fabricated by porogen. In region 2, nanocomposites with higher porosity (dashed-line curves) showed extended ductility in their stress-strain curve. This behaviour can be attributed to the movement of the shear-banded zones in more porous specimens. So, further loading after the yield point, caused the shear-banded zone expansion till it consumes the entire sample (Schrauwen et al. 2004). This phenomenon may also be related to the collapse of pores under loading in more porous materials. This behaviour is observed in compression tests of porous specimens because the stress in compression suppresses the crack of propagation responsible for brittle failure and allows the material to behave plastically (Zedra et al. 2001). 
In region 1, the nanocomposites with a crystalline phase of the semi-crystalline PLLA obey the Hookean relationship, which means that the stress is proportional to strain before the yield point. After the yield occurs, the crystallites of PLLA do not form physical junction points, and the chain transports through the crystals (Piokowska et al. 2013).

Region 3 is a strain hardening phenomenon that seems to be related to "entanglement density", which is expected to be caused by reeling in the molecular chains of the PLLA polymer during the slow crystallization process of them (Ward et al. 2004) For semicrystalline thermoplastic polymers such as PLLA there is a direct relationship between entanglement density and strain hardening (Piokowaska et al. 2013).

The SEM images at Figure 19-a represents the structure of the MH $204 \mathrm{~N}$ (pink curve) nanocomposites with $30 \%$ porosity and figure $19-\mathrm{b}$ represents the porous structure of the MH $204 \mathrm{P}$ (dashed curve below the pink curve) with 55\% porocity. The influence of the utilization of the porogen is clear in terms of morphology of pores and their interconnectivity. Some particles of the porogen (salt) are still unwashed in Figure 19-b. Comparing the Figure 19-a with figure19-b showed that however the interconnectivity and shape of the pores of the MH series of the nanocomposites improved in comparison with MA series of the nanocomposites after incorporation of the porogen into their fabrication process, but still need further improvement. 


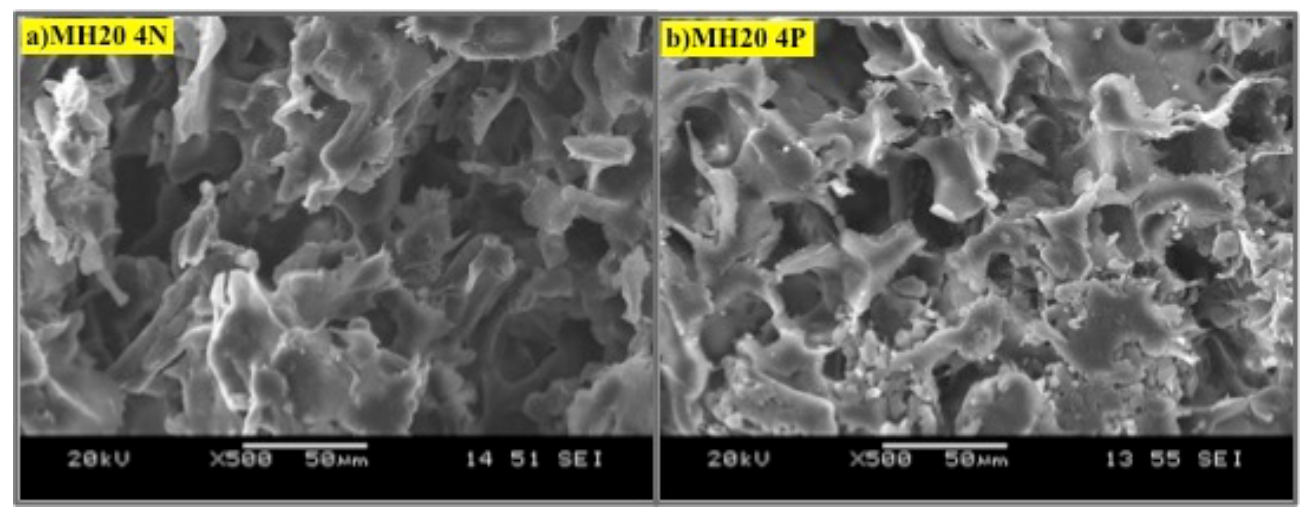

Figure 19: SEM images of nanocomposites, (a) MH 204 N, (b) MH 204 P

\subsubsection{Investigation of the effect of the content of the cellulose and hydroxyapatite of the MH series of the nanocomposites onto the shape of the stress-strain curves}

Nanocomposites with $0 \mathrm{~W}_{\mathrm{t}} \%$ of $\mathrm{MCC}$ (ratio of $\mathrm{MCC} / \mathrm{HA}=0$ ) were investigated separately. The values that extracted from the typical stress-strain curves of the $\mathrm{MH}$ series of these nanocomposites showed improved mechanical properties in comparison with the values found in the literature for composites made up of PLLA and hydroxyapatite. The typical stress-strain curves represented in Figure 20 demonstrates the influence of the increasing of the weight fraction of HA and increasing the concentration of PLLA onto the shape of their corresponding stress-strain curve.

For instance, the curve on top depicted in Figure 20, is related to $\mathrm{MH} 200 \mathrm{~N}$ nanocomposites, had a compressive yield of $0.90 \mathrm{MPa}$, and Young's modulus of 34.79 MPa. In the same Figure (Figure 20), the curve at the bottom is related to the MH $100 \mathrm{~V}$ nanocomposite. The compressive yield of that is equal to $0.26 \mathrm{MPa}$, and Young's modulus of $18.97 \mathrm{MPa}$. It can be easily understood that the increasing the concentration 
of PLLA from 10 to $20 \%$ in the nanocomposite reinforced with HA-solely from bottom to top in Figure 20, can improve the mechanical properties drastically. This can be related to increasing of the concentration of the PLLA and polymer chains in the nanocomposites, which provides more functional groups for chemical bonding with HA in presence of SDS as coupling agent (Schrauwen et al. 2004).

Also, it can be seen from Figure 20 that the nanocomposite with the lowest content of HA (28 $\mathrm{W}_{\mathrm{t}} \%$ ) has the highest mechanical strength. By increasing the content of HA nanoparticles from 28 to $40 \mathrm{~W}_{\mathrm{t}} \%$, the mechanical properties of the nanocomposites dropped. This result is consistent with the observations reported by other researchers such as Rhim et al. (2009). They observed deterioration in mechanical properties by the addition of more than $5 \mathrm{~W}_{\mathrm{t}} \%$ of clay nanoparticles to the PLLA matrix; they attributed this decrease to the poor dispersion of nano ceramic particles and necessity of an optimum value for the weight fraction of the nanoparticles in the nanocomposites. The values higher than optimum value can deteriorate the mechanical properties of the $\mathrm{MH}$ nanocomposites. 


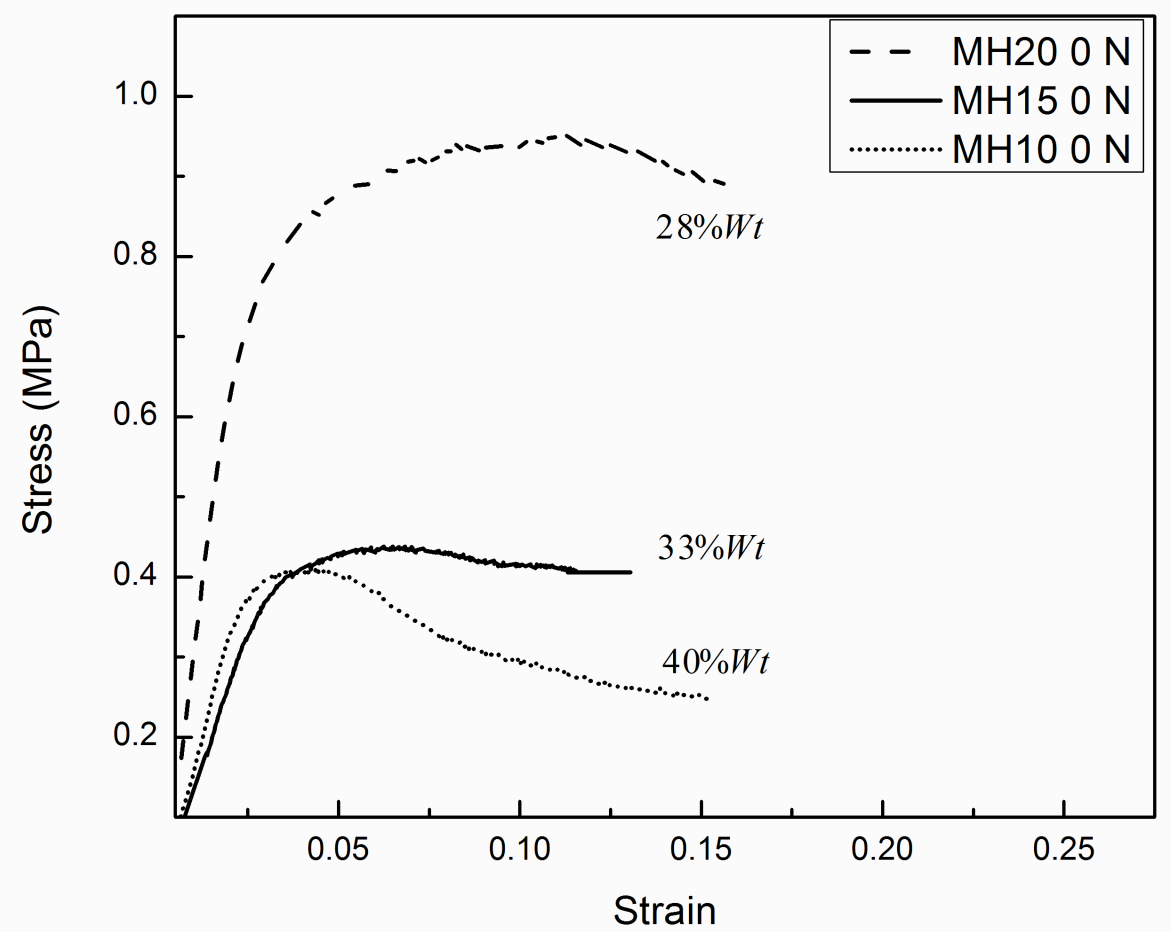

Figure 20: Typical stress-strain curve for the MH series of the nanocomposites reinforced by HA nanoparticles solely

Also, it can be seen from Figure 20 that the nanocomposite with the lowest content of HA $\left(28 \mathrm{~W}_{\mathrm{t}} \%\right.$ ) has the highest mechanical strength. By increasing the content of HA nanoparticles from 28 to $40 \mathrm{~W}_{\mathrm{t}} \%$, the mechanical properties of the nanocomposites dropped. This result is consistent with the observations reported by other researchers such as Rhim et al. (2009). They observed deterioration in mechanical properties by the addition of more than $5 \mathrm{~W}_{\mathrm{t}} \%$ of clay nanoparticles to the PLLA matrix; they attributed this decrease to the poor dispersion of nano ceramic particles and necessity of an optimum value for the weight fraction of the nanoparticles in the nanocomposites. The values higher than optimum value can deteriorate the mechanical properties of the $\mathrm{MH}$ nanocomposites. 
Also, it is clear from Figure 20, by decreasing the content of HA nanoparticles from 40 to $28 \mathrm{~W}_{\mathrm{t}} \%$ in comparison with nanocomposites with higher content of HA (from bottom to up), the area under the stress-strain curve increased. The area under the stress-strain curve represents the absorbed energy of the specimen before break. Since the HA is a rigid and brittle bioceramic, the higher amount of HA will result in a brittle fracture and lower absorbed energy before break. Besides, higher content of HA nanoparticles and their accumulation on the pores wall as shown in Figure (21-a), increases the chance of the agglomeration of the nanoparticles and deterioration of the mechanical properties of these nanocomposites. So, there is an optimum weight fraction of HA nanoparticles to obtain a nanocomposite with improved mechanical properties. The SEM images demonstrate the nanocomposites in which the weight fraction of HA nanoparticles increased from right to left (from $28 \mathrm{~W}_{\mathrm{t}} \%$ to $40 \mathrm{~W}_{\mathrm{t}} \%$ ). In Figure 21 However, no distinguishable change can be identified from these pictures in terms of pores structures.

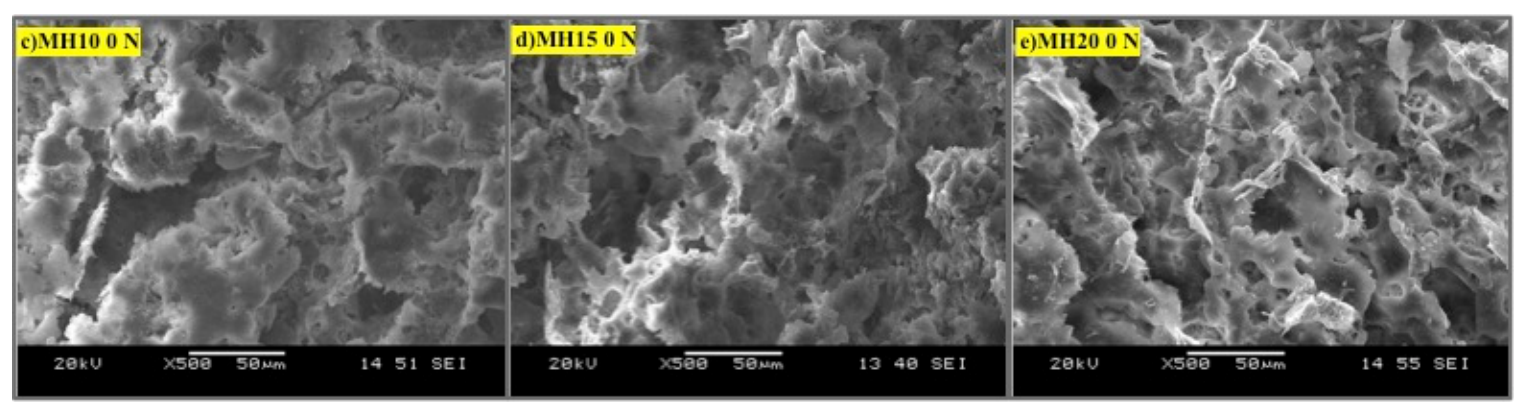

Figure 21: SEM images of nanocomposites MH 204 P, (c) MH 100 N, (d) MH 150 N, (e) MH $200 \mathrm{~N}$

While performing compression testing, we observed that the fracture occurred in a very brittle manner through the initiation and propagation of the oblique sharp cracks from the 
exterior corners of the specimen wall; Schrauwen et al. (2004) experienced the same observation while testing their PLLA-based nanocomposites reinforced with clay bioceramic nanoparticles. Our observations are consistent with other researchers' observations (Nieddu et al. 2009; Lewitus et al. 2006; Li et al. 2009). The surface modification of the bioceramic nanoparticles such as clays and hydroxyapatite nanoparticles both increase the basal spacing of them, so the polymer can easily enter between those spaces. So, the coupling agent serves as a compatibilizer between the hydrophilic bioceramics (HA or clays) and the hydrophobic polymer. By modifying the surface of the cellulose microcrystals and hydroxyapatite nanoparticles through the use of a coupling agent (SDS), a higher aspect ratio of nanoparticles can be incorporated into the polymer matrix (PLLA). Figure 22 shows that by increasing the ratio of MCC/HA from 0 to 4 , the compressive yield of the nanocomposites increased from 0.79 to 1.35 MPa for nanocomposites with no porogen involved in the fabrication procedure. This improvement is related to the reinforcing effect of MCC, which amplifies the reinforcing effect of HA nanoparticles. 


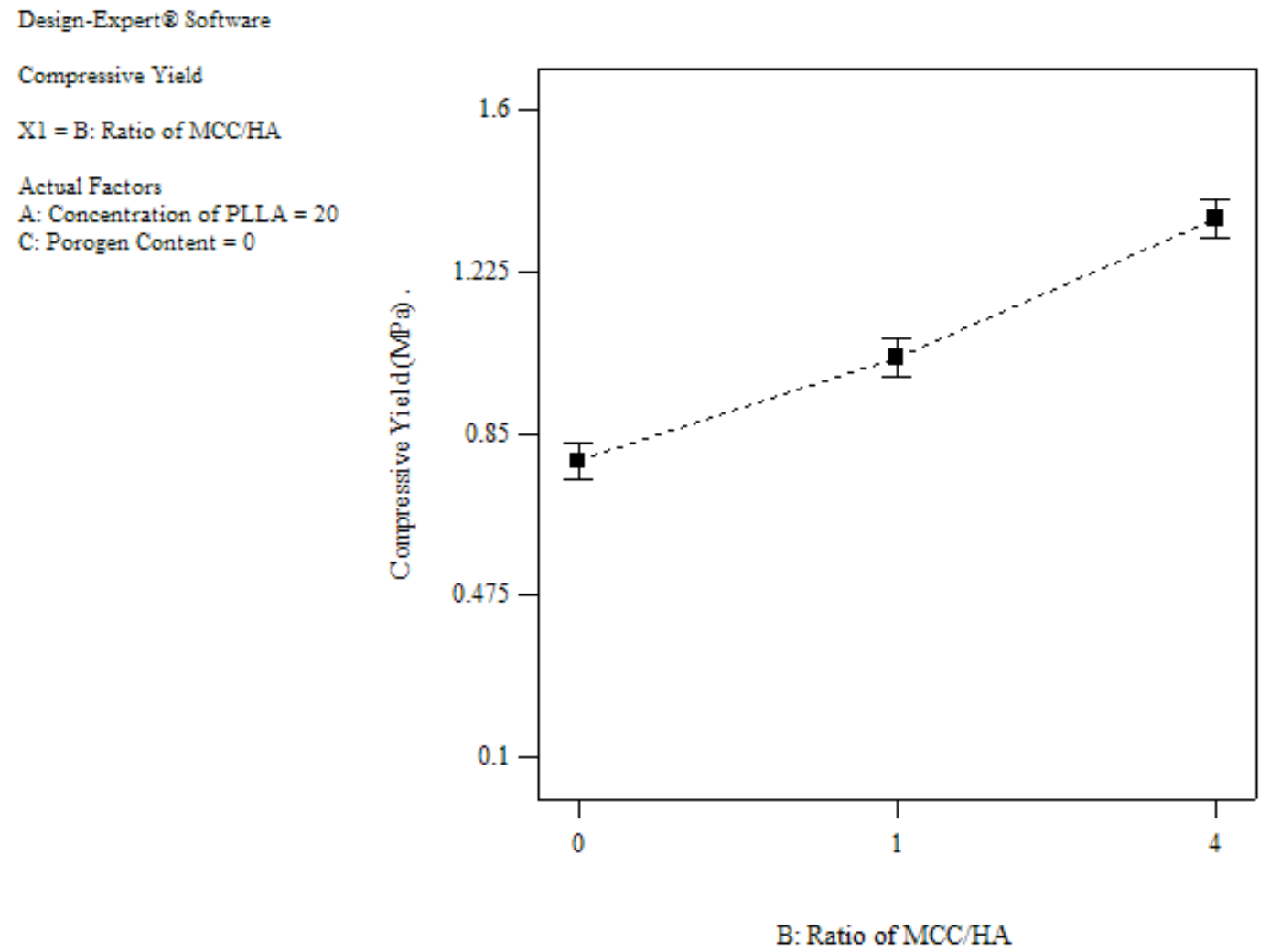

Figure 22: Compressive yield versus ratio of the $\mathrm{MCC} / \mathrm{HA}$ of the $\mathrm{MH}$ series of the nanocomposites with concentration of PLLA $(A=20)$, zero porogen content $(C=0)$

\subsubsection{The effect of operating variables on the water absorption behaviour and mass loss of the of the MH series of nanocomposites}

The water absorption behaviour of the biodegradable nanocomposites is important because it would affect the mass loss and biodegradation rate of those nanocomposites. The amount of absorbed water by the MH series of the nanocomposites was calculated by the weight difference between the samples immersed in water and the initial weight of the samples. The amount of absorbed water plotted against time for all the samples. The 
hydrophilic nature of the cellulose and hydroxyapatite nanoparticles was responsible for the water absorption of the MH series nanocomposites (Goetz \& Oksman, 2009).

For all tested nanocomposite samples, water absorption curves reached to plateau due to saturation after 72 hrs. Figure 23 compares the water absorption behaviour of three nanocomposites that have different amount of cellulose content in their composition, but identical other compositional parameters. So, the effect of the cellulose content on water absorption of the nanocomposites can be evaluated.

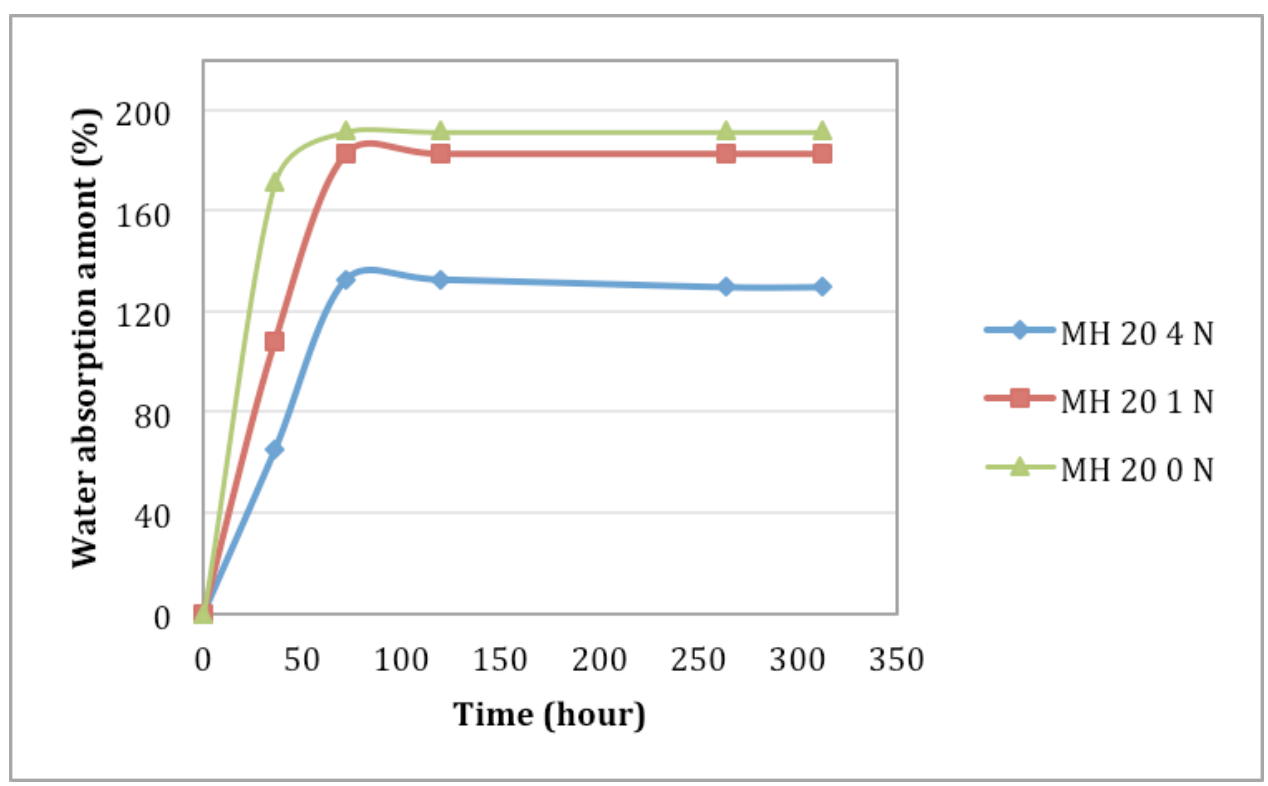

Figure 23: Water absorption curves of three MH series of the nanocomposites varying MCC to HA ratios, green curve, red curve, and the blue curve have the ratio of MCC to HA equal to $0,1,4$, respectively, the concentration of PLLA equal to $20 \%$ and porogen content equal to 0 for all three nanocomposites

Since MH $204 \mathrm{~N}$ nanocomposites (blue graph), have the highest ratio of MCC/HA and the highest content of the cellulose, it can be concluded that when the cellulose content is increased from 0 (blue curve) to 4 (for green curve), the saturation time is prolonged; the results showed that the improvement in water absorption in nanocomposites despite of 
the increase of the content of the cellulose. Since the absorbed water amount has a direct relationship with the degradation rate, the nanocomposites with the highest content of cellulose expected to show slower degradation rate in mass loss analysis results.

As can be observed from Figure 23, MH $200 \mathrm{~N}$ nanocomposites with zero content of cellulose (Ratio of $\mathrm{MCC} / \mathrm{HA}=0$ ) demonstrated the highest absorbed water amount, between $178-188 \%$; however, the nanocomposites with highest ratio of cellulose over hydroxyapatite (equal to 4) showed the least amount of absorbed water around $80 \%$, when saturated. These results are desirable but in disagreement with other authors findings (Espert et al. 2004). For instance, Espert et al. (2004) investigated the diffusion mechanisms of water absorption into polypropylene/natural cellulosic fibers composites. They observed that increasing the content of the natural fibers including cellulose in the composition of the composites would enhance their water absorption ability. They related that results to the higher porosity in the composites existence of voids that may accelerate the rate of diffusion of water into porous nanocomposites. However, based on the ANOVA results shown in section 4.2.1.3 for mass loss, it can have concluded easily that that porosity (porogen content) had no significant influence on the mass loss and water absorption amount, since by increasing the porosity by utilization of porogen, the amount of mass loss and absorbed water remained almost the same (the results of water absorption ANOVA has not been shown herein). So, we hypothesized that the increase of water absorption amount by increasing the content of the cellulose in composites fabricated by Espert et al. (2003), was not related to the porosity, it was due to the weak interfacial bonding of the natural fiber and polymeric matrix at composites and nanocomposites fabricated by their research team. By taking in to account that the $\mathrm{MH}$ 
series of nanocomposites became more resistant to water absorption even after increasing the content of the cellulose in their composition, it can be concluded that overall, all three mechanisms responsible for water absorption, as discussed earlier in literature review section were hindered the water absorption process. As depicted in Figure 24, the mass loss of the nanocomposites decreased when the ratio of the cellulose increased from 0 to 4 in nanocomposites with concentration of PLLA equal to $20 \%$ fabricated without porogen remained constant and there was no porogen involved in fabrication process. It is believed that the use of coupling agent improved the compatibility between cellulose crystals, hydroxyapatite nanoparticles, and PLLA matrix. Better compatibility between reinforcing agents and the PLLA matrix at an optimum ratio of cellulose over hydroxyapatite leads to even better adhesion of matrix to reinforcing agents. Furthermore, more hydrophilic groups such as hydroxyl groups of cellulose and hydroxy apatite are blocked after better adhesion with polymeric matrix because of bonding with matrix and coupling agent. Over all, better adhesion will disrupt the kinetics of diffusion process of the water molecules into the nanocomposites, and ultimately will decrease the velocity of diffusional process in nanocomposites with stronger interfacial bonding; this is also can be related to the elimination of the gaps at the interfacial regions. This is also another sign of the improved compatibility of cellulose microcrystals and fibers with the PLLA matrix interface. The improved water absorption resistance of the nanocomposites will decrease the mass loss and biodegradation rate of those nanocomposites. As we mentioned earlier in the objectives of the research, one of the important challenges in the case of cellulose containing nanocomposites is improving their water resistance when the content of cellulose increases (Zimmermann et al. 2011; Zadegan et al. 2011). 

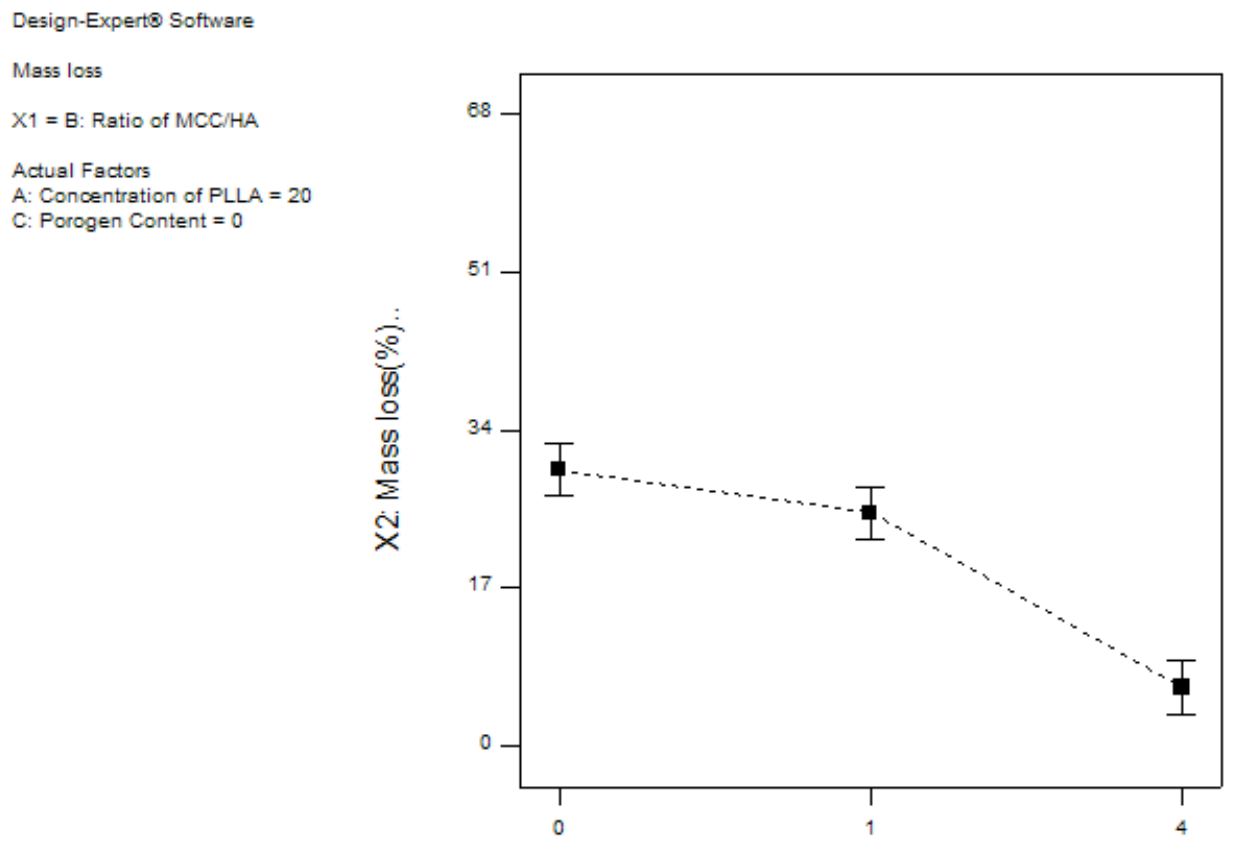

X1: B: Ratio of MCC/HA

Figure 24: The effect of ratio of MCC to HA (factor B) onto water absorption of the (MH 20 $0 \mathrm{~N}$ ), (MH $201 \mathrm{~N}$ ), and (MH $204 \mathrm{~N}$ ) nanocomposites which have various ratio of $\mathrm{MCC} / \mathrm{HA}$ equal to 0,1, and 4, but identical concentration of PLLA equal to 20 (factor $A$ ) and porogen content (factor $\mathrm{C}$ ) equal to 0

The MH $204 \mathrm{~N}$ series of nanocomposites with higher content of cellulose over hydroxyapatite (Ratio of $\mathrm{MCC} / \mathrm{HA}=4$ ) that showed the lowest water absorption percentage and the lowest mass loss amount as compared with the others showed the lowest mass loss after being immersed in water for biodegradation experiment for three months. 


\section{Chapter 5. CONCLUSIONS AND FUTURE WORK}

The main goal of this research was to develop a novel synthetic bone graft biomaterial that resembles biological, mechanical, chemical, and thermal properties of real bone. The main objective of this research was to develop biomimetic nanocomposites, which possess properties similar to autologous bone such as morphology, composition, microstructure, and mechanical properties. To meet the main goal, two stages involving development of the MA series and then $\mathrm{MH}$ series of the nanocomposites completed in two stages.

Stage 1: The MA series of the nanocomposites developed by varying the weight ratio of cellulose and hydroxyapatite over Poly L-Lactic acid to find an optimum range for the weight fraction of each constituent. The appropriate ranges for weight fraction of the constituents of the MA series of the nanocomposites were identified.

1. The dispersion of the cotton source microcrystals (MCC) and hydroxyapatite (HA) nanoparticles, improved by implication of chemical and mechanical methods such as incorporation of coupling agent and ultra-sonication. The yield suspension was a uniform colloidal mixture in which reinforcing agents dispersed homogenously in PLLA solution with no sign of agglomeration. This was related to better interfacial bonding of MCC and HA nanoparticles with matrix. The chemical reaction behind this phenomenon is that the hydrophilic head of SDS absorbed to the cellulose surface, whereas its hydrophobic tail attached to the PLLA. Besides, the SDS is able to bond to $\mathrm{Ca}^{2+}$ through ionic phosphate and carboxylate groups of the HA. 
2. The thermal properties of the MA series of the nanocomposites evaluated by DSC analysis. DSC analysis confirmed the increase of the crystallinity of the MA series of the nanocomposites by increasing the content of the MCC and HA in them. The crystallinity of PLLA increased up to $38 \%$ by increasing the content of MCC and HA from 0 to $21 \mathrm{~W}_{\mathrm{t}} \%$. The results show the effect of increasing the weight ratio of MCC and HA on the crystallinity of the nanocomposites, which may have caused by higher amount of ordered bonds via hydrogen bonding leading to an increase of the crystallinity.

3. The values of the compressive strength and modulus of the MA series of the nanocomposites, which extracted from the typical curves represented shows that the weight ratio of MCC over PLLA increased from 0.1 to 0.5 for MA1010 to MA5050. This led to an improvement in the compressive yield stress from 0.127 (Pristine PLLA) to 2.2 MPa (MA5050) and the Young's modulus from 6.6 MPa (Pristine PLLA) to $38 \mathrm{MPa}$ (MA5050), respectively. Increasing HA content of the composites may increase crystallinity of the nanocomposites due to the presence of ordered hydrogen bonding and the ceramic nature of the HA leading to higher mechanical properties. The increase of the weight ratio of MCC and HA to PLLA from 0 to 0.5 increased the compression yield stress by $90 \%$ and the Young's modulus of the nanocomposites by $82 \%$. This significant increase may be attributed to hydrogen bonding between the reinforcing agents (MCC and HA) and the PLLA matrix.

4. The morphology and microstructure of the MA series of the nanocomposites were examined using SEM. The pristine specimen seemed to be an internal porous 
structure. The SEM micrographs revealed that HA nanoparticles and MCC fibers are distributed in the matrix randomly and homogeneously, i.e. some are embedded in pore walls and some piled between pores and there was no sign of agglomeration. Some agglomeration in the MA6060 composite was observed. This agglomeration affected the mechanical properties of the composites and makes the composite more brittle.

5. The toxicity of the novel biomaterial evaluated in exposure to bone cells and MTT assay. Viability of the cells assessed by exposure of cells to the extract of three sets of the nanocomposites. Cells viability generally was not affected even after 24, 48 and $72 \mathrm{hrs}$. incubation time for all extracts compared to the control. For MA4040, and MA5050 nanocomposites, proliferation of the cells caused increasing the population of the cells.

Stage 2): The second series of nanocomposites named $\mathrm{MH}$ series, designed and developed to optimize the conditions for having maximum compressive properties and minimum the water absorption or the mass loss after analyzing with ANOVA.

6. The $\mathrm{MH}$ series of the nanocomposites by selecting the narrower range of the weight fraction of the constituents and incorporation of porogen designed and developed. The models that accurately modeled the process that used for precise optimum parameter setting. The active factors (variables) and measure their effect on each response identified. The simultaneous effect of MCC to HA ratio, concentration of PLLA, and porogen content onto the mechanical and water absorption, and biodegradation behaviour of the $\mathrm{MH}$ series of the nanocomposites evaluated. 
7. The mechanical properties and mass loss of the nanocomposites can be tailored to be used as biodegradable bone implant to match the properties of the surrounding bone at the damaged site.

8. The contribution of each factor on evaluated property (response) identified. Then the water absorption resistance of the MH series of the nanocomposites improved and then their biodegradation rate without scarifying their mechanical properties especially improved when the content of natural fiber was increased. Then the process parameters (variables) optimized in order to maximize the compressive properties and minimize the water absorption or the mass loss after analysis of variances by utilization of ANOVA and obtaining predictive equations.

9. Analysis of variance statistical analysis (ANOVA) showed that the ratio of cellulose over hydroxyapatite was the most influential factor affecting the compressive yield and the mass loss, while the porogen content was the most detrimental factor affecting the Young's modulus, had no significant effect on the rate of the mass loss of MH series of the nanocomposites. So, the optimization the process parameters performed in order to maximize the compressive properties and minimize the water absorption or the mass loss.

For future work, I would recommend the following list:

1. To test the biocompatibility and osteogenesis of the biomaterial in vitro, also test the proliferation of the cells when directly contacting to the nanocomposites surface to evaluate cell attachment as well and then perform animal testing in vivo 
2. In order to fabricate more custom fit implants, 3-D printing for fabrication of the final product looks promising.

3. 3D printing method can also fabricate more accurate pore sized, homogenous pore shape, uniform pore distribution, and controlled porosity.

4. Besides, the biologic biomolecules such as bone morphogenic proteins or drugs such as pain relievers can be immobilized onto the surface of this biomaterials due to presence of cellulose in their composition.

5. Utilization of cellulose nanofibers or functionalized carbon nanotubes instead of microcrystalline cellulose to develop new nanocomposites

6. Application of the $\mathrm{MH}$ nanocomposites for industrial purposes that requires improvement of the water absorption of the cellulose or improvement of the biodegradation rate of the poly (lactic acid)

7. 3D printing method can also fabricate more accurate pore sized, homogenous pore shape, uniform pore distribution, and controlled porosity.

8. Besides, the biologic biomolecules such as bone morphogenic proteins or drugs such as pain relievers can be immobilized onto the surface of this biomaterials due to presence of cellulose in their composition.

9. Finding industrial application for nanocomposites reinforced by cellulose in which cellulose fibers are isolated

10. Evaluate the homogeneity of the colloidal mixture by turbidity test and TEM.

11. Investigation of the correlation between the crystallinity of PLLA and mass loss of the $\mathrm{MH}$ nanocomposites 


\section{LIST OF APPENDICES}

Appendix A: DSC graphs used for crystallinity calculation

Appendix B: Image analysis results of MA series of nanocomposites

Appendix C: The picture of the micro-plates used for MTT toxicity assay

Appendix D: Final predictive equations in terms of coded factors 


\section{Appendix A.}

\section{DSC graphs used for crystallinity calculation}

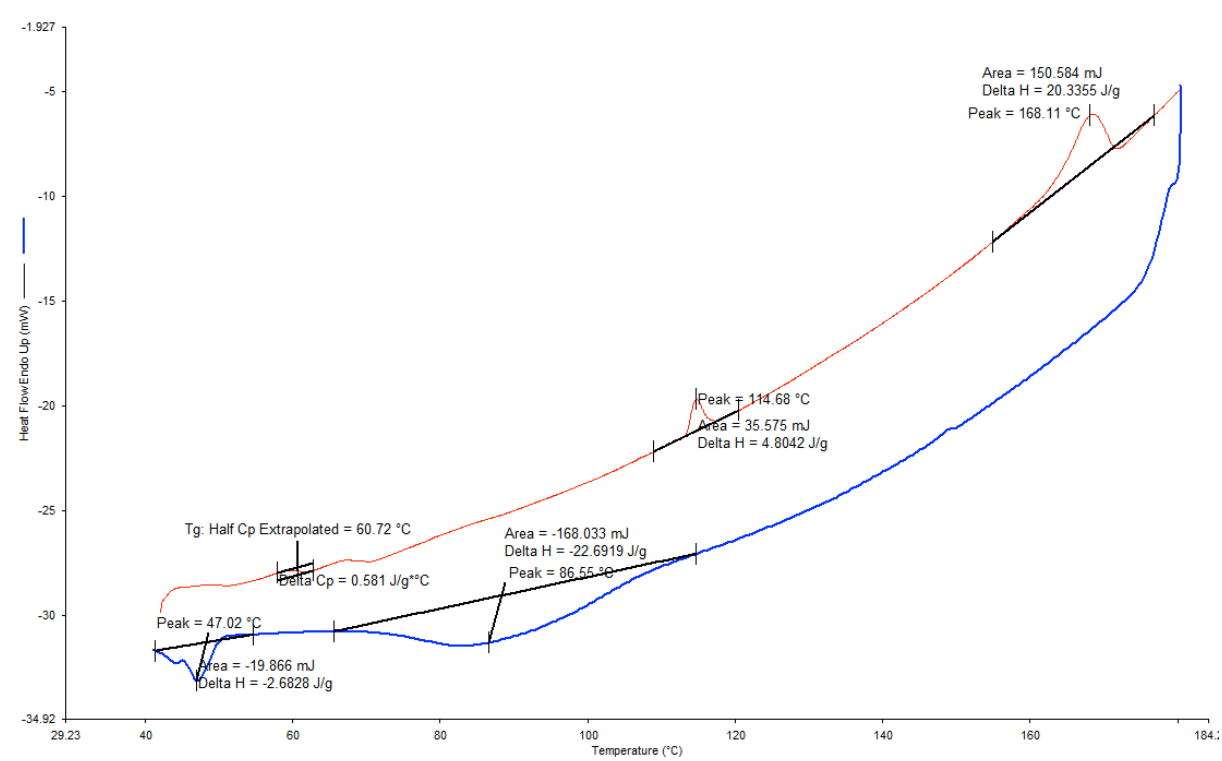

Figure A.1. The heating curve (red) and cooling curve (blue) graph of sample 5

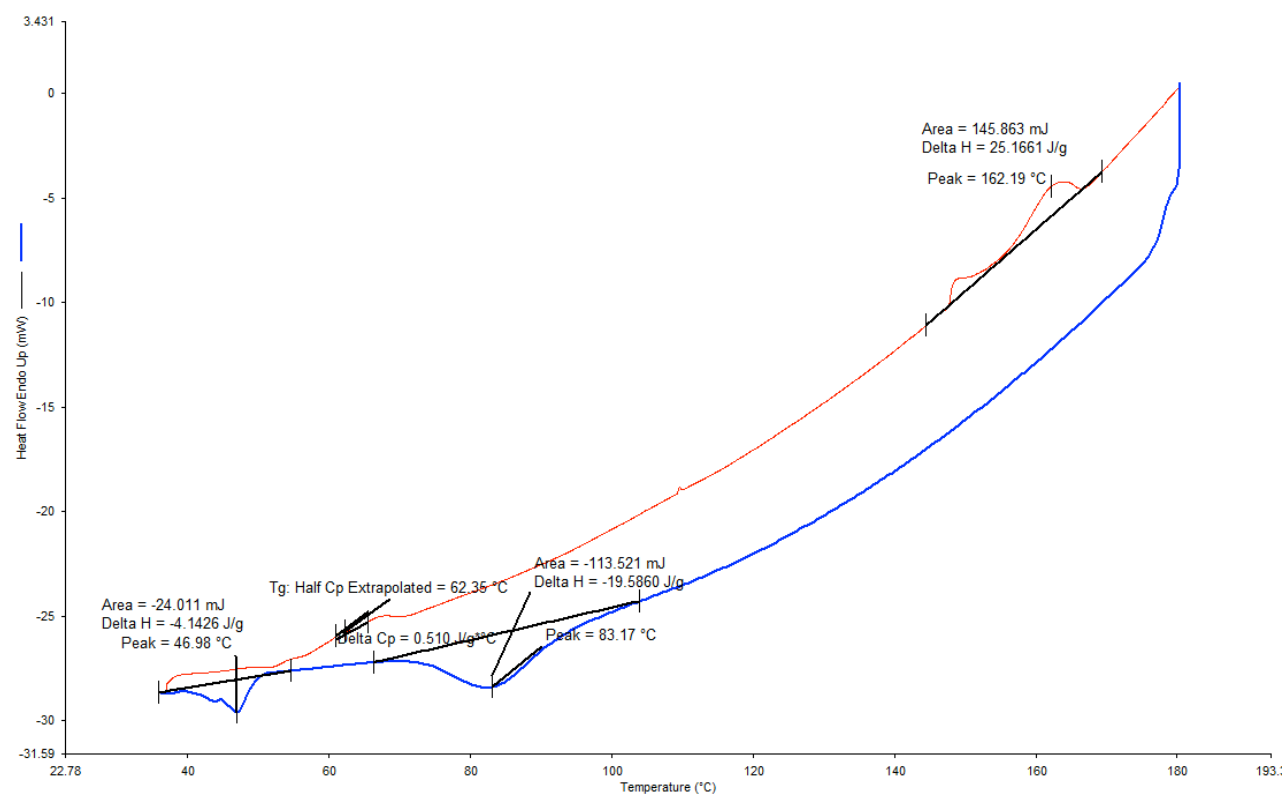

Figure A.2. The heating curve (red) and cooling curve (blue) graph of sample 20 


\section{Appendix B.}

\section{Image analysis results of MA series of nanocomposites}

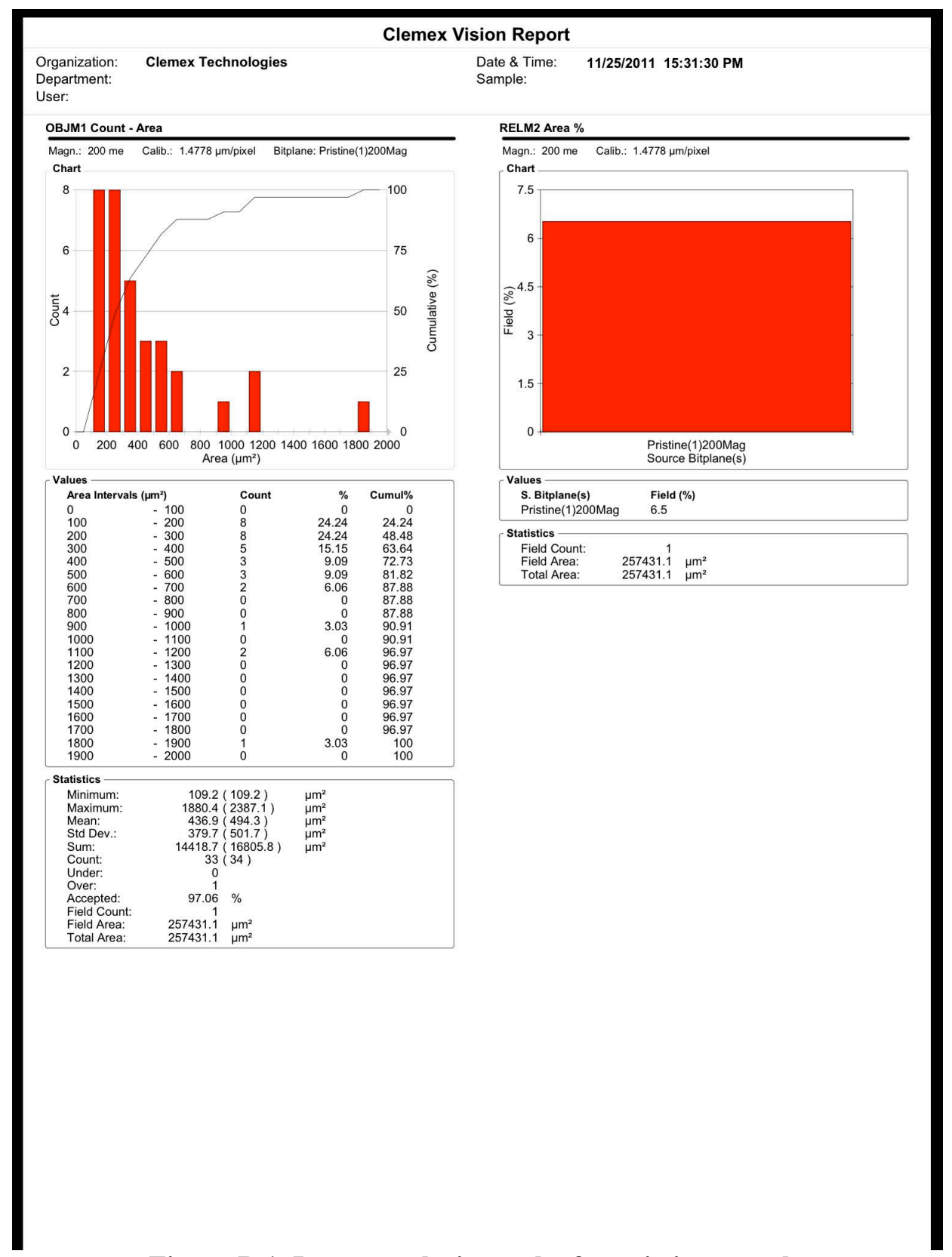

Figure B.1. Image analysis results for pristine sample 


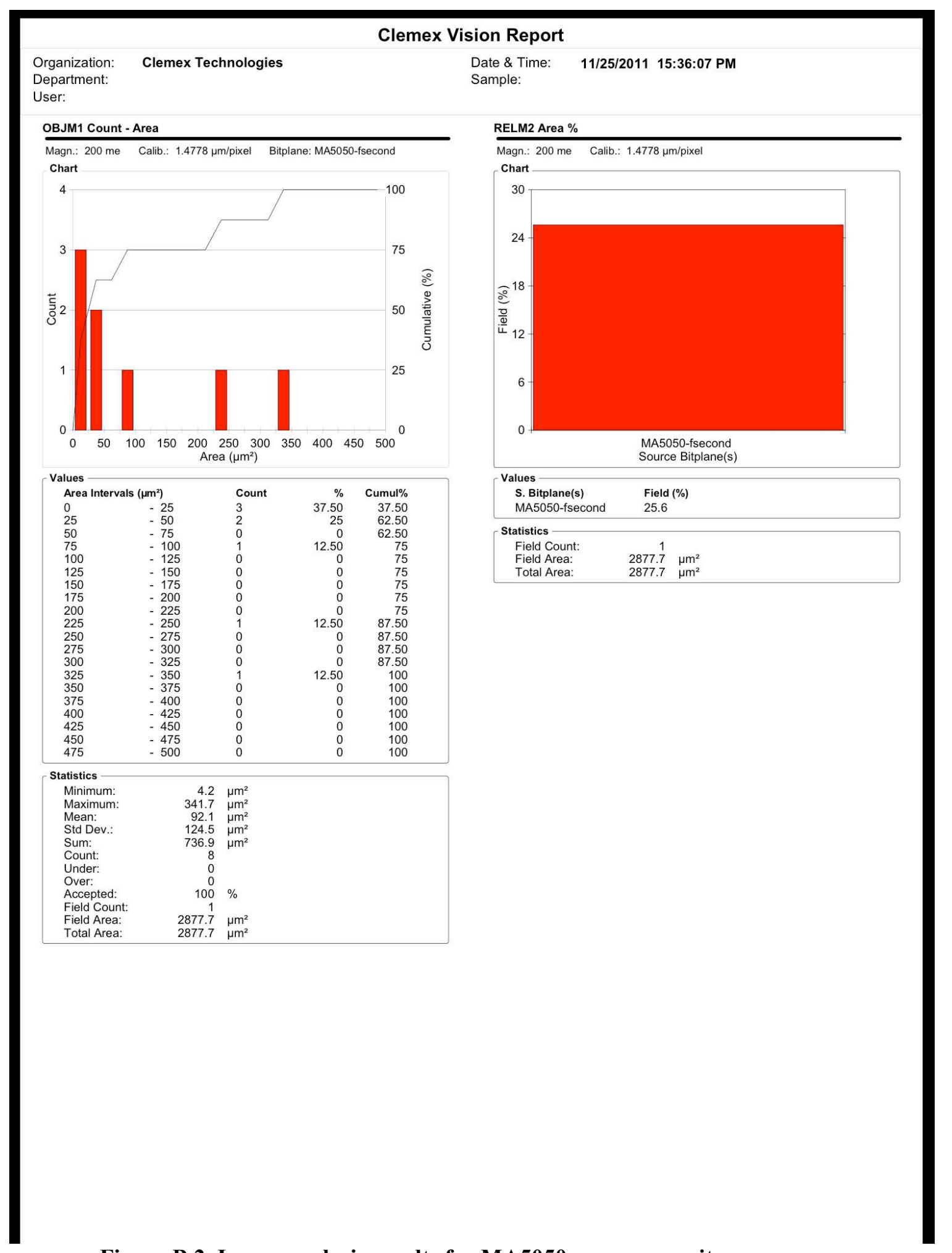

Figure B.2. Image analysis results for MA5050 nanocomposite 


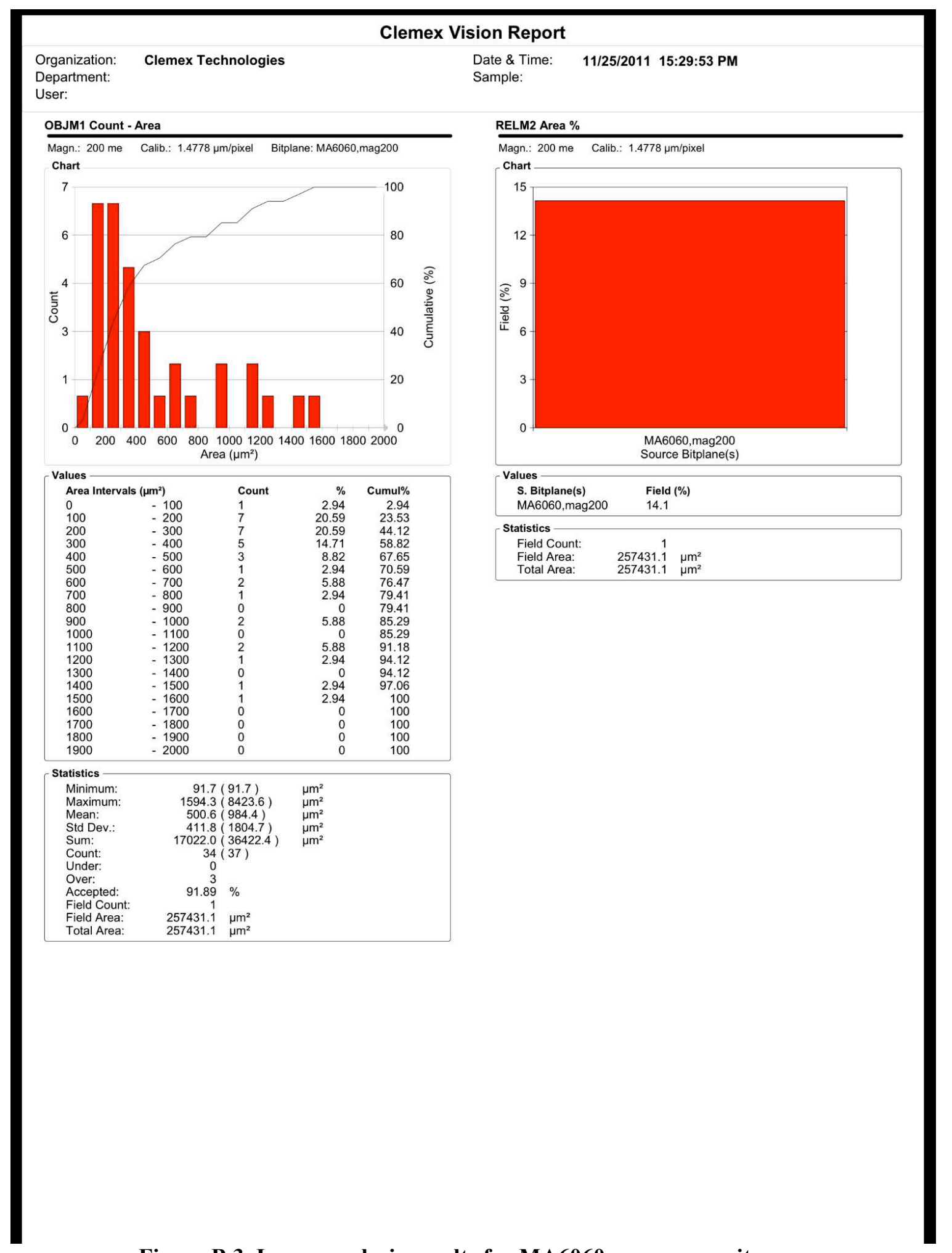

Figure B.3. Image analysis results for MA6060 nanocomposite 


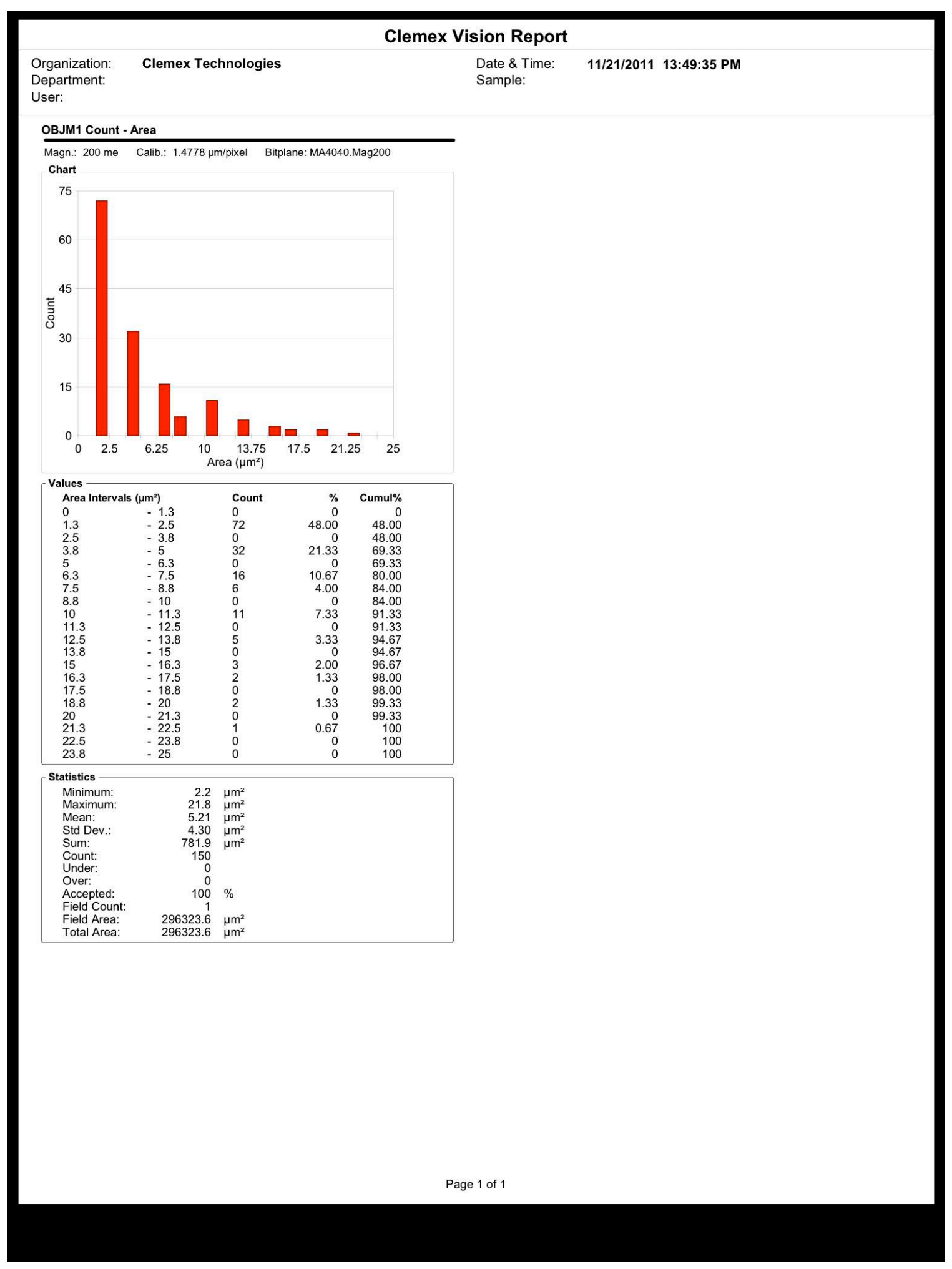

Figure B.4. Image analysis results of MA4040 nanocomposite 


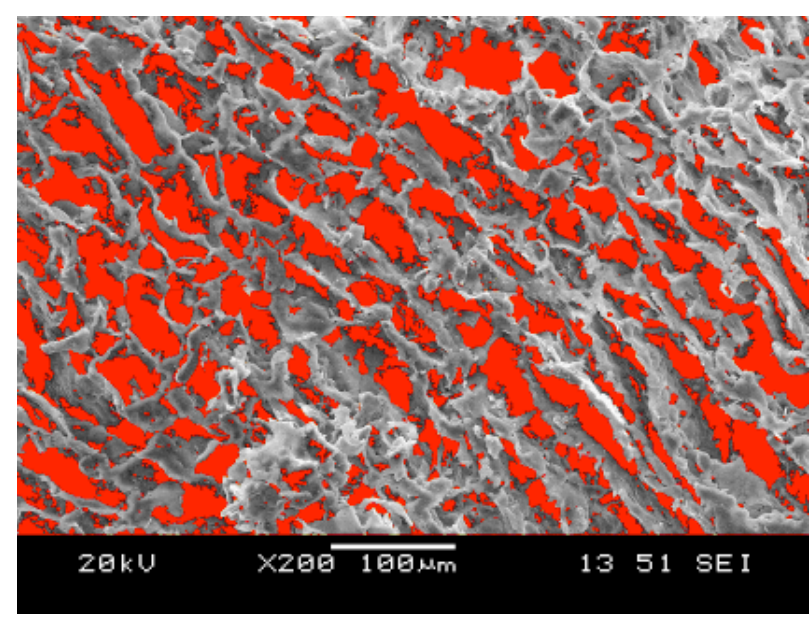

Figure B.5.The image that was used for the image analysis calculations 


\section{Appendix C.}

\section{The picture of the microplates used for MTT toxicity assay}

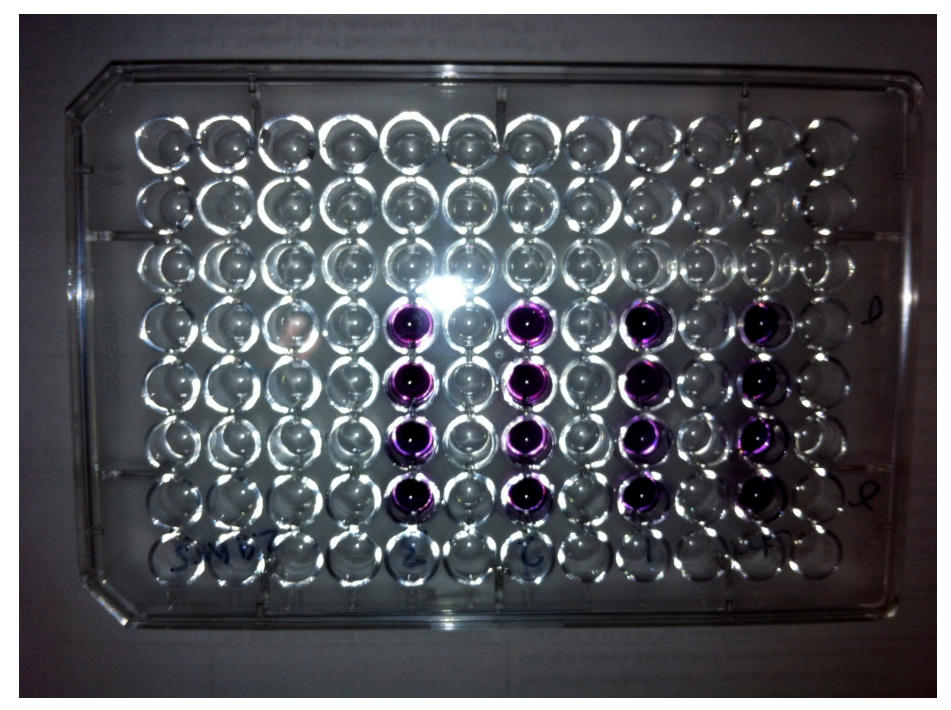

Figure C.1. The results of toxicity after $24 \mathrm{hrs}$ culture of cells in biomaterial extract medium

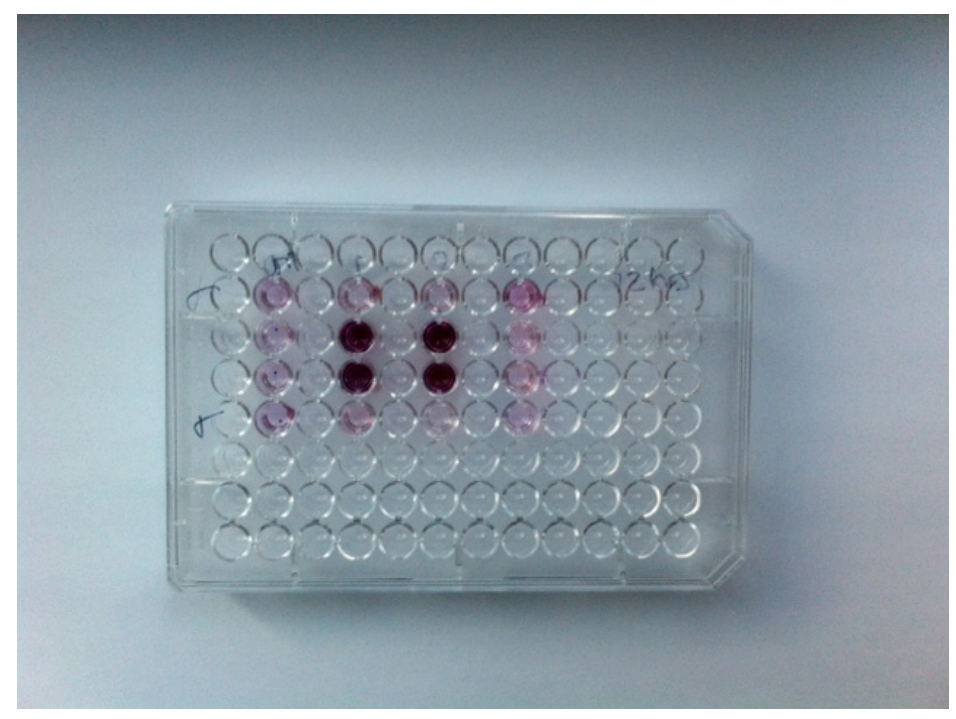

Figure C.2. The results of toxicity after $72 \mathrm{hrs}$ culture of cells in biomaterial extract medium 


\section{Appendix D.}

\section{Final predictive equations in terms of coded factors}

The "predictive model" listed in coded terms. The coded (or pseudo) equation is useful for identifying the relative significance of the factors by comparing the factor coefficients. These three equations that used for predictions.Final Equation in Terms of Coded Factors:

Equation D.1: Compressive Yield $=+0.71-0.23 \times \mathrm{A}[1]-0.028 \times \mathrm{A}[2]-0.27 \times \mathrm{B}[1]-0.028 \times$ $\mathrm{B}[2] 0.089 \times \mathrm{C}+0.044 \times \mathrm{A}[1] \mathrm{C}+0.068 \times \mathrm{A}[2] \mathrm{C}$

Equation D.2: Young's modulus $=+21.70-6.33 \times \mathrm{A}[1]-3.54 \times \mathrm{A}[2]-5.38 \times \mathrm{B}[1]-2.62 \times \mathrm{B}[2]$

$-6.11 \times \mathrm{C}+2.68 \times \mathrm{A}[1] \mathrm{B}[1] 3.47 \times \mathrm{A}[2] \mathrm{B}[1]+0.24 \times \mathrm{A}[1] \mathrm{B}[2]+2.06 \times \mathrm{A}[2] \mathrm{B}[2]-0.19$

$\times \mathrm{A}[1] \mathrm{B}[1] \mathrm{C}+1.88 \times \mathrm{A}[2] \mathrm{B}[1] \mathrm{C}+2.21 \times \mathrm{A}[1] \mathrm{B}[2] \mathrm{C}+0.050 \mathrm{~A}[2 \times] \mathrm{B}[2] \mathrm{C}$

Equation D.3: Mass loss $=+22.15+5.50 \times \mathrm{A}[1]-3.76 \times \mathrm{A}[2]+9.89 \times \mathrm{B}[1]+0.30 \times \mathrm{B}[2]-8.94 \times$ $\mathrm{A}[1] \mathrm{B}[1]+9.39 \times \mathrm{A}[2] \mathrm{B}[1]+4.48 \times \mathrm{A}[1] \mathrm{B}[2]-8.86 \times \mathrm{A}[2] \mathrm{B}[2]-4.19 \times \mathrm{A}[1] \mathrm{C}-7.98 \mathrm{~A}[2] \mathrm{C}-$ $5.97 \times \mathrm{B}[1] \mathrm{C}+0.49 \times \mathrm{B}[2] \mathrm{C}+4.81 \times \mathrm{A}[1] \mathrm{B}[1] \mathrm{C}-$

$16.71 \times \mathrm{A}[2] \mathrm{B}[1] \mathrm{C} 14.51 \times \mathrm{A}[1] \mathrm{B}[2] \mathrm{C}+10.60 \times \mathrm{A}[2] \mathrm{B}[2] \mathrm{C}$

Herein, the coefficient "A[1]" represents the linear component, and A[2] represents the quadratic component. 


\section{REFERENCES}

Agrawal C., and Ray R. (2001). Biodegradable polymeric scaffold for musculoskeletal tissue engineering. Journal of Biomedical Material Research, 55 (2), 141-150.

Amini A., Laurencin C., Nukavarapu S.P. (2012). Bone tissue engineering: Recent advances and challenges. Critical Review in Biomedical Engineering, 40 (5), 363-408.

Armentanoa I., Bitinis N., Fortunatia B.E., Mattiolia S., Rescignanoa N., Verdejob R., Lopez-Manchadob M.A., Kennya J.M. (2013). Multifunctional nanostructured PLA materials for packaging and tissue engineering. Progress in Polymer Science, 38, 17201747.

Asefnejad A., Khorasani M.T., Behnamghader A. and Khorasani.M.T. (2011) Polyurethane/fluor-hydroxyapatite nanocomposite scaffolds for bone tissue engineering. Part I: morphological, physical, and mechanical characterization, International Journal of Nanomedicine, 93-100.

Blitterswijk C. (2008). Tissue Engineering. San Diego, CA, USA: Elsevier.

Bondeson D., Mathew A., and Oksman K. (2006). Optimization of the isolation of nanocrystals from microcrystalline cellulose by acid hydrolysis. Cellulose, 13, 171-180.

Buyanov A.L, Gofman I.V, Revel'skaya L.G, Khripunov A.K, Tkachenko A. (2010). Anisotropic swelling and mechanical behaviour of composite bacterial cellulosepoly(acrylamide or acrylamide-sodium acrylate) hydrogels. Journal of mechanical behaviour of biomedical materials, 3, 102-111.

Callister W. (2007). An Introduction to Composites. New York, PA, USA: John Wiley \& Sons Inc.

Canal C, Ginebra M. P. (2011). Fiber-reinforced calcium phosphate cements: A review. Journal of the mechanical behaviour of biomedical materials, 4, 1658-1671.

Chu P.K. and Xuanyong L. (2008) Biomaterials Fabrication and Processing Handbook, CRC Press, Taylor \& Francis, Chapter 5, page 132.

Ciobanu G., Ignat D., and Luca C., (2009). Polyurethane-hydroxy apatite bionanocomposites: Development and characterization. Journal of Chemistry Bulletin, $54,57-68$.

Davies J. (1980). Mechanisms of endosseous integration. International of Prosthodont, $11(5), 391-401$.

Eftekhari S., \& Bougherara H. (2014). Patent No. 61/941,492. United States of America. 
Eftekhari S., El Sawi I., Bagheri Z., Turcotte G., \& Bougherara, H. (2014). Fabrication and characterization of novel biomimetic PLLA/cellulose/hydroxyapatite nanocomposite for bone repair application. Materials Science and Engineering C, 39, 120-125.

Einchhorn S.J., Dufresne A., Aranguren M., Marcovich N.E., Capadona J.R., Rowan S.J., Weder C., and et al. (2010). Review: Current international research into cellulose nanofibers and nanocomposites. Journal of Materials Science 45, 1-33.

Espert Ana, Vilapana F., Karlsson S. (2004). Comparison of water absorption in natural cellulosic fibers from wood and one-year crop in polypropylene composites and its influence on their mechanical properties. Composites: Part A, 35, 1267-1276.

Felfel R.M., A. I., Ahmed I., Parsons A.J., Rudd C.D. (2013). Bioresorbable screws reinforced with glass fiber: Manufacturing and mechanical property characterization. Journal of mechanical behavior of biomedical materials, 17, 76-88.

Flahaut E. (2005). Catalytic CVD synthesis of double and triple-walled carbon nanotubes by control of the catalyst preparation. Carbon, 4, 375-383.

Frone A. (2011). Some aspects concerning the isolation of cellulose micro- and nanofibers. U.P.B. Science Bulletin, 73 (2), 133-152.

Gay S., A. (2009). Preparation and characterization of dense nanohydroxyapatite/PLLA composites. Materials Science and Engineering C, 29, 172-177.

Gning P.B., L. S. (2011). Influence of process and test parameters on the mechanical properties of flax/epoxy composites using response surface methodology. Journal of Materials Science, 46, 6801-6811.

Goetz L., \& Oksman, K. (2009). A novel nanocomposite film prepared from crosslinked cellulosic whiskers. Carbohydrated Polymers, 75, 85-89.

Graupner N., H. A. (2009). Natural and man-made cellulose fiber-reinforced poly (lactic acid) (PLA) composites: An overview about mechanical characteristics and application area. Composite Part A-Applied Science Manufacturing, 40 (6-7), 810-821.

Hilding J., \& Lockwood, F. (2003). Dispersion of Carbon Nanotubes in Liquids. Journal of Dispersion Science and Technology, 24, 1-41.

Hsu Y.Y., Gresser J.D., Trantolo D.J., Lyons C.M., Gangadharam P.R., Wise D.L. (1997). Effect of polymer foam morphology and density on kinetics of in vitro controlled release of isoniazid from compressed foam matrices. Journal of Biomedical Material Research, 35, 107-116.

J. G. Williams, Stress analysis of polymers, Halsted press, New York, 1973. pp. 275 
Joulazadeh M, and Navarchian A. H. (2010). Effect of process variables on mechanical properties of polyurethane/clay nanocomposites. Polymers Advanced Technologies, 21, 263-271.

ISO,10993-5. (2009). Biological evaluation of medical devices, Part 5: Tests for in vitro cytotoxicity. International Standard. ISO.

Kanigan R. (2006). Market evaluation of demineralized bone matrix products in Canada: Research highlights. Peak Research, Inc. Canadian Council for Donation and Transplantation.

Kim H.W., Lee H-H, and Chin G-S. (2008). Bioactive and osteoblast responses of novel biomedical nanocomposites of bioactive glass nanofiber filled poly (lactic acid). Journal of Biomedical Materials Research: Part A, 85, 651-663.

Kong Y, Hay J.N. (2002). The measurement of the crystallinity of polymers by DSC. Polymer, 43, 3873-3878.

Lewtus D., McCarthy S., Ophir A., Kenig S. (2006). The effect of nanoclays on the properties of PLLA-modified polymers part 1: Mechanical and thermal properties. Journal of Polymers and Environments, 14, 171-177.

Bo Li, Feng-Xia Dong, Xiu-Li Wang, Juan Yang, De-Yi Wang, Yu-Zhong Wang. (2009). Organically modified rectorite toughened poly (lactic acid): Nanostructures, crystallization, and mechanical properties. European Polymer Journal, 45, 2996-3003.

Guan L. (2003). MASc. Thesis. A highly macroporous biodegradable composite scaffold for bone tissue engineering application. Toronto, Canada: University of Toronto.

Liu D., and Easteal, A. (2010). Characterization of solution cast cellulose nanofiberreinforced poly (lactic acid). eXPRESS Polymer Letters, 4, 26-31.

Ma P., and Zhang, R. (2002). Protein-mediated osteoblast survival and migration on biodegradable polymer/hydroxyapatite composite scaffolds. Transaction of the 28th annual meeting of society for biomaterials, 605 .

Majdzadeh-Ardakani K. N. (2010). Optimization of mechanical properties of thermoplastic starch/clay nanocomposites. Carbohydrated Polymers, 79, 547-554.

Martens M., Audekercket V., Delport P., Meester P.D., and Mulier J.C. (1998). The mechanical characteristics of the cancellous bone at the upper femoral region. Journal of Biomechanics, 16, 12, 971-983.

Mattila R. (2004). Production and structural characterization of porous fiber-reinforced composites. Composites, Part A, 35A, 631-636. 
McCulloch J. (2009). Regenerative Medicine, industry briefing, commercial opportunities and Ontario's strength. MaRS advisory service.

Medipoint. (2014). Bone grafts and substitutes-US analysis and market forecasts. GlobalData.

Morgan R., and Ramakrishna S. (2005). Development of nanocomposites for bone grafting. Composite Sience Technology, 65, 2385-2406.

Nejati E., Zandi, M., and Mirzadeh, H. (2008). Synthesis and characterization of nanohydroxyapatite rods/polyn (L-lactide acid) composite scaffolds for bone tissue engineering. Composites:Part A , 39, 1589-1596

Erika Nieddu, L. Mazzucco, P. Gentile, T. Benko, V. Balbo, R. Mandrile, G. Ciardelli (2009). Preparation and biodegradation of clay composites of PLA. Reactive \& Functional Polymers, 69, 371-379.

Okomoto Masami, J. B. (2013). Synthetic biopolymer nanocomposites for tissue engineering. Progress in polymer science, 38, 1487-1503.

Pei A., Zhou Q., Berglund L.A. (2010). Functionalized cellulose nanocrystals as biobased nucleation agents in poly(L-lactide) (PLLA)-Crystallization and mechanical property effects. Composites Science and Technology, 70, 815-821.

Román A. Pérez, Jong-Eun Won, Jonathan C. Knowles, Hae-Won Kim. (2013). Naturally and synthetic smart composite biomaterials for tissue regeneration. Advanced Drug Delivery Reviews, 65, 471-496.

Pooyan P., Tannenbaum R., and Garmestani H. (2012). Mechanical behaviour of a cellulose-reinforced scaffold in vascular tissue engineering. Journal of mechanical behavior of biomedical materials, 7, 50-59.

Ratner B.D., Hoffman, A., Schoen F.J., and Lemons, J.E. (2004). Biomaterials Science: An Introduction to Materials Medicine. San Diego, California, USA: Elsevier.

Reilly DT., Burstein A.H. (1974). The mechanical properties of cortical bone. Bone Joint Surgery, 56 (5), 1001-1022.

Rhim J.W., Hong S., Ha C.S. (2009). Tensile, water vapor barrier and antimicrobial properties of PLA/nanoclay composite films. Food Science and Technology, 42, 612-617. 
Roether J.A., Boccaccini A., Hench L.L. et al. (2002). Development and in vitro characterization of novel bioresorbable and bioactive composite materials based on polylactide foams and bioglass for tissue engineering applications. Biomaterials, 23, 3871-3878.

Sahithi K., and Selvamurugan, N. (2010). Polymeric composites containing carbon nanotubes for bone tissue engineering. International journal of biological macromolecules, 46, 281-283.

Bernard A. G. Schrauwen, Roel P. M. Janssen, Leon E. Govaert, and Han E. H. Meijer Dutch (2004). Intrinsic Deformation Behavior of Semicrystalline Polymers. Macromolecules, 37, 6069-6078.

M. Özgür Seydibeyoğlu, Kristiina Oksman. (2008). Novel nanocomposites based on polyurethane and microfibrillated cellulose. Composite Science and Technology, 68, 3-4.

Sturcova A., Davies G.R. and Stephen J.E. (2005). Elastic modulus and stress transfer properties of tunicate cellulose whiskers. Biomacromolecules, 6, 1055-1061.

Tesma Y., Raghavan D., and Stubbs J. (2004). Bone cell viability on collagen immobilized poly (3-hydroxy butrate-co-3-hydroxyvalerate) membrane: Effect of surface chemistry. Applied polymer Science, 93, 2445-2453.

Voet D. (1995). Biochemistry. John Wiley \& Sons, Inc.

Wang L. (2009). Porous bioactive scaffold of aliphatic polyurethane and hydroxy apatite for tissue regeneration. Journal of Biomedical Research, 4, 1-7.

Ward I.M. and Sweeney J. (2004). An Introduction to the Mechanical Properties of Solid Polymers (Second ed.). Chichester, England: John Wiley \& Sons, Ltd.

Wei, G., and Ma, P. (2004). Structure and properties of nano-hydroxyapatite/polymer composite scaffolds for bone tissue engineering. Biomaterials, 25, 4749-4757.

Wolfgang G., Seidler S. (2007). Polymer Testing. Vienna, Austria: Hanser Gardner Publication, Inc.

Xiao Y., Li D., Fan, H., Li, X., Gu Z., Zhang, X. (2007). Preparation of nano-HA/PLA composite by modified-PLA for controlling the growth of HA crystals. Material letters, $61,59-62$.

Xie X.L., and Zhou, X.P. (2005). Dispersion and alignment of carbon nanotubes in polymer matrix: A review. Materials Science and Engineering, 49, 89-112. 
Yang L., and Kandel, R. (2009). Polar surface chemistry of nanofibrous polyurethane scaffold affect annulus fibrousus cell attachment and early matrix accumulation. Biomedical Material Research, $91 \mathrm{~A}$.

Yang, S. and Leong, K. (2001). The design of scaffolds for use in tissue engineering. Tissue Engineering, 76, 679-689.

Kai Y., Hamada J.-I., Morioka M., Tokada T., Hasegana S., Ushio Y. (2000). The utility of the microcrystaliine cellulose sphere as a particulate embolic agent: An experiment study. Neuroradiol, 21, 1160-1163.

Zadegan S., Hosainalipour M., Rezaie H.R., Ghassai H., Shokrgozar M.A., Materials Science and Engineering: C.2011, 31, 954.

Zerda Adam.S. and Lesser, A.J. (2001). Intercalated Clay Nanocomposites: Morphology, Mechanics, and Fracture Behaviour. Journal of Polymer Science: Part B: Polymer Physics, 39, 1137-1146.

Zhang R.Y. and Ma. P.X. (1999). Poly (alpha-hydroxyl acids)/hydroxyapatite porous composites for bone-tissue engineering. I. Preparation and morphology. Journal of Biomedical Material Research, 44, 446-455.

Zimmermann K.A., LeBlanc J.M., Sheets K.T., Fox R.W., Gatenholm P. (2011) Biomimetic design of a bacterial cellulose/hydroxyapatite nanocomposite for bone healing applications, Materials Science and Engineering: C.31, 43-49. 